\title{
Green Wireless Cellular Networks with Renewable Energy Resources \\ Using Stream Control Transmission Protocol (SCTP)
}

\author{
by \\ Abdullah Aljanah \\ A thesis submitted to the \\ Faculty of Graduate and Postdoctoral Studies \\ In partial fulfilment of the requirements \\ For the M.A.Sc. degree in Electrical and Computer Engineering \\ Ottawa-Carleton Institute for Electrical and Computer Engineering \\ Carleton School of Information Technology \\ Carleton University
}

(C)Abdullah Al-Janah, Ottawa, Canada, 2012 
Library and Archives

Canada

Published Heritage

Branch

395 Wellington Street

Ottawa ON K1A ON4

Canada
Bibliothèque et

Archives Canada

Direction du

Patrimoine de l'édition

395 , rue Wellington

Ottawa ON K1A ON4

Canada
Your file Votre référence

ISBN: 978-0-494-94256-7

Our file Notre référence

ISBN: 978-0-494-94256-7
NOTICE:

The author has granted a nonexclusive license allowing Library and Archives Canada to reproduce, publish, archive, preserve, conserve, communicate to the public by telecommunication or on the Internet, loan, distrbute and sell theses worldwide, for commercial or noncommercial purposes, in microform, paper, electronic and/or any other formats.

The author retains copyright ownership and moral rights in this thesis. Neither the thesis nor substantial extracts from it may be printed or otherwise reproduced without the author's permission.
AVIS:

L'auteur a accordé une licence non exclusive permettant à la Bibliothèque et Archives Canada de reproduire, publier, archiver, sauvegarder, conserver, transmettre au public par télécommunication ou par l'Internet, prêter, distribuer et vendre des thèses partout dans le monde, à des fins commerciales ou autres, sur support microforme, papier, électronique et/ou autres formats.

L'auteur conserve la propriété du droit d'auteur et des droits moraux qui protege cette thèse. $\mathrm{Ni}$ la thèse ni des extraits substantiels de celle-ci ne doivent être imprimés ou autrement reproduits sans son autorisation.
In compliance with the Canadian Privacy Act some supporting forms may have been removed from this thesis.

While these forms may be included in the document page count, their removal does not represent any loss of content from the thesis.
Conformément à la loi canadienne sur la protection de la vie privée, quelques formulaires secondaires ont été enlevés de cette thèse.

Bien que ces formulaires aient inclus dans la pagination, il n'y aura aucun contenu manquant. 
The undersigned recommend to

the Faculty of Graduate and Postdoctoral Affairs

acceptance of the thesis

\title{
Green Wireless Cellular Networks with Renewable Energy Resources Using Stream Control Transmission Protocol (SCTP)
}

\author{
Submitted by \\ Abdullah Aljanah, B.Sc., M.Eng. \\ In partial fulfillment of the requirements for the degree of \\ Master of Applied Science in Electrical and Computer Engineering
}

Chair, Professor Howard Schwartz,

Department of System and Computer Engineering

Thesis Supervisor, Professor Richard Yu

Carleton University

November 2012 


\section{Abstract}

Renewable energy and green technologies are important global topics that are expected to affect the way of life of future generations. Deploying a green wireless principle will help to reduce the world's dependency on fossil fuels, consequently lowering carbon dioxide and other emissions as will. In this thesis, we propose a new approach to employ the Stream Control Transmission Protocol (SCTP) in a heterogeneous network of wired and wireless/mobile nodes while maintaining quality and reliability of service availability when switching power sources. This switching between sources exists in situations where a renewable energy (e.g., solar or wind) fed small cell base station (green cellular) is used as an alternative and assistant to a traditional energy fed base station. Since renewable energy sources may not be reliable at all times, the proposed system employs SCTP to make the handover between a base station fed by renewable energy source and another base station fed by conventional energy source. If the renewable energy source becomes unreliable for even a few seconds, the system is switched over to the traditional base station fed by conventional energy source, until the renewable energy fed base station is once again reliable. Simulation results are presented to show that SCTP can manage the handover between the conventional energy fed base stations and renewable energy fed base stations smoothly, even when renewable energy sources are not stable and the energy level fluctuates. 


\section{Acknowledgements}

I'd like to give my deepest thanks and gratitude to my supervisor Professor Richard $\mathrm{Yu}$, under whose supervision I had the honour of conducting my research.

- Abdullah Aljanah

August 2012 


\section{Table of Contents}

Abstract $\quad$ ii

$\begin{array}{ll}\text { Acknowledgements } & \text { iii }\end{array}$

List of Tables vii

List of Figures viii

1 Introduction 1

1.1 Research Overview and Background . . . . . . . . . . . . . 1

1.2 Motivation and Objectives . . . . . . . . . . . . 5

1.3 Contributions ........................ 7

1.3.1 Submitted/Accepted Papers . . . . . . . . . . . . 8

1.4 Thesis Organization . . . . . . . . . . . . . . . . . . 9

2 Literature Review and Related Works 11

2.1 Green Wireless Technologies Overview . . . . . . . . . . . 11

2.2 Renewable Energy Resources . . . . . . . . . . . . . . . . . . . 13

2.3 Transport Layer Overview . . . . . . . . . . . . . . . 18

2.3 .1 SCTP Overview . . . . . . . . . . . . 20

2.4 SCTP vs TCP vs UDP . . . . . . . . . . . . . . 21 
2.5 Related Work . . . . . . . . . . . . . . . . . . . 23

2.5.1 Improving TCP Performance During Intra LTE Handover . . 25

2.5.2 Analysis of Real-time Multimedia Transmission over PR-SCTP 26

2.5.3 SCTP Efficient Flow Control During Handover . . . . . . . 27

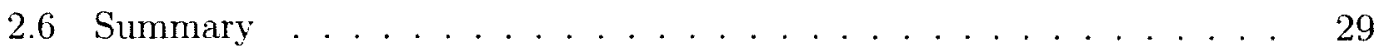

3 Improved Green Cellular Techniques by Employing SCTP Based $\begin{array}{ll}\text { Handover Mechanism } & 30\end{array}$

3.1 Network Topology . . . . . . . . . . . . . . . . . 30

3.1 .1 Outdoor Small Cells . . . . . . . . . . . . . . . . . 31

3.2 Heterogeneous Hierarchical Topology Addressing . . . . . . . . . 33

3.3 Simulation Environment and LTE Models . . . . . . . . . . . . 34

3.4 TCP and SCTP Agents and the Hierarchical Addressing $\ldots \ldots \ldots 37$

3.4 .1 Handover Scenario and Traffic . . . . . . . . . . . 38

3.5 Summary . . . . . . . . . . . . . . . . . . . 40

4 Simulation Results and Discussions $\quad 41$

4.1 Network Simulation Tools . . . . . . . . . . . . . . . . . . 41

4.1.1 Simulation Tools Survey and Comparison . . . . . . . . . . 43

4.1 .2 NS-2 Simulation Tool . . . . . . . . . . . . . . . 44

4.2 Results of TCP and SCTP based Handovers . . . . . . . . . 49

$4.2 .1 \quad$ FTP Traffic . . . . . . . . . . . . . . . . . . 49

4.2 .2 Audio Traffic . . . . . . . . . . . . . . . . 52

4.2 .3 Video Traffic . . . . . . . . . . . . . . . . . 53

4.3 Summary $\ldots \ldots \ldots \ldots \ldots \ldots \ldots \ldots \ldots \ldots \ldots$

5 Conclusions and Future Work $\quad 61$

5.1 Conclusions . . . . . . . . . . . . . . . . . 61 
5.2 Future Work . . . . . . . . . . . . . . . . . . . . 63

References

A Simulation Scripts

74

A.1 FTP and Audio Traffic via TCP and SCTP . . . . . . . . 74

A.2 Video Traffic via TCP and SCTP . . . . . . . . . . . . . . 90 


\section{List of Tables}

2.1 A comparison of SCTP, TCP and UDP . . . . . . . . . 24

4.1 Key Differences between Simulation Tools . . . . . . . . . . . . . . 43

4.2 Trace File Format for TCP . . . . . . . . . . . . . . . . . . . . 48

4.3 Trace File Format of TCP for the Wireless Part . . . . . . . . . . . . 48

4.4 Trace File Format for SCTP . . . . . . . . . . . . . . . . . . . . 49

4.5 Trace File Format of SCTP for the Wireless Part . . . . . . . . . . . 49 


\section{List of Figures}

1.1 Energy consumption in a mobile network [6] . . . . . . . . . 4

1.2 Power saving dimensions in mobile network [8] . . . . . . . . 6

2.1 Heterogeneous network $[11] \ldots \ldots \ldots$

2.2 Geothermal processes $[18] \ldots \ldots \ldots \ldots$

2.3 Geothermal ORC system $[19] \ldots \ldots \ldots$

2.4 Drawing of the rotor and blades of a wind turbine $[19] \ldots \ldots$

2.5 Power generated for several sizes of turbines $[19] \ldots \ldots$. . . . . . . . 19

2.6 Photovoltaic array interface $[8] \ldots \ldots \ldots$

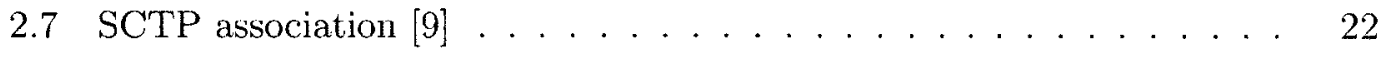

2.8 SCTP packet format $[9] \ldots \ldots \ldots \ldots \ldots \ldots$

2.9 Solar and wind combined renewable energy for a cellular network [36] 28

3.1 Wired-cum-wireless heterogeneous topology . . . . . . . . . . . 31

3.2 Wired-cum-wireless heterogeneous topology clouds . . . . . . . . 32

3.3 Assumption of simi-straight motion with respect to a small cell . . . 33

3.4 Hierarchical addresses of the network elements . . . . . . . . . 35

3.5 The handover procedure . . . . . . . . . . . . . . 36

3.6 YUV video sequence-352x288 Resolution . . . . . . . . . . . . 37

4.1 Basic architecture of $n s-2[53] \ldots \ldots \ldots \ldots . \ldots . \ldots 45$ 
4.2 SCTP vs. TCP TSN behaviour for FTP traffic . . . . . . . . . 50

4.3 SCTP vs. TCP instantaneous throughput behaviour for FTP traffic 51

4.4 SCTP vs. TCP delay behaviour for FTP traffic . . . . . . . . . 52

4.5 SCTP vs. TCP TSN behaviour for audio traffic . . . . . . . . . 54

4.6 SCTP vs. TCP instantaneous throughput behaviour for audio traffic $\quad 55$

4.7 SCTP vs. TCP delay behaviour for audio traffic $\ldots \ldots . \ldots 56$

4.8 SCTP vs. TCP TSN behaviour for video traffic . . . . . . . . . 57

4.9 SCTP vs. TCP instantaneous throughput behaviour for video traffic 58

4.10 SCTP vs. TCP delay behaviour for video traffic . . . . . . . . . 59

4.11 Average delay of TCP and SCTP for the simulated traffic types . . . 60 


\section{Chapter 1}

\section{Introduction}

\subsection{Research Overview and Background}

Over the last three decades, global warming has become a very important topic, and the use of renewable energy is widely considered a key factor in dealing with the issue. Since the beginning of the Industrial Revolution in the second half of the eighteenth century, more than one trillion tons of fossil fuels have needed to be burned in order to obtain the amount of energy required to meet humanity's increasing demands. This has mainly been because of the energy requirements from the growing number of factories all over the world [1]. In fact, regardless of the traditional uses of the pre-Industrial Revolution period resources, such as the wood for cooking and heating, 400 trillion tons of carbon dioxide have been launched to the Earth's atmosphere since 1850. For example, in a report by the U.S. Energy Information Administration (EIA), it is stated that electricity generation was responsible for the emission of 2,359 million metric tons of carbon dioxide in $2008[2,3]$. The consequences of these increasing carbon emissions have been global warming and climate change. This has resulted in a significant negative impact on ecosystems and the lives of all of the Earths creatures. 
There are several studies claiming that global warming is not the result of carbon dioxide and that it is due to some other factors such as the solar activity period (solar spots and solar magnetic field variation). In addition, some research points the finger at the axis of the Earth's slope fluctuating. These researchers believe that the world is in a warm period between ice ages (little ice ages), however, it is a certain and indisputable fact that increasing carbon emissions directly affects the Earth's atmosphere and leads to a dramatic disturbance in environmental systems. This is more than enough of a reason to be concerned [2].

Due to the aforementioned statistics, people have responded positively in terms of combating global warming through science and technology. Since carbon dioxide (and the other types of gases that contribute to global warming) are emitted in the energy production process in the industrial realm (mainly in electric power generation), one solution for mitigating the effect of global warming is to evolve technologies that use less of this energy. It would be better to operate and feed our modern activities with clean energy sources instead of dirtier, traditionally-produced energy. The less carbon emissions, the more stable the climate will be.

Clean energy initiatives are referred to as green technologies in the field of information and communication technologies (ICT). More specifically, "green cellular networks" refer to mobile networks where the communication links (mainly wireless) and the network elements (mobile base stations, switches, network core cabinets, etc.) are treated, reconstructed, and redesigned to be environmentally friendly.

Mobile cellular networks are an obvious player in this contemporary age of communications. From the consumer's point of view, mobile cellular networks have achieved a complete personality and independence for the first time in history. As anticipated by Gartner, by 2013 mobile phones will be the dominant web access device (more than $\mathrm{PCs})$. In fact, the total number of PCs is expected to be 1.78 billion units, while the 
number of well enhanced mobile phones will exceed 1.82 billion units. Gartner also states that by 2014 more than 3 billion people will be generating transactions via mobile or Internet technology, and there will be around 1.8 billion mobile units [4]. As a result, more energy will be required to feed these rapidly growing mobile cellular networks.

Because the frequency band is a limited network resource, frequency reuse has been the most significant and efficient factor for mobile cellular network technologies to succeed. From a technical point of view, the more users that are served by the frequency band means the more frequency reuse that is needed. However, more frequency reuse means more cells need to be established, designed, constructed, and fed by electrical power. As the number of cells increases, the number of base stations (BSs) will also increase to cover more users [5]. By analyzing the power budget and power consumption within mobile networks, especially cellular mobile networks, it has been found that the most power consumption and dissipation is in the mobile BSs, regardless of the type of the cellular system (GSM, 2.5G, 3G, etc.) or the range of the wireless cell (macrocell, microcell, picocell or small cell). An example is shown in Figure 1.1.

There are several dimensions that designers and regulators go through in order to obtain and satisfy the trend of green technologies in BSs. These dimensions are shown in Figure 1.2 and can be summarized as follows:

- The first dimension is to reduce the BS energy consumption (energy saving). One example is the minimization of the energy consumption of power amplifiers (PAs) in the BS, which are required for radio communication (transmitters and receivers). PA's consume from $50 \%$ to $80 \%$ of the BS energy. Also, BS energy savings can be obtained by applying a power saving protocol in the media access control (MAC) layer by applying energy-aware mechanisms (e.g. cell zooming), 


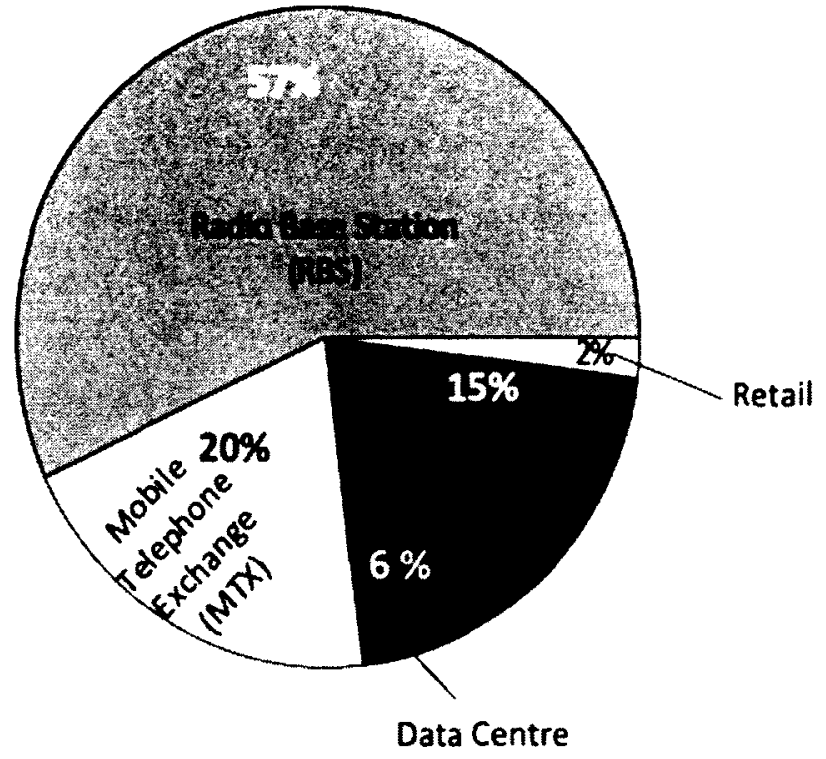

Figure 1.1: Energy consumption in a mobile network [6]

by using a direct sustainable energy resource (e.g. solar, wind, or biofuel), or by applying energy aware management.

- The second dimension is to reconfigure the network plan and go forward to heterogeneous network, or in other words, move from macrocells to micro or small cell for more power efficient BSs.

- The third dimension is to use green wireless technologies (at the physical layer) to encourage more spectrum utilization. This is seen in cognitive radio and technologies that add more improvement to throughput, such as cooperative relaying. 


\subsection{Motivation and Objectives}

Since renewable energy techniques do no emit global warming gases, like carbon dioxide, sulphur dioxide, nitrogen dioxide, etc., the more they are implemented. the more the world and environment will be protected from climate change because it will help to reduce the greenhouse effect. In addition to the global warming effect, these gases are also directly responsible for the acid rain phenomenon. This happens when these gases reacts with water molecules in the atmosphere and became micro drops of acid solution. These come down as rain and cause negative effects on forests and water systems, and consequently, have a negative impact on ecosystems as a whole.

There are also other dimensions of global concern in using conventional energy. In many cases the natural resources industries are negatively affected when fossil fuel use increases while the national population continues to grow, especially in the oil producing countries. The problem is not so much related to pollution and acid rain because there are other dimensions that are considered more important using the conventional energy alone. It is the water resources dissipation due to the usage of fossil fuel in power generation. Water is used intensively for cooling in fossil fuel power station while the renewable power generation requires only very little amount of water [7].

In some cases, a huge part of national oil production will go to local usage instead of to exports (ALWATAN newspaper, date:30th of Jan 2012). For example, the Kingdom of Saudi Arabia consumes more than $10 \%$ of its own oil production. The daily consumption is approximately 2.4 million barrels. A remarkable portion of this daily oil consumption is reserved for electric power generation. Now consider that a large portion of Saudi Arabia is covered by desert, and solar energy would be readily available (the Arabian Peninsula is located in a band of desert). In addition, wind activity from the north to south is periodic, which provides a good alternative for 


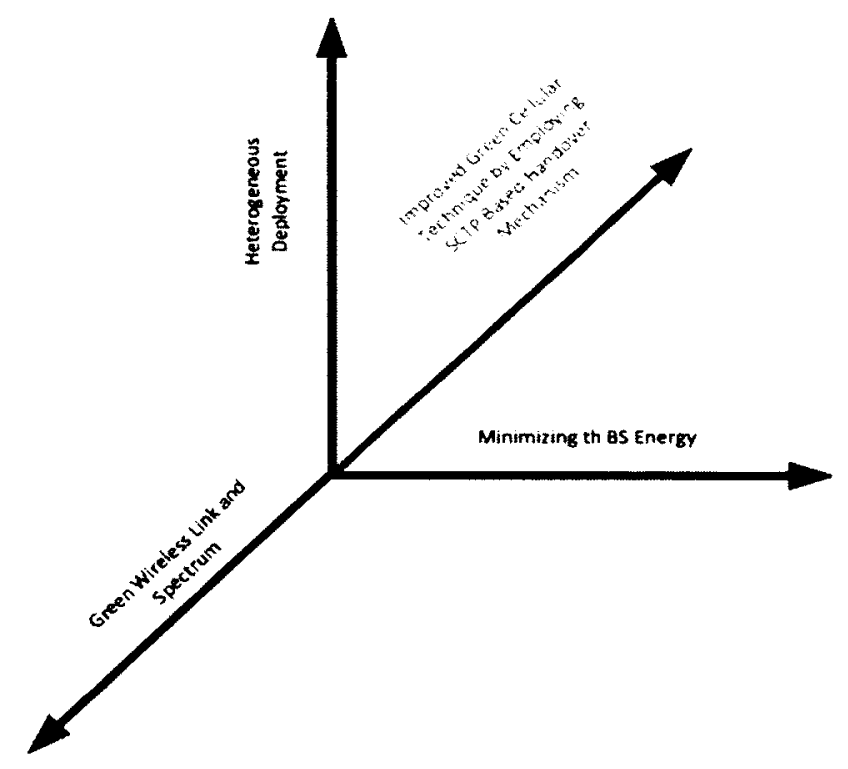

Figure 1.2: Power saving dimensions in mobile network [8]

reducing the dependency on oil for electric power. This is especially true for rural areas and for self-managed campuses such as business compounds.

Consider the Saudi Telecom Company (STC), whose mobile base stations are fed by conventional power supplies. It costs more than 100 million Saudi Riyals (one US dollar equals 3.75 Saudi Riyals) for the company to pay the electricity invoice for one year. Thus, using the green cellular concept (especially solar and wind energy) can benefit telecom service providers by helping them to manage power consumption and give them a competitive edge while keeping in mind that:

- Use of green cellular by both the service provider and end user is highly recommended. This scenario happens when the end user has some part of the ownership of the network elements that connect the private user to the network backbone (such as private branch exchange). The private, outdoor small cell access point can be managed by installing green cellular access points using renewable energy. 
- Some questions may arise regarding why the Telecom providers do not switch all of their access points or BSs to renewable energy sources. The answer is that this migration scenario is not attractive because the initial cost (CAPEX) to do so is very high, and none of the decision makers, such as bankers or investors, will support this, especially in a global financial crisis.

- Once there is a suitable protocol to support the quality of service and reliability of using green cellular techniques, stakeholders and decision makers will both invest in adopting the technology and enhancing the performance. Furthermore, they will positively affect political views and decisions on new environmental policies.

\subsection{Contributions}

This thesis proposes a new approach to employing the Stream Control Transmission Protocol (SCTP) in a heterogeneous network of wired and wireless/mobile nodes while maintaining quality and reliability of service availability when switching power sources. SCTP is used to maintain the transport layer connection and workflow due to its advanced features when compared to current conventional protocols, such as TCP (Transmission Control Protocol) and UDP (User Datagram Protocol). SCTP can add value in applying renewable energy supplies in green mobile networks for outdoor small cell as an appropriate paradigm. Through simulations, it has been proven that SCTP can manage the handover between the conventional energy fed BSs and renewable energy BSs smoothly and safely, even when renewable energy sources are not stable and the energy level fluctuates and is unreliable. The performance and the behaviour of the SCTP-based handover satisfies the metric criteria, such as

time delay and throughput. In addition, it is also possible to partially apply green 
wireless as a first stage of migration, especially for end users, small businesses or even in rural/poor areas where the economic dimensions force people to look for an alternative power supply. This can be done using highly available renewable energy sources as a concurrent companion and support to BSs or access points that are fed by conventional energy supplies. Finally, several traffic streams, such as FTP (file transfer protocol) and audio and video, were used to show that SCTP successfully maintains the transport connection and manages the handover between the access towers (fed by conventional energy and by the renewable energy). Even when the handover between the two access points occurs several times, SCTP connections remain safe and conserved. SCTP have been used for maintaining the handover, but the purposes and the conditions is quite different. Unlike the other previous works, in this work the environment is true wireless and the users (especially the user to be handed over between the base station) are moving. Also the hand over purposes and scenario is employed to save the energy and use the renewable energy as much as possible because we consider a fluctuating renewable energy resource. Therefore, the handover is subjected to occur many times in a short period. In addition to that, the

other new thing is that the Long term evolution (LTE) environment is considered in this work simulation.

\subsubsection{Submitted/Accepted Papers}

- Majed Alowaidi, F. Richard Yu, Abdulmotaleb El Saddik, and Abdullah Aljanah, "Improving Performance of Smart Grid Communications Using Multihoming and Multi-streaming Offered by SCTP", in Proc. IEEE SmartGridComm2012, Tainan, Taiwan, Nov. 2012.

- Abdullah Aljanah, F. Richard Yu, Majed Alowaidi, and Abdulmotaleb El Saddik "Green Wireless Cellular Networks with Renewable Energy Resources Using 
Stream Control Transmission Protocol (SCTP)", submitted to IEEE ICC'2013, Sept. 2012.

- Abdullah Aljanah, F. Richard Yu, Majed Alowaidi, and Abdulmotaleb El Saddik "Green Wireless Cellular Networks with Renewable Energy Resources Using Stream Control Transmission Protocol (SCTP)", submitted to International Conference on Clean Electrical Power, Oct. 2012.

\subsection{Thesis Organization}

This thesis is organized as follows:

- Chapter 1 provides an overview of the thesis research that has been conducted. It mentions the motivations, objectives, and challenges for using SCTP as a protocol in green wireless networks.

- Chapter 2 provides informative background information and discusses related works. This includes the state-of-the-art technologies in the field of green mobile networks and how it has become the world trend. In addition, the main usable sources of renewable energy will be discussed. In terms of background information, a literature review about the transport layer in the TCP/IP networks will be discussed. Also, the new SCTP protocol will be introduced and discussed, described, and compared with TCP and UDP.

- Chapter 3 provides information about the proposed solution and evaluation techniques. The system topology and hierarchy will be proposed and explained. Also, the hierarchical addressing in the proposed wired-cum-wireless topology will be explained. The scenario that TCP and SCTP agents use to manage the handover process will be discussed. In addition the traffic streams types (FTP, 
audio and video) that have been used in the simulation will be pointed. Finally, the traffic generation and motion simulation are discussed as an essential part of our module.

- Chapter 4 covers the simulation results and behaviours. The simulation tool that has been employed and utilized with all needed patches and software modifications to jump the limitation of the simulation tool will be explored. Moreover, the simulation tools will be benchmarked and criticized according to the available tools in the field of network protocol simulation, showing the reasons for choosing our specific tool. Also we will demonstrate and describe the simulation output for several types of traffic in the case of admitting TCP and SCTP as transport protocols to assess their behaviour using the proposed evaluation metrics.

- Chapter 5 will summarize the current work and conclude the simulation results. In this chapter, it is shown that the proposed scenario satisfies all conditions and gives an acceptable metric value when compared to conventional transport protocols. Future work in this field is then proposed, as well as what could be pursued with regards to the SCTP protocol. 


\section{Chapter 2}

\section{Literature Review and Related}

\section{Works}

\subsection{Green Wireless Technologies Overview}

Mobile and wireless-based network operators and manufacturers have focused on meeting the rapid demands of the their customers, which entails fulfilling the needs of growing broadband services in their heterogeneous networks (Figure 2.1). As a result, energy costs have rapidly increased, which has in turn made people more aware of the effect of increasing energy consumption on the environment and economy. For example, a mobile network containing between 12000 and 15000 cellular sites with 2G and $3 \mathrm{G}$ technologies with 3 antennas (for 360 degree coverage) per site per technology, uses about 736000 megawatt-hours. This energy is enough to operate 68000 European family homes [10]. Not only is this a large amount of energy, but the carbon footprint of mobile networks is expected to increase year by year because there are more than four million base stations (BSs) all over the world. Economically, the operation costs of network BSs is even more expensive. The on-grid BS costs are around 3000 U.S. 
dollars a year. An off-grid BS. which is usually available in rural areas and fed by diesel engines, costs much more than that [11].

Addressing the issue of Green Communications has benefits to many stakeholders including those in industry, academic research, and government agencies. Green Communications allows the cellular industry to realize cost savings and lower their impact to the environment. Government agencies realize fulfillment of administrative goals for energy savings, as well as the development of standards and metrics. Researchers can push the boundaries of current technologies and theories in material science, distributed computing, and systems engineering. Research in this field also has great synergy with developments in "smart grid" applications, given the significant impact that cellular BSs maintain on power usage. Coordinated power control of cellular equipment and dynamic load balancing within the greater power grid can lead to transformational improvements in both communications and energy systems.

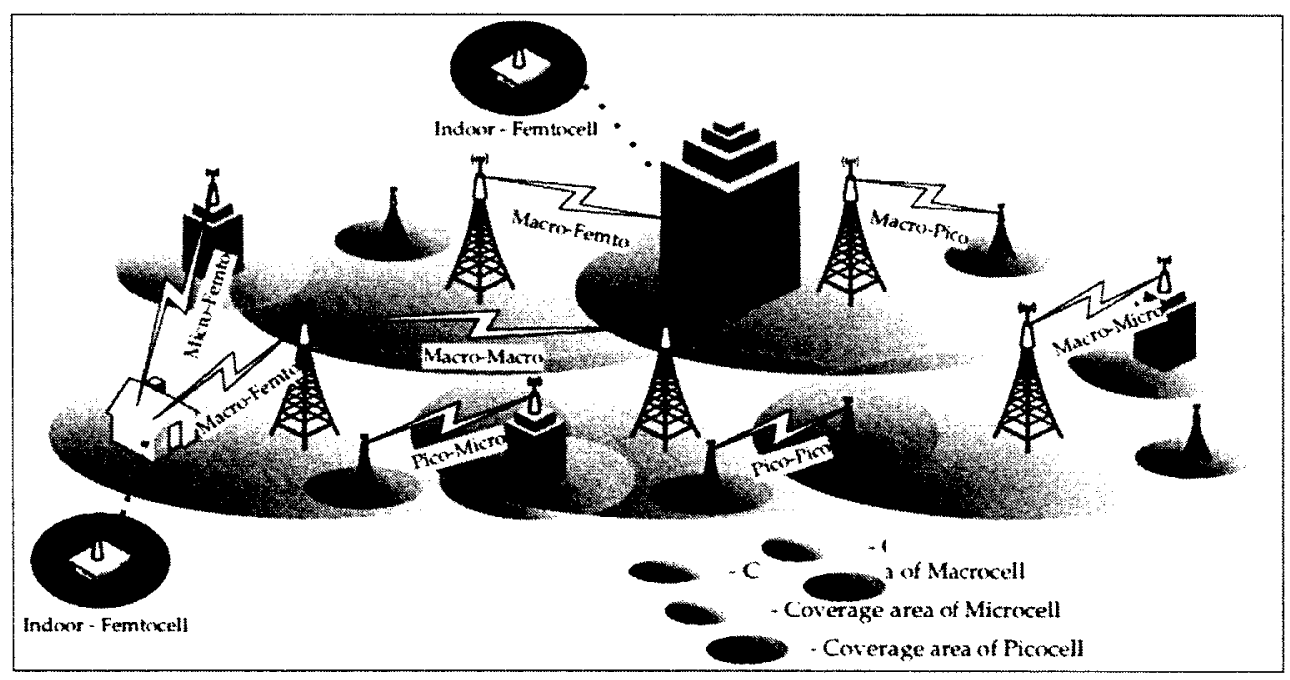

Figure 2.1: Heterogeneous network [11] 


\subsection{Renewable Energy Resources}

Renewable energies may be classified in terms of the source of the new energy being obtained as follows:

- Hydroelectric power is the most mature source of renewable energy, having been used since the nineteenth century. In this case, the renewable energy is obtained from the potential energy in the water flow. It takes on many forms due to the condition and position of the water source, however, it can be described in general as turbines being moved by the force of the water stream (mainly rivers). These turbines are connected to power generator rotors to produce the electricity in an alternative form. Man-made water dams are constructed to form a waterfall, which optimizes and maximizes the water's potential energy [12].

- Biomass alternative energy is also referred to as biofuels. Here the plant tissue (or in some cases materials derived from plant) are treated in a special way to produce energy, mainly in chemical form such as a gas or liquid. In addition to direct combustion to extract energy from the biomass (e.g. wood, planet residues, tree pulp, municipal wastes, etc.), there are several indirect processes to extract the biomass energy [13] [15]. Gasification, pyrolysis, digestion, and fermentation have been used to produce useful products in gas format (i.e. methane or hydrogen) or as a liquid formula (i.e. oil or alcohols). These products can be utilized to produce energy sources similar to the fossil fuel. This produced fuel is called biofuel; unfortunately, it is a carbon based fuel and it produces carbon dioxide. However, biofuel does not add more carbon dioxide to the atmospheric air, therefore it does not worsen global warming. This is because the original carbon in these materials came from the air when the plants 
grew (a very short period of time) and cannot be compared to the carbon that is inside the fossil fuel, which dates back to ancient geological ages such as the carbonic age (Carboniferous) [16] [14].

- Geothermal energy is another alternative that works especially well when local conditions are favorable. In these systems, the heat contained deep within the Earth can be utilized to heat water. This water is injected into pipes that penetrate towards the centre of the Earth. These pipes could reach several hundred meters in depth [17]. Water injected inside these pipes absorbs the extremely high ground heat (more than 240 degrees Celsius) [18] and is converted to steam. The steam is piped to a power plant where it passes through generator turbines before being condensed and injected back into the ground in a circulatory loop as shown in Figure 2.2. Geothermal energy is a non-depleting

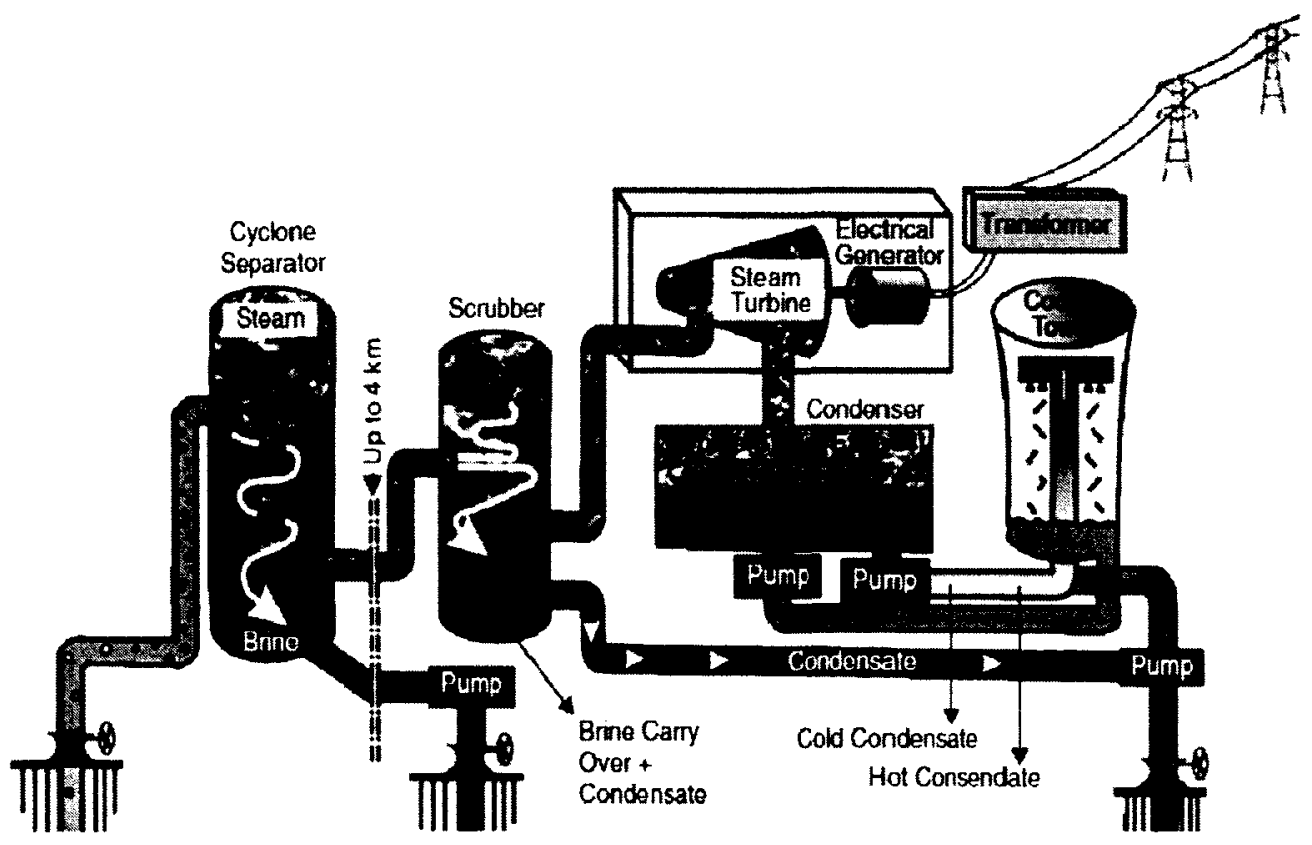

Figure 2.2: Geothermal processes [18] 
energy source (abundant), however, only a small amount of this type of energy can be converted to usable electric power [18]. In other words, using geothermal energy to produce electric power requires a very high temperature in order to evaporate the water, convert it to steam, and move it through the turbine. For example, if the geothermal water temperature is equal to or less than $100^{\circ}$ Celsius, the water can not be vaporized and the conventional turbine can not be used. In this case the Organic Rankine cycle (ORC) can be employed here using organic materials (e.g.Isopentan [19]). This organic material takes the heat transferred from the geothermal heat source and evaporates water at a lower temperature. The steam produced by the organic materials rotates in the turbines before the organic materials are liquefied in a condenser as shown in Figure 2.2. The organic materials are then cooled and pumped again to the heat exchanger in order to complete the cycle. The overall efficiency of the ORC system is $10 \%$ maximum, so a process called Kalina process is employed to get a higher efficiency. This involves the water being mixed with ammonia to replace the organic materials [19].

- For wind-based renewable energy, air currents are utilized to rotate turbines and generate electricity. This is a common technology today. There can be two or three blades powering the turbines. These wind turbines can be on a horizontal axis or a vertical axis from the Earths surface [18]. Wind power is a clean and impressive source of sustainable energy, especially under the condition of fluctuating prices of conventional energy. It has been applied in several countries. By 2009 some European Union $(E U)$ countries had achieved remarkable progress in using wind energy for electricity production. For example, Denmark had produced $19 \%$ of their total power from wind, Spain 11\%, Germany \%7. This technology has spread to other parts of the world, such as India and China [21] 


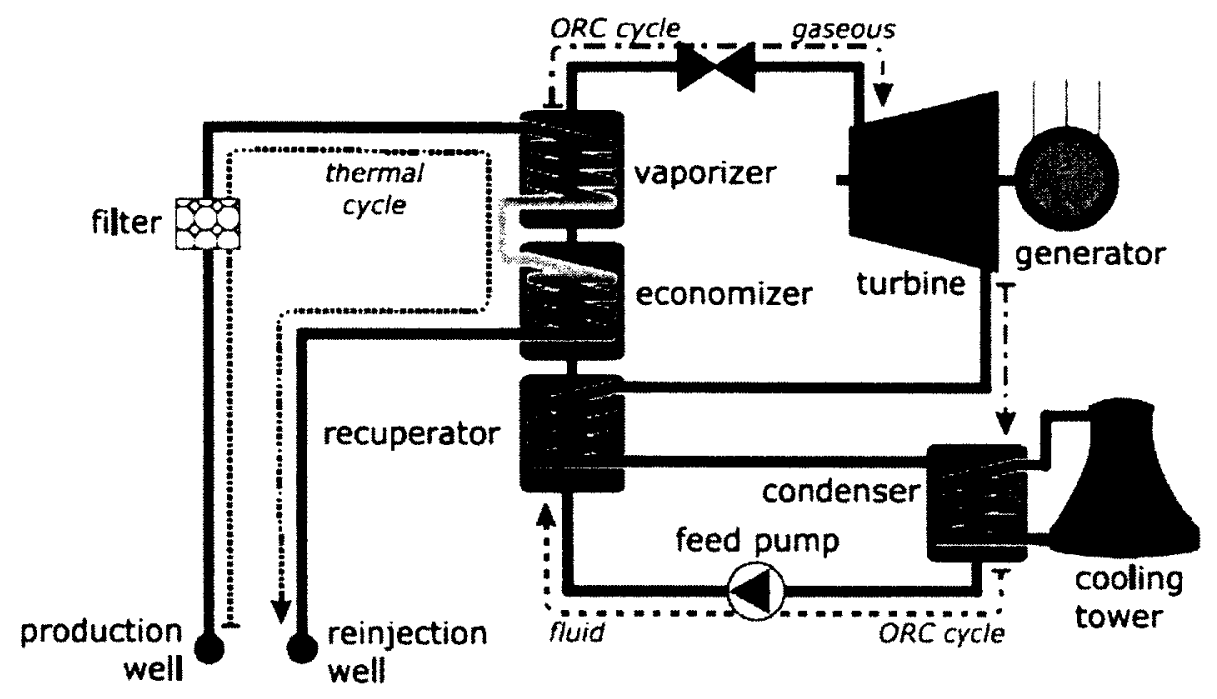

Figure 2.3: Geothermal ORC system [19]

in addition to the U.S. which is currently ranked third in global wind power usage $[23,24]$. From a theoretical point of view, the power obtained from the wind turbine is $P=(0.5) r h o A V^{3}[22]$ where: $\mathrm{P}$ is the power in watts, rho is the air density in $\mathrm{kg} / \mathrm{m}^{2}$, A is the swept area of the blade in $\mathrm{m}^{2}$ as shown in figure 2.4 and $V$ is the wind speed in $\mathrm{m} / \mathrm{s}$. Due to the practical conditions and material nature, the output of this equation needs to be multiplied with another parameter called the rotor power coefficient. This coefficient is a function of the blade pitch angle and the tip speed ratio, which means the output power is not constant. It is expected to fluctuate because the speed of the wind is timevariant due to the air density. Moreover, the transfer function of wind power to mechanical power in the wind turbine is non-linear, and the output power is zero when the wind speed is less than $3 \mathrm{~m} / \mathrm{s}$ !. The output power remains constant when the wind speed is over $15 \mathrm{~m} / \mathrm{s}$. It must, of course, be under the cut-off speed (for safety), which is around $25 \mathrm{~m} / \mathrm{s}$ [25], so that whenever 
the speed exceeds this limit the output power is shut off. This nature dilemma appears here as one of the most challenging for wind turbine renewable energy. To gain more power, the area factor (swept area) needs to be maximize as much as possible as a trade-off between the construction cost and the output power. As a practical example, Figure 2.5 demonstrates the variance in dimension for several wind turbine towers versus the obtained power magnitudes for each one. In rural or light residential areas, a single small wind turbine may be employed for power generation. However, to feed an industrial or urban district, especially when the wind impact varies, the wind turbines are grouped in large arrays (wind farms) and connected in a grid topology for higher utilization and synchronization $[23,26]$. It is essential to manage the communication control signals and data communication between the grid elements [26]. With these features, the main challenges in fields with wind power are the required land area, as well as to the time variation of the output power, which makes weather forecasting (wind data analysis and prediction) an essential factor in the wind power industry.

- Solar energy is one of the most common form of sustainable energy resources. This type of energy can be utilized directly as a heat spotted in a focus of a curved mirror or convex lens. This is a traditional way to collect sun energy for direct use, such as water boiling or cooking. The process of using large mirrors to concentrate the sun's heat energy to boil water and apply it to generate electric power is called solar thermal power [8]. Solar photovoltaic cells which are made from a photo-electric devices (semiconductors electronic) can produce some electric power due to the stimulation property of the semiconductor when exposed to light (electron photon energy exchanging). Thermal power (solar power) often is used for electric energy production because it is mature and 

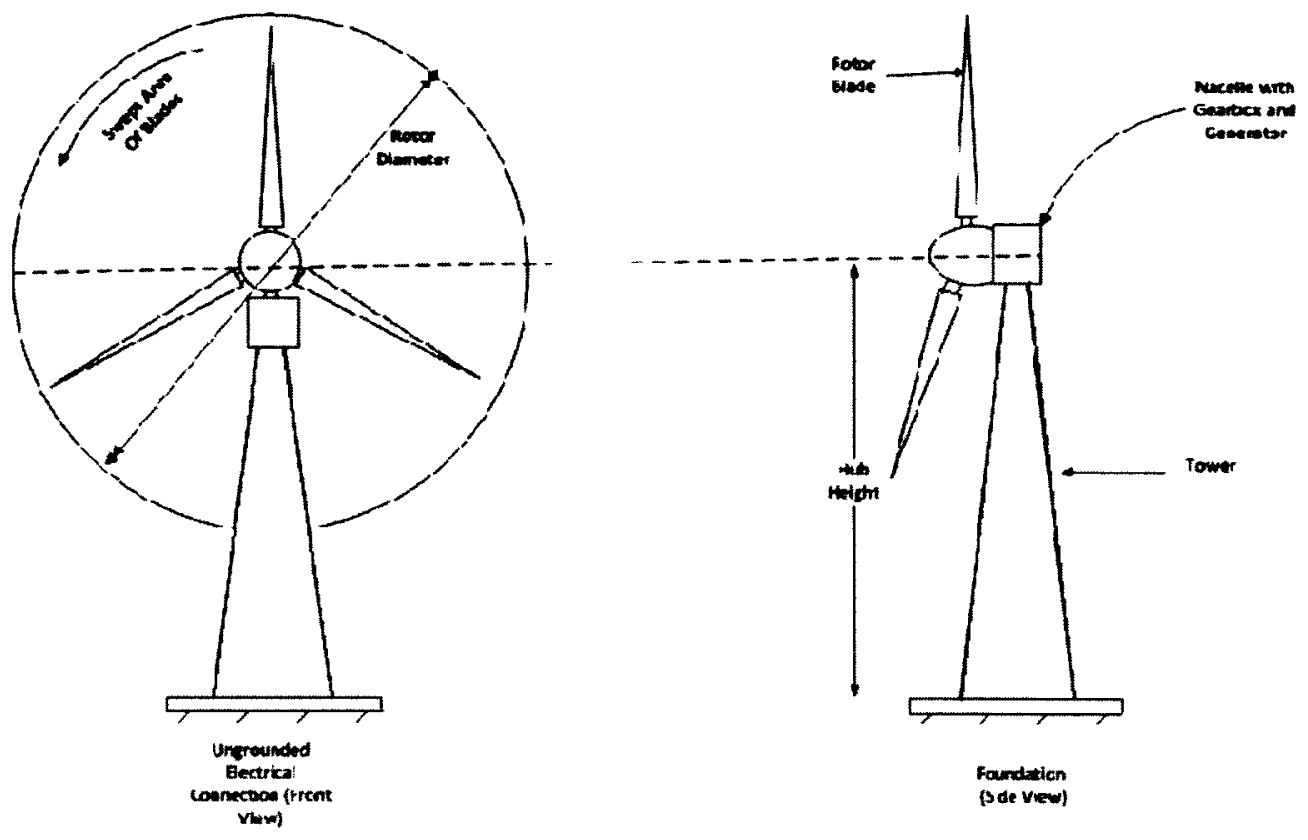

Figure 2.4: Drawing of the rotor and blades of a wind turbine [19]

cheap. The efficiency of the solar thermal $\left(1520 \mathrm{~W} / \mathrm{m}^{2}\right)$ based electric power is less than that of photovoltaic $\left(40 \mathrm{~W} / \mathrm{m}^{2}\right)$ based electric power even though the photovoltaic requires less space. This makes it attractive and able to maintain the best output power when land area is limited and the grid is distributed in an array, as shown in Figure $2.6[8]$.

\subsection{Transport Layer Overview}

In the OSI (Open System Interface), the presentation layer converts the application layer file to a configuration that is understandable between the two peers (source and destination). This is because the two peers generally use different types of computer. The transport layer is the heart of the protocol hierarchy and its task is to send and 


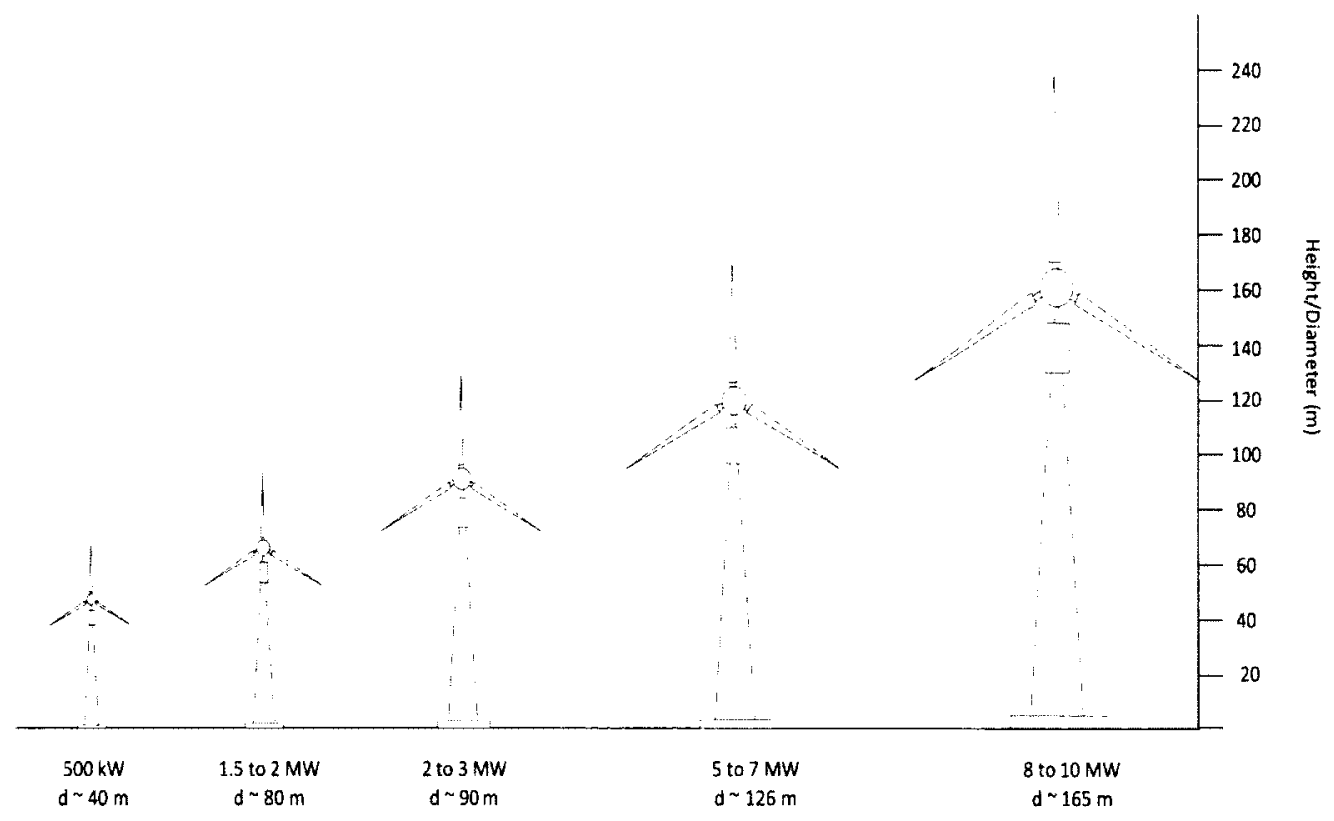

Figure 2.5: Power generated for several sizes of turbines [19]

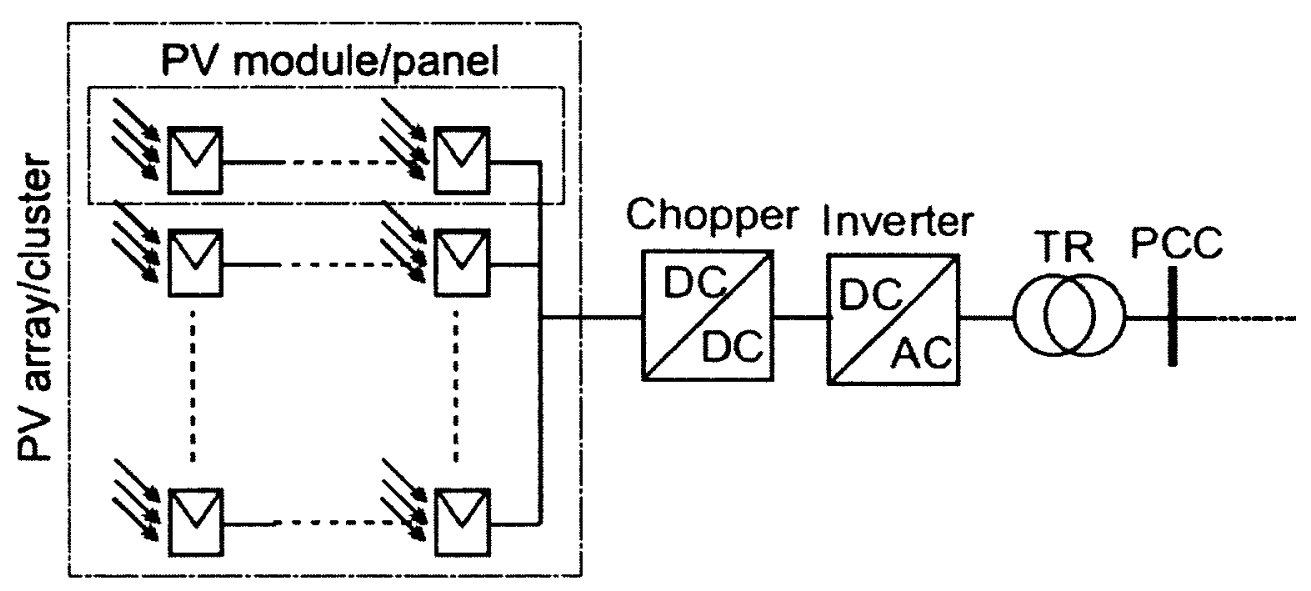

Figure 2.6: Photovoltaic array interface [8] 
control message packets. All transmission protocols, such as TCP, UDP, and SCTP, are controlled and managed in the transport layer [27].

\subsubsection{SCTP Overview}

SCTP is a new and reliable transmission protocol that operates on an unreliable protocol like IP (Internet Protocol). SCTP was introduced and standardized by the Internet Engineering Task Force's (IETF) Signaling Transport (SIGTRAN) working group in RFC 2960 in October 2000. It was then rendered obsolete by RFC 4960 in September $2007[28,29]$. Originally SCTP was created to maintain the signaling of the public switched telephone network (PSTN) when it was connected over an IP network cloud via gateways. This was done so other destinations could be synchronized while never noticing changes due to mixed network clouds. However, SCTP has a superior capability when employed in broader applications. Even though SCTP is similar to TCP in providing a reliable connection-oriented and controlled virtual transmission link, SCTP has more delivery options and alternative features, such as multi-homing and multi-streaming. The association of SCTP to a destination is set up before sending data, and when completed, the destination point terminates the association. The association is set up in a four-way handshake using the cookie mechanism in order to protect the server from any malicious user that may pretend to be as normal user, such as with denial-of-service attacks [9]. As in TCP, the SCTP terminal in the network element, such as the end-user, starts by establishing the SCTP association (SCTP Connection Session in the level of Transport Layer). First the SCTP end-user (i.e. mobile user to be connected to a server) sends an association setup request called the INIT chunk, and the server receives this request but does not assign it or accept it right away. Second, the server replies to this INIT through an acknowledgement called the SCTP INIT-ACK chunk, which includes a cookie that contains all the 
information needed by the sender:

- the details of identification for processing.

- the cookie's life span.

- a signature for security and authentication.

Third, the end-user echoes the cookie back to the server using the SCTP COOKIE ECHO chunk. Finally, the server considers the user information and assigns resources to establish the association. Both client and server now know all the parameters, including the IP addresses in this association, the initial transport sequence number (TSN) for data transfer, the initial tag to be used in the SCTP packet, and the number of outbound or inbound streams each side supports. This is demonstrated in Figure $2.7[9,32]$. It is important to mention that the time delay due to this four-way handshake process is not worse than the three-way handshake of TCP. This is because in the TCP case, when the host receives an association setup request (TCP SYN), the host will immediately allocate resources for the user who sent the request, which is risky in the case of denial-of-service attacks. In these cases the attacker sends the TCP SYN and never replies to the host. This means the allocated resources stay reserved but unutilized $[9,32]$.

\subsection{SCTP vs TCP vs UDP}

As in TCP (RFC793) and UDP (RFC768) [30,31], establishing a connection between a client and a server requires two common things: the IP address of the destination and the port number that determines the application to be used. This same principle is applied in SCTP. The SCTP packet contains both a control part and a data part, but unlike TCP, an SCTP packet can carry more than one message 


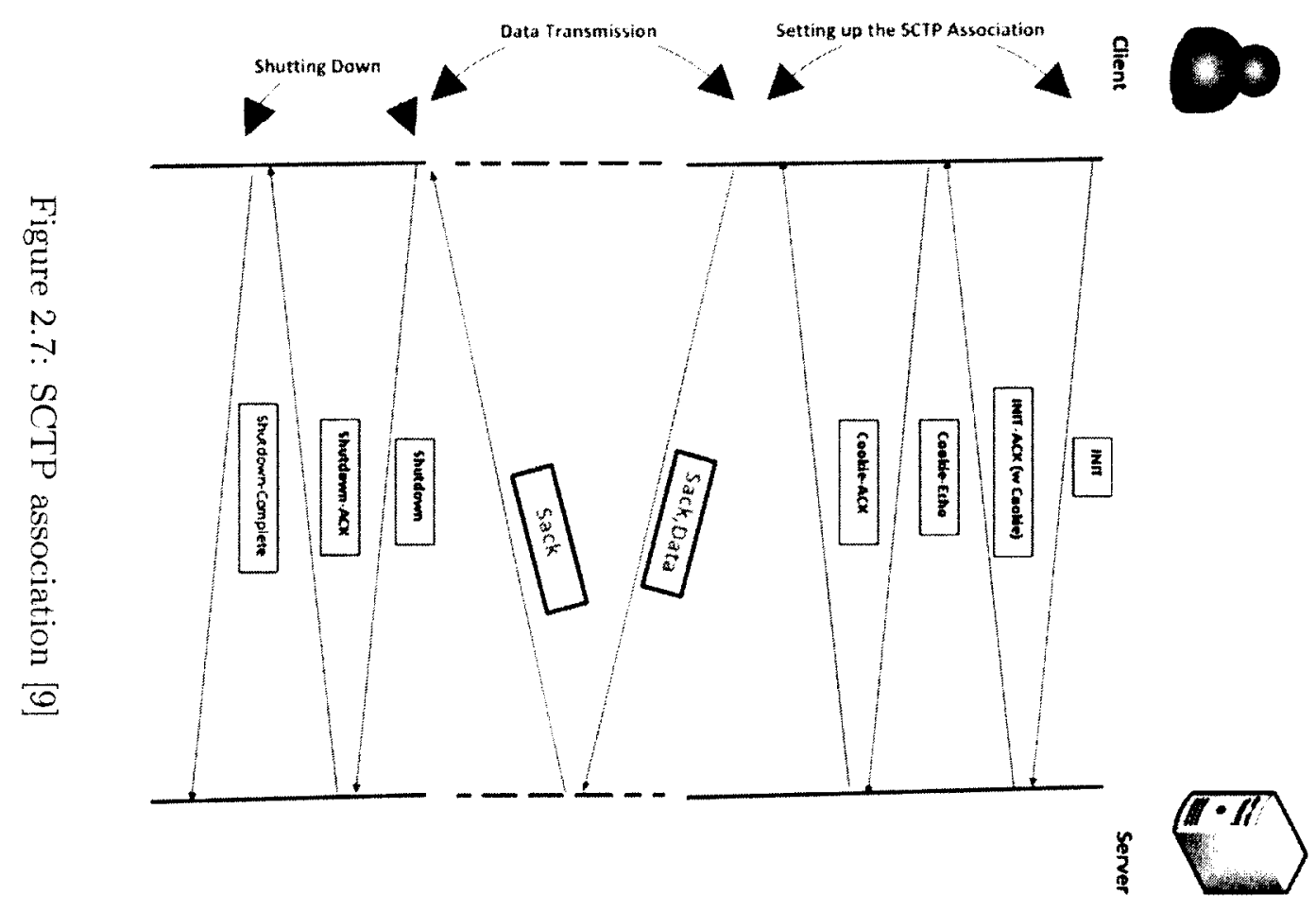


and identifies every message in a data chunk, as demonstrated in Figure 2.8. This means the SCTP host can establish a session with the destination over different IP addresses. That is called multi-homing and is not supported by TCP nor by UDP. This means that if the IP address goes down, a TCP connection is lost and must be re-established. The multi-homing property is very useful in practical field applications like hand-overs. In these cases the SCTP protocol can switch from a primary path to another alternative path when the primary path fails due to any hardware failure.

In addition to the multi-homing property, SCTP also has a multi-streaming feature, allowing the SCTP association to have more than one stream of data chunks transmitting in parallel. For example, transmitting both a video tube and its text content in parallel in a website can be achieved by multi-streaming. This feature confirms the nature of SCTP protocol as a message oriented protocol in which SCTP discovers the maximum transmission unit (MTU) of the path before sending the message. SCTP then makes the required fragmentation on the messages that are larger than the MTU of the path. These fragmented messages are reassembled on the receiver side, which means SCTP association deals with the transmitted data at the message level, not at the byte level as is the case for TCP. In TCP, the association operates and deals with the data as a stream of packets (bytes) to be sent in order one by one. UDP, on the other hand, is a message oriented protocol similar to SCTP but it has no reliability. Table 2.1 demonstrates a good comparison between SCTP, TCP and UDP.

\subsection{Related Work}

SCTP is a new protocol, even for people in the network engineering field. It is expected that SCTP will eventually be the most used protocol due to its capability 
Table 2.1: A comparison of SCTP, TCP and UDP

\begin{tabular}{|c|c|c|c|}
\hline Property & SCTP & TCP & UDP \\
\hline Transmission-orientation & $\begin{array}{l}\text { message } \\
\text { oriented }\end{array}$ & $\begin{array}{l}\text { stream ori- } \\
\text { ented }\end{array}$ & $\begin{array}{l}\text { message } \\
\text { oriented }\end{array}$ \\
\hline Connection-oriented & yes & yes & no \\
\hline Reliability & yes & yes & no \\
\hline $\begin{array}{l}\text { Flow and congestion con- } \\
\text { trol }\end{array}$ & yes & yes & no \\
\hline Unordered data delivery & yes & no & yes \\
\hline Ordered data delivery & yes & yes & no \\
\hline $\begin{array}{l}\text { Multistreaming and Mul- } \\
\text { tihoming }\end{array}$ & yes & no & no \\
\hline Path MTU Discovery & yes & no & no \\
\hline $\begin{array}{l}\text { Protection against SYN } \\
\text { flooding attacks }\end{array}$ & yes & no & no \\
\hline $\begin{array}{l}\text { Dynamic address recon- } \\
\text { figuration }\end{array}$ & yes & no & no \\
\hline $\begin{array}{l}\text { Preservation of message } \\
\text { boundaries }\end{array}$ & yes & no & yes \\
\hline $\begin{array}{l}\text { Application packet data } \\
\text { unit (PDU) fragmenta- } \\
\text { tion }\end{array}$ & yes & yes & no \\
\hline $\begin{array}{l}\text { Application } \quad \text { PDU } \\
\text { bundling }\end{array}$ & yes & yes & no \\
\hline $\begin{array}{l}\text { Allows half-closed con- } \\
\text { nections }\end{array}$ & no & yes & no \\
\hline Reachability check & yes & yes & no \\
\hline
\end{tabular}




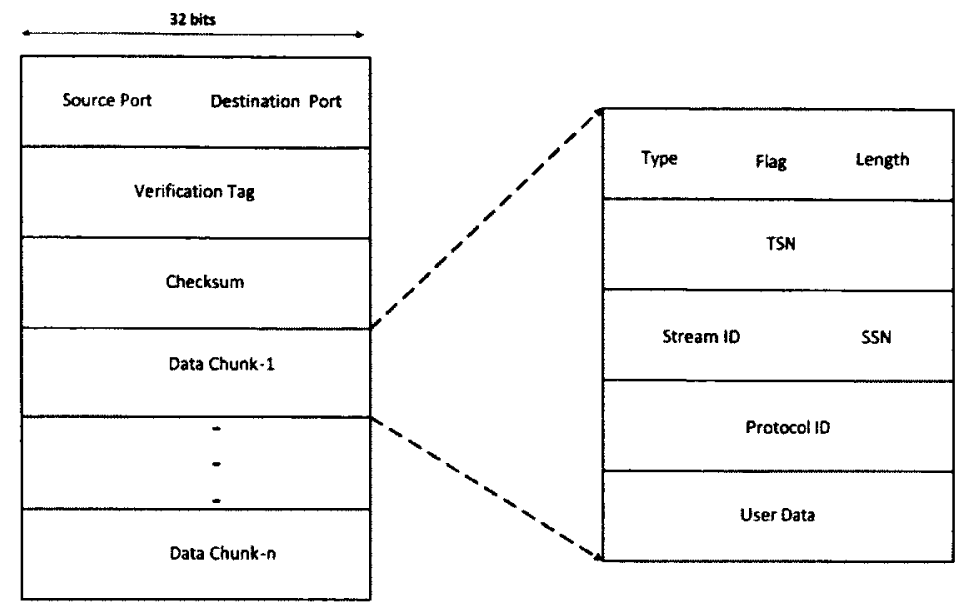

Figure 2.8: SCTP packet format [9]

to do the same job as TCP and more. Because TCP is presently mature and very familiar, some researchers keep employing TCP in order to handle handovers in heterogeneous wide band networks. There is also some other research into using SCTP as an extension to manage the transmission of multimedia (video and audio). The following is a review and summary of some related works that are believed to share many of the same aspects as the proposed system of this work.

\subsubsection{Improving TCP Performance During Intra LTE Han- dover}

In this paper [33] solutions to enhance the quality of FTP traffic during a TCP based handover in the LTE (Long Term Evolution) environment have been introduced and investigated. Since the streaming in the wire-cum-wireless topology is not seamless, it has been shown that using some smart solution techniques can improve the behaviour of the throughput. These proposed solutions include fast path switching. This is where the gateway that connects the wireless cloud with the core network 
(where the servers are available) recognizes a handover command from the sender and makes the switch over to another BS without waiting for the handover confirmation from the receiver.

Another suggested solution is to conduct a handover prediction. The handover start-time can be estimated by using the cross-layer information and checking the bandwidth availability at the destination base station. This estimation is critical because if the prediction is wrong it may cause a disconnect, which goes against the goal of keeping the stream seamless.

The third proposed solution is to use an active queue management approach, which can be achieved by controlling the queue size of the core-network routers where the probabilities for dropping or marking packets is selected. The servers then react with

the marked packets and regulate their sending bit rate. This research is similar to our proposal in that they both use the transport layer to implement an added-value handover process to achieve continuity and high QoS during and after the handover. In contrast, this study only used FTP traffic while our proposed system uses real audio and video traffic in addition to FTP. The other difference is that this proposed system in [33] employs TCP to maintain the handover.

\subsubsection{Analysis of Real-time Multimedia Transmission over PR-SCTP}

Researchers have used a partial reliable SCTP (PR-SCTP) extension to transfer a video stream by analyzing the real-time multimedia transmission over a multi-homing transport protocol [34]. An extension of SCTP protocol called partial reliability extension (PR-SCTP)have been used in two different unsymmetrical paths in order to consider the handover environment and condition, as well as to use different access technology from the source to destination. The multi-homing property of SCTP is 
demonstrated in this work, however the partial reliability extension of SCTP makes it susceptible to packet drop (similar to UDP). Like the LTE models, their proposed topology does not have actual wireless access conditions. Although the link properties were formed in order to behave like wireless access, this research is still similar to our proposal in that they both use video streaming over SCTP. The node structure used in this work uses an infinite buffer size, or in other words, the receiver window is around 100MB. The network congestion is represented by varying the link path loss, which also simulates wireless access without using a true wireless access simulation and true mobile movement. Finally, it appears that this research is again similar to our proposed system in terms of observing SCTP performance when a handover is applied, even though the handover scenario and environment are different.

\subsubsection{SCTP Efficient Flow Control During Handover}

A specific in-progress extension of SCTP called mobile SCTP (mSCTP) is applied in order to support a seamless handover between heterogeneous networks [35]. It has been explained that one of the problem when a multiple network interface node performs a handover of an SCTP connection, the traffic bit rate drops down. This is because of flow control mechanism. To mitigate this problem an efficient flow control mechanism (EFC) over SCTP is applied to minimize the change of traffic rate during handover from the primary path to the alternative path (backup path) and keep the service as seamless as possible during the handover. This can be done by consecrating on three important flow control parameters; Receiver window (rwnd), Congestion

window (cwnd) and Slow-start threshold (ssthresh). The applied approach here is to pre-store the flow control parameters before the handover, to set the flow control parameters during the handover and finally to adjust the flow control parameters after the handover. In other word the idea here is to make the congestion and receiver 


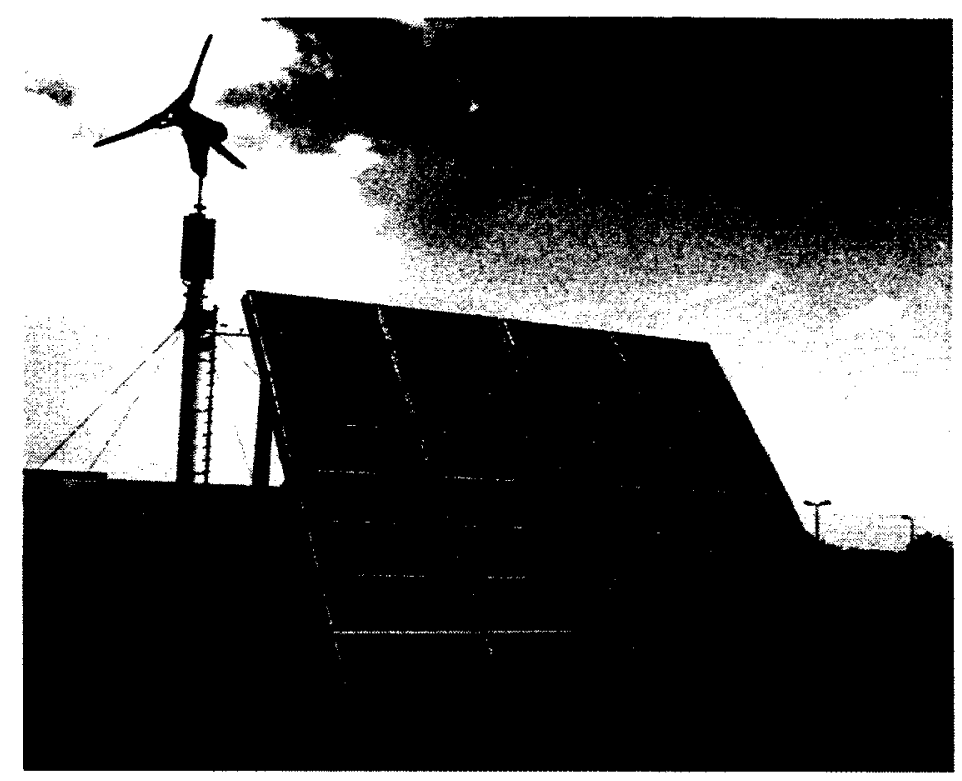

Figure 2.9: Solar and wind combined renewable energy for a cellular network [36]

windows do not start from the initial value which reflects on the bit rate during and after the handover. All these steps are done in order to eliminate the transit-servicecollapse phenomenon. We believe this research is related to ours because it studies the behaviour of the network service when handover using SCTP. However the proposed topology for simulation is full wired and the two nodes (sender and receiver) are directly connected with primary and secondary link even the mobile SCTP is used which may not cover the mobile environment. Also, the traffic here is just FTP stream which is very tolerant to time delay unlike the case of audio and video as it in our work. In addition to that the main goal here is to keep the congestion window as constant as possible during the handover regardless of the delay!. 


\subsection{Summary}

In this chapter, green wireless concepts in telecommunication information technology were introduced. We know that green wireless technology is becoming very urgent in the field today and in the future for both economical and environmental reasons. From an economical point of view, introducing and considering green wireless technology is good motivation for investors (telecom operator or manufacturer) to reduce energy consumption, thereby reducing costs. Moreover, there can be more than one renewable energy source to feed the green cellular base station as shown in figure 2.9. The main environmental goal of green wireless is to reduce $\mathrm{CO}_{2}$ emissions so that the massive tragedy that threatens our planet (i.e. global warming) can be mitigated and controlled. To bring the green wireless initiative into our modern life, the clean and sustainable energy resources mentioned in this chapter have to be used, but some renewable energy resources are more suitable than others. The main factor after availability is the simplicity in using and introducing the renewable energy sources in rural edges of networks, especially for mobile networks. The biggest challenge is to make the end user or the local operator take up the mission of green wireless and contribute in renewable energy adoption. In order to utilize renewable energy resources efficiently, a full reliable SCTP protocol has been intro-

duced. We have explained SCTP's promising features and how SCTP will be a good alternative to TCP or UDP. The two main advantages of SCTP, multi-homing and multi-streaming, have also been explained. In addition, some related researches that employed TCP and SCTP in the handover process have been mentioned. These researches have been criticized to show how it compares to our proposal. In the next chapter we will introduce our green cellular proposal that employs an SCTP-based handover mechanism. 


\section{Chapter 3}

\section{Improved Green Cellular Techniques by Employing SCTP Based Handover Mechanism}

\subsection{Network Topology}

The most typical model for an arbitrary heterogeneous network contains both wired and wireless components and is called the wired-cum-wireless topology. This topology is employed in our simulation to cover the network clouds (e.g. backbone and network access clouds). The main server is located on the backbone, while the mobile user(s) are located on the access side (local small cell access network) as shown in Figure 3.1.

Our solution aims to apply renewable energy gracefully and smoothly. It focuses on the network edges (last mile), not in the network core (backbone). The end customers are typically businesses (not residential) who enjoy services from their service provider and can manage their own property to implement a renewable energy 


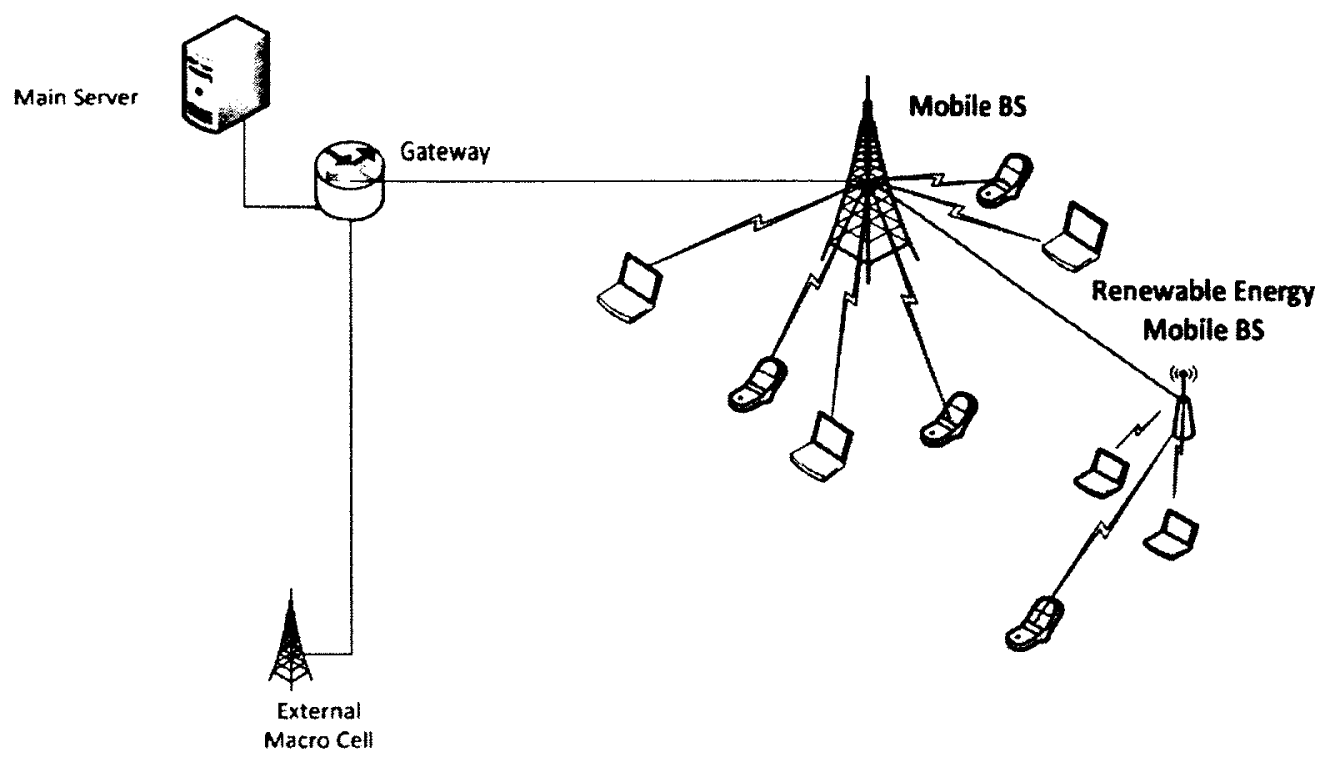

Figure 3.1: Wired-cum-wireless heterogeneous topology

source. In general this customer is connected via DSL (digital subscriber line) or any high broadband connection. We assume that the customer is capable of getting their own renewable energy source constructed. To satisfy this customer's initiative for using this renewable energy property to feed the mobile station with alternative energy, they must be supported regarding the configuration and activation of the alternative base station in all details regarding required IP addresses in the provider network.

\subsubsection{Outdoor Small Cells}

For an outdoor small cell there are three users who are connected to the main access point. This access point is multi-homed to two interfaces. The first interface is a base station (an access point) by itself and is fed by conventional energy. The other interface is the one that is fed by the renewable energy resource as shown in Figure 3.2. In our model we make the user who will be handed over between the bases 


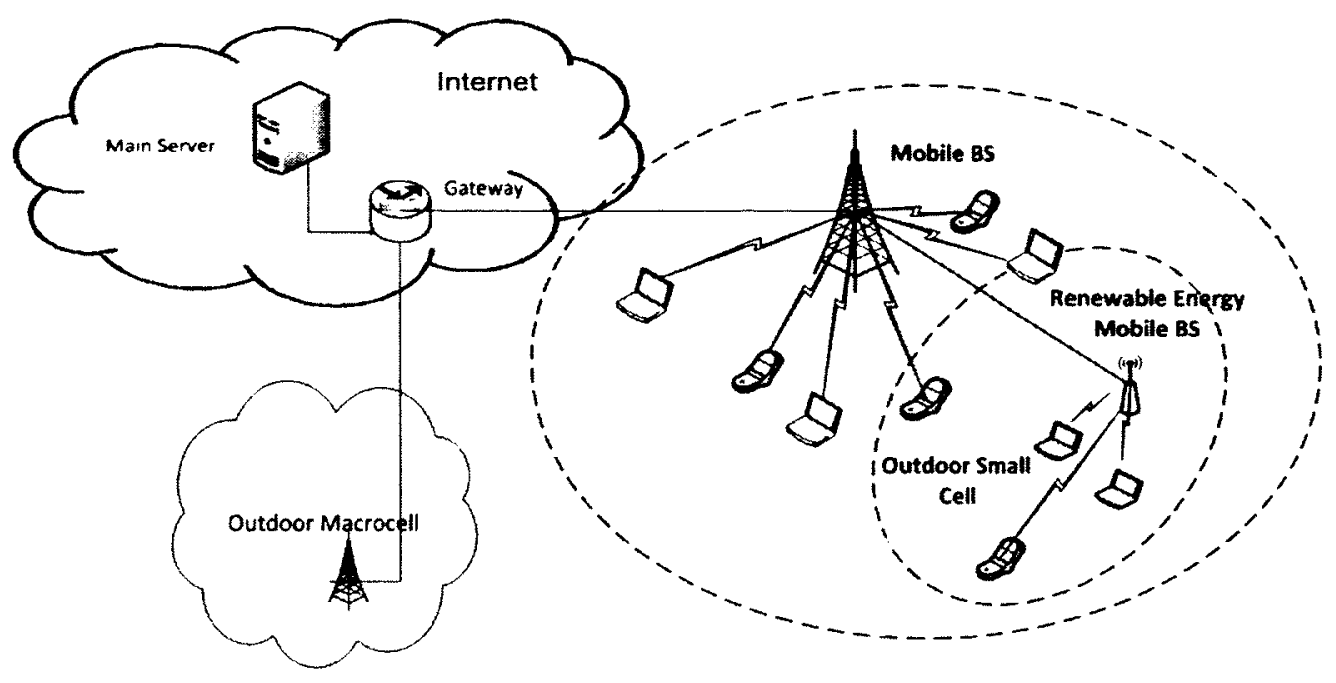

Figure 3.2: Wired-cum-wireless heterogeneous topology clouds

stations move inside the small cell coverage area. The area of the regular macrocell in mobile networks exceeds several square kilometers, and most mobile users in these cells usually move in random directions (called Brownian motion) while they are calling, downloading, or uploading. In the case of picocells or small cell, where the area of the cell is only several square meters, the user movements are approximately straight paths as in Figure 3.3.

In the simulation we assured the user motion is fast, which we set to twenty meters per second. This speed is exaggerated in order to apply tougher conditions on the simulation. This demonstrates the robust behaviour of SCTP and gives the user a chance to move in remarkable positions during the simulation. In addition, the user could be represented by a robot or any fast machine in an indoor area of a factory that needs to be connected to the network for uploading or downloading. 

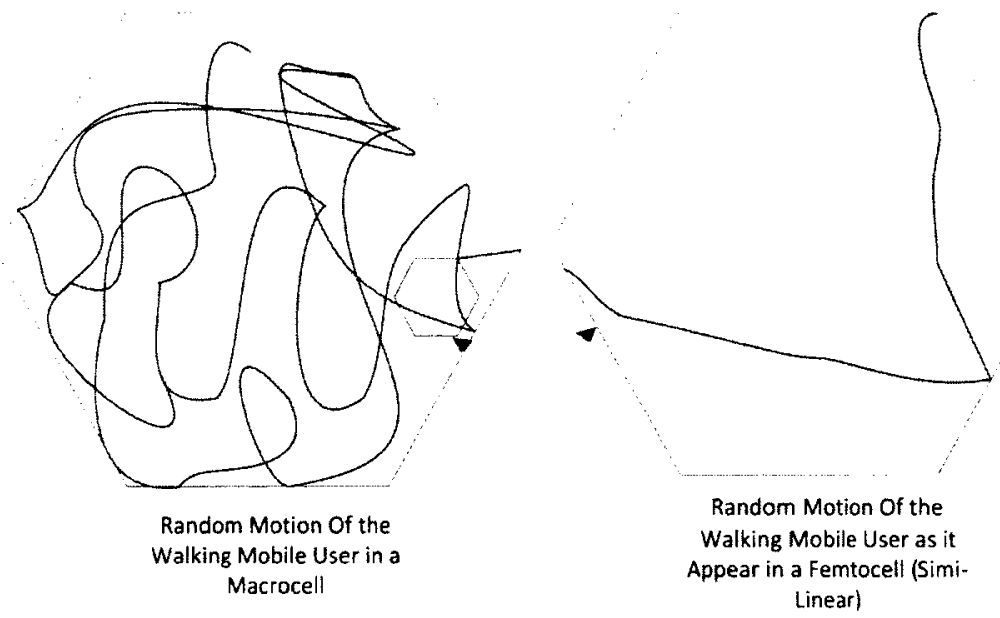

Figure 3.3: Assumption of simi-straight motion with respect to a small cell

\subsection{Heterogeneous Hierarchical Topology Address- ing}

Because the multi-homing principle is applied to the base stations and to the mobile users and because the network topology in this proposal is a mixed network, the simulation requires that the network elements must be addressed hierarchically on the IP addresses. If a uniform addressing is applied, the simulation will not work due to the multi-homing principle. An address error will occur in this case because the classifier in any network element will cause confusion. It will confuse to which direction or ordering it has to sent the message packets or ack packets oppositely. The hierarchic addressing approach uses three digits per node. The first digit indicates the domain, the second digit indicates the cluster in the domain, and the third digit identifies the node itself. For example, the hierarchical address of 1.0 .2 refers to the second node in cluster zero in domain number one. After the nodes have been created and set correctly, any two nodes within the same cluster can be multi-homed to a third node in the same cluster. For example, nodes 0 and 1 can be multi-homed to 
node 2. Those three nodes act together as a single node with two interfaces. In this example, the home node is node 2 and has two interfaces of node 0 and node 1 . The simulation has two domains assigned; one is for the wired side of the topology (two routers) and the other is for the wireless side (the base stations and the mobile users). Hierarchical addresses were assigned to the network elements after dividing them into the two domains (domains 0 and 1 ). Then two clusters were assigned to each domain. In domain 0 a cluster number was assigned for each of the two routers. This means that each cluster in this domain has only one node in it. In domain 1 there were two clusters assigned. In cluster 1 base stations were assigned. In cluster 0 mobile users were assigned. Every mobile user is simulated by three nodes; one home node and two interfaces which means that there are nine nodes in this cluster. In total there are fourteen nodes in the simulated network topology as demonstrated in figure 3.4. It is order to mention that the number of base station is two in the topology, there are the conventional energy fed base station (hierarchical address of 1.1.1) and the renewable energy fed base station (hierarchical address of 1.1.0). The multi-homed mobile base station (hierarchical address of 1.0.0) does not play as an access base station. This node is just introduced for simulation purposes where we need three nodes to make the multi-homing connection but this node does not play any mobile access role $[37,38,54]$

\subsection{Simulation Environment and LTE Models}

The long term evolution (LTE) properties are modeled and patched/injected to the links in the simulated network topology. Performance of the transmission protocol (TCP or SCTP) over LTE is affected mainly by the links of the wired network [55]. The LTE models are patched to the wired side of the network since the wireless side is merely an access. The handover effect occurs with respect to the wired side where 


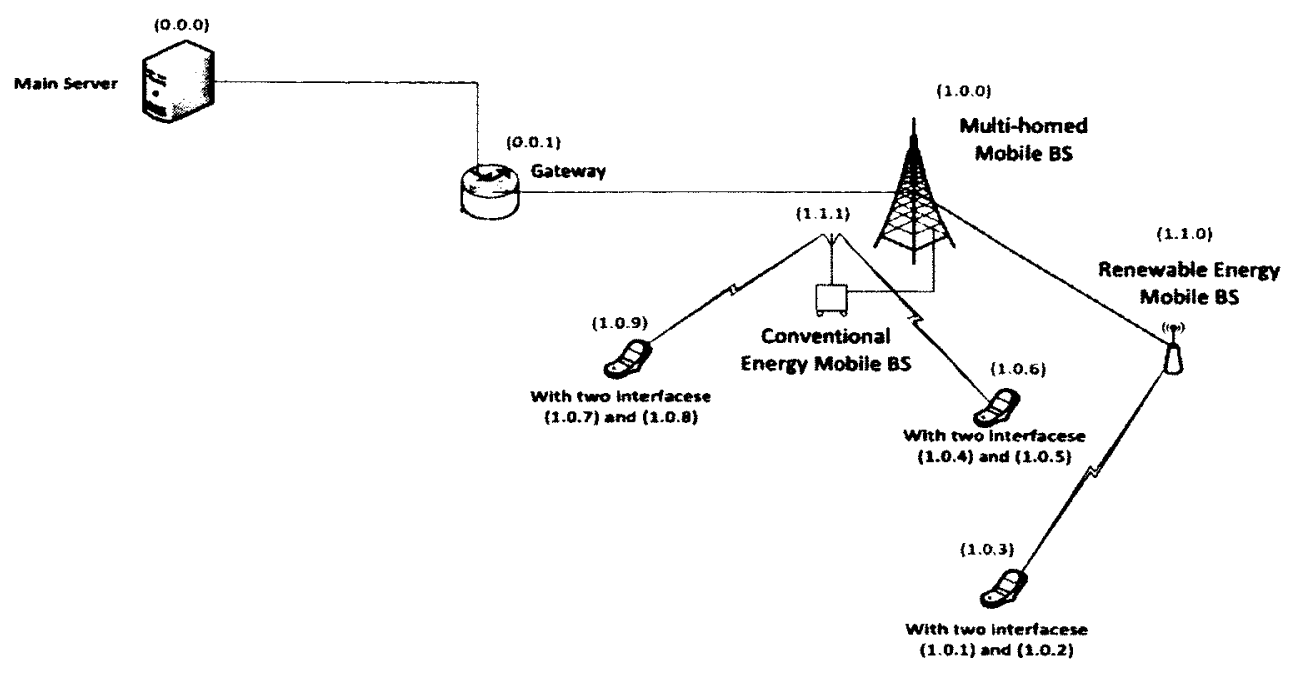

Figure 3.4: Hierarchical addresses of the network elements

the traffic is classified due to the QoS priority.

The coverage area of the renewable energy base station is expected to be less than that of the conventional energy base station. This is maintained by reducing the transmission power of the renewable energy base station. Also, this renewable energy base station is subjected to fluctuations and is considered unstable. In our simulation we assume that the renewable energy base station is in an up threshold in the beginning of the session, then at random it drops under the threshold. This means that the user will be handed over to the conventional energy base station and the power of the renewable energy base station can exceed the threshold randomly to be handed back to the renewable energy base station. The handover scenario here is the availability of the alternative energy power source. If this energy source attenuates or decays at any time, then the decision is to hand off the user to the conventional energy base station as shown in figure 3.5 . The simulation time is 20 seconds. 


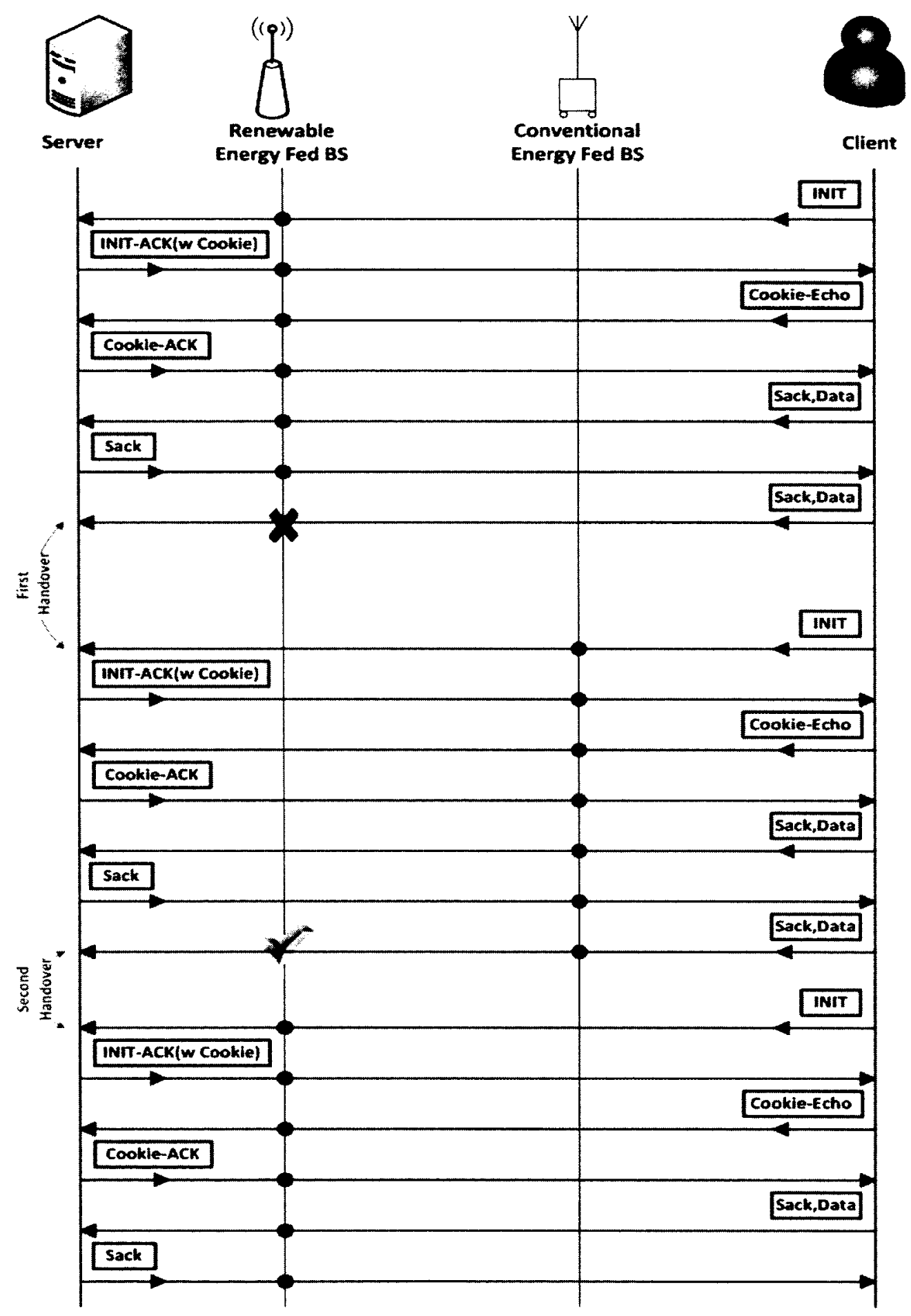

Figure 3.5: The handover procedure 


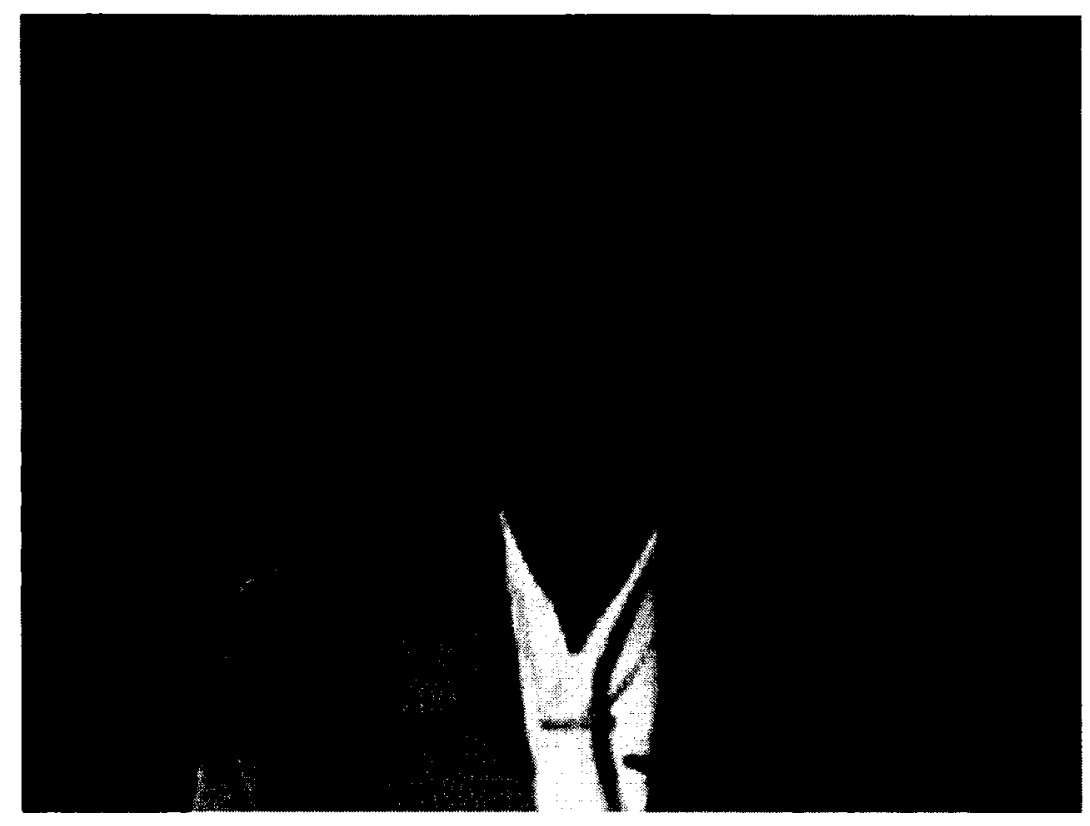

Figure 3.6: YUV video sequence-352×288 Resolution

\subsection{TCP and SCTP Agents and the Hierarchical Addressing}

The TCP agents are attached to the user endpoint with the hierarchical address of 1.0.3, and the network server with the address 0.0.0. The connection between the TCP source and sink is at the transport layer level regardless of the MAC layer or physical layer type. At the handover time the user is handed off from the renewable energy base station to the conventional energy base station. For the second handover time the user is handed off back from then conventional energy base station to the renewable energy base station. This approach is applied also for the case of SCTP. In this case one SCTP agent is attached to the source node and another SCTP agent is attached to the destination node, effectively connecting the two SCTP agents. 


\subsubsection{Handover Scenario and Traffic}

The simulation progress and important events are now described. At the beginning of the simulation, the transmitting power of the renewable energy base station is set to be $40 \%$ with respect to the default power of the conventional energy base station. The traffic starts at time $t=3$ seconds, and at a random time $t=5.02$ seconds the power of the renewable energy drops so that the first handover occurs. At this instant, the user is handed off to the conventional energy base station. At time $t=8.95$ seconds, the power of the renewable energy base station comes again to $20 \%$ of the default value so that the second handover occurs at this time while the mobile user is moving. To reflect the fluctuating variance of the renewable energy base station, at time $t=$ 9.7 seconds the renewable energy power is set to be $40 \%$ in of the default value, then by time $t=16.5$ seconds it is adjusted to be $60 \%$ of the default value. It is order to say that the first handover instant has been chosen due to the random drop of the renewable energy source that feeds the renewable energy base station and the second hand over instant has been chosen due to the random rise of the renewable energy source that feeds the renewable energy base station. So that, the handover instants have been selected in random. In our case, where we want to utilize the renewable energy resource as much as possible, the handover decision is made only when the renewable energy fed base station becomes out of energy (i.e. down case), so the prediction of handover time (as in [39]) is not applied here.

The applied traffics here consist of three types:

- FTP (File transfer protocol) is a traffic stream which may, for example, be a file downloading from or uploading to the internet by the user. In this type of traffic the traffic delay is insignificant because it does not affect the user's convenience compared to audio or video traffic.

- Real audio traffic with a constant bit rate. This audio is adjusted for peer-to- 
peer conservation. so this traffic is very sensitive to the delay that may occur in the network.

- Real video traffic stream that simulated a live video transmission. In this case a 10 second length video stream is transmitted to simulate a live video watching over the network. This file was in a YUV format at its origin is from a video trace library on the web that contains such standard video sequences that researchers use to test protocol capabilities in carrying video sequences [42]. This video file(Akiyo) shows a TV news anchor while she is speaking and the video contains 300 frames as shown in figure 3.6. We converted this video format from yuv to MP4 format to make it suitable to be sent via simulated network. This required patching to our default simulation tool to make the video able to be carried via TCP and SCTP $[43,44]$.

In such wire-cum-wireless environment like our topology, the comparison between SCTP and TCP to carry the three types of traffic (FTP, Audio and Video) will be conducted for specific criteria as will shown in chapter four. We choose to make the comparison between SCTP and TCP not between SCTP and UDP even the UDP is usually used to transport multimedia traffic. This is because the UDP has no congestion control feature so it will work without any care of the network condition which will consume the network limited bandwidth. Therefore TCP used to be considered in the wireless network $[40,41]$. In addition to that the natural of wireless access network as ours, required some degree of security to distinguish the malicious user who may access the network offside. SCTP have been used for maintaining the handover, but the purposes and the conditions is quite different. Unlike the other previous works, in this work the environment is true wireless and the users (especially the user to be handed over between the base station) are moving. Also the hand over purposes and scenario is employed to save the energy and use the renewable energy as much as 
possible because we consider a fluctuating renewable energy resource. Therefore, the handover is subjected to occur many times in a short period. In addition to that, the other new thing is that the Long term evolution (LTE) environment is considered in this work simulation.

\subsection{Summary}

In this Chapter we have introduced and explain our proposed solution called $\mathrm{Im}$ proved Green Cellular Technique by Employing SCTP Based Handover Mechanism. The network topology to be used is demonstrated and verified. Also, the nature of mixed networks that contain wired and wireless components and the challenge of configuring the addresses hierarchically instead of using a flat addressing mode have been explained [45]. The proposed solution considers the natural condition and the user movement taking the LTE environment in account. Tougher conditions were added to the network to highlight the advantages of SCTP, such as multi-homing. A model for the renewable energy access point was introduced, along with how it changes to analogize the renewable energy source practically. Also the user movements inside the small cell and the time instants of handovers due to the drop in renewable energy feeding the access point have been configured. Several traffic streams have been chosen to be used in the simulation. It is only a matter of configure the simulation tool to deal with such traffic streams, especially the video traffic. It is also necessary to reformat the video to make it possible to be attached to the SCTP or TCP agents. In this next chapter the simulation tool and results will be discussed in detail. 


\section{Chapter 4}

\section{Simulation Results and Discussions}

\subsection{Network Simulation Tools}

In engineering, simulation processes apply representative, time-variable, physical conditions to a target system. Networking is a good example of an engineering system where the events and variables (e.g. arriving packets or error occurrence) vary with time in probabilistic quantities. No closed deterministic mathematical model can describe or represent those quantities, so researchers use simulation to mimic the processes that occur within that system. In nature the events can be continuous or discrete. Discrete events (our case) might be computer network packets transmitting from the source, arriving at the destination, or queuing in a buffer.

All the network elements in any computerized distribution system are discretetime machine. It is very similar in concept and analogy when solving the differential equations in a closed form (for all time values). It is challenging, but one can surpass this difficulty by applying a numerical methods, or employing mathematical identities that give the solution of the differential equation at discrete time intervals (for example every $1 \mathrm{~ms}$ or $1 \mu \mathrm{s}$ ). The same concept is applied in the discrete simulation tools. 
There are several discrete event-based simulator tools that can be used for network simulation [46]. There are some practical commonly used simulation tools such as OPNET, Network Simulator 2 (ns-2), OMNet++, QualNET, SSFNet and J-Sim.

Usually when choosing a simulation tool or package, researcher must take into consideration the properties and features for the selected tool to go with. The properties to examine depend on the network environment (topology and protocol) and simulation objectives that the researcher wants to attain (e.g. scaling network delay or observing quality of service). The simulation tool characteristics must intersect with what the researcher is interested in. To specify and choose the most suitable simulation tools for our needs, the following parameters and features were taken into account [47]:

- The availability and accessibility of the simulation tools software is very important. This includes how easy it is to attain the software and whether it is open source (free licence) or commercial.

- The environment/the operation system that the simulation software is hosted in has to be considered. The software must be compatible with the operation system such as Windows, Mac OS x or Linux (Linux Kernel).

- The supported protocols related to such network layer to be tested have to be included in simulation tool software package. For example, in our case we have to use such software that support the TCP and SCTP protocols. This takes in consideration the flexibility of the modules and how rich the protocols libraries are.

- The flexibility of the simulation tool and its ease of use have to be in mind. This deals with the user interface of the simulation tool either it has GUI (graphic 
user interface) property or it has a simple script editor (command user interface) to interface with $[49,50]$.

\subsubsection{Simulation Tools Survey and Comparison}

The following presents a brief summary about the most common simulation tools that researchers and students normally use in network simulation and how the most suitable one was selected for our intended work [51].

Table 4.1: Key Differences between Simulation Tools

\begin{tabular}{|l||l|l|l|l|l|l|}
\hline \multicolumn{7}{|c|}{ Network Simulators Comparison } \\
\hline $\begin{array}{l}\text { Programming } \\
\text { Environment }\end{array}$ & C++/OTCI & C++ & Java/Jacl & C++/NED & C-Based & C++/C \\
\hline $\begin{array}{l}\text { Graphical In- } \\
\text { terface(GUI) }\end{array}$ & No & Yes & Yes & Yes & Yes & Yes \\
\hline $\begin{array}{l}\text { License } \\
\text { Needed }\end{array}$ & No & No & No & No & Yes & Yes \\
\hline $\begin{array}{l}\text { Support Mo- } \\
\text { bility }\end{array}$ & Yes & Yes & Yes & Yes & Yes & Yes \\
\hline $\begin{array}{l}\text { Support } \\
\text { TCP/UDP }\end{array}$ & Yes & Yes & No & Yes & Yes & Yes \\
\hline Support SCTP & Yes & No & No & Yes (Basic) & Yes & No \\
\hline $\begin{array}{l}\text { Documentation } \\
\text { and Support }\end{array}$ & Excellent & Poor & Poor & good & good & excellent \\
\hline
\end{tabular}

As shown in table 4.1 the network simulator (NS-2) provides and satisfies the required properties which help to conduct the simulation of our solution (Improved Green Cellular Technique by Employing SCTTP Based Handover Mechanism). In the next subsection, the ns- 2 construction will be introduced and work theory will be 
discussed in detail.

\subsubsection{NS-2 Simulation Tool}

Network simulator (NS-2) was developed at the University of California-Berkeley. Ns-2 is considered to be the standard network simulation tool. More than $50 \%$ of IEEE papers cite ns-2 as simulator in the field of modelling behaviour of network nodes or links [52]. Ns-2 is implemented based on $\mathrm{C}++$ and OTcl (Object-Oriented Tool Command Language). The $\mathrm{C}++$ part (core part) is an object oriented simulator, and the OTcl part (front part) possesses the interpreter that executes the command scripts. As an example, the network nodes and links construction (i.e. Network Topology) in addition to the protocol agents (e.g. SCTP or TCP) are established and assigned by the OTcl scripts [53] while the events (e.g. sending, receiving, dropping, etc.) on this topology are processed by $\mathrm{C}++$.

The advantage of using these two programming languages is that each language has a property that the other lacks. $\mathrm{C}++$ is very strong in performance, but it is very difficult to modify it gracefully, which is required in protocol designing or testing because there is no easy visual interface. On the other hand, OTcl has a simple user interface that helps the user to modify or redesign the network components and agents.

To take advantage of each language's strength, network components and protocols are described and complied by $\mathrm{C}++$, and due to the strength of $\mathrm{C}++$, the processing time is optimized. OTcl, on the other hand, describes network topology and applications (e.g. traffic agents), which means changing or modifying network conditions is made easy. When the simulation is finished, the output results can be provided either as text-based or animation-based information. To visualize the animated results, the NAM (Network AniMator) tool can be used, as demonstrated in Figure 4.1.

In our proposal we used ns-2.33 (NS-2 version 33) which was released in March 

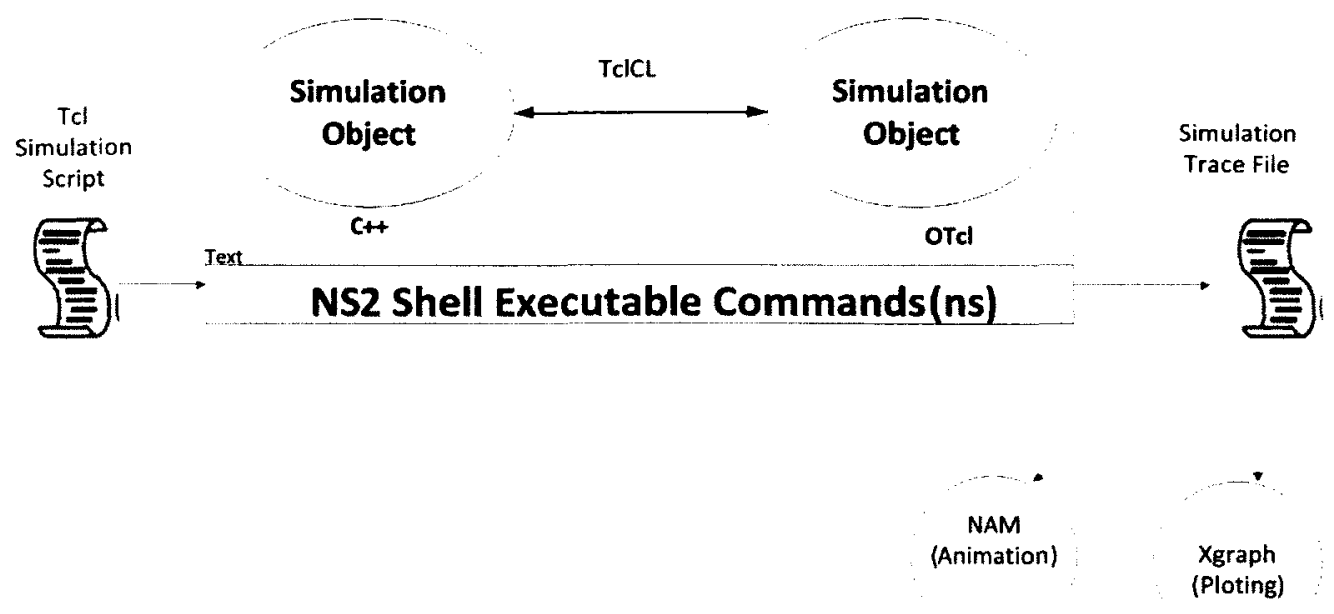

Figure 4.1: Basic architecture of ns-2 [53]

$2008[37,54]$ and we ran it on Ubuntu Linux distribution environment version 9.10. We mean to use ns-2.33, and not to use ns-2.34 because the LTE model (for Ns-2) is compatible only for ns-2.33 [55]. We got the LTE-model from [55] and we patched it to ns-2.33. After that, we did all the required compilation and reconfiguration such as re-installation and re-make as follows:

cd /home/abdullah/ns-allinone-2.33/tk8.4.18

patch - po $<$ tk-8.4-lastevent.patch

cd ... /

./install

cd ns-2.33/

svn checkout http://1te-model.googlecode.com/svn/trunk/

mkdir project \% to make a new directory

cd lte-model-read-only/

sh checkin

cd .../ 
\%After that the pathes in MAKE file must be changed to be consistant

$\%$ with the user machine, so the Makefile must be re-edit

$\%$ all /root/ns-allinone-2.33 must be changes to your local path

$\%$ for examle mine /home/abdullah/ns-allinone-2.33/ in the Makefile

$\%(8$ times edit)

$\%$ last step

Make

To simulate the renewable energy variation, we have made the required modification for the file wirless-phy.cc to make the transmission power of the renewable energy base station can be controlled from the TCL script with time to reflect the power fluctuating. Finally to simulate the video stream which is not available in the ns-2 typical package, we have used the evalvid package [56] which is a framework designed to evaluate the video transmission. To make evalvid work with ns-2 we use the modified package in [57]. This package is implemented to be used for MPEG video transmission over UDP then the received frames can be reconstructed again and compared with the original video. This extension of evalvid in [57] has a modified source code for UDP (udp.cc) that take the reconstruction in consideration and recognize the frame types and position, however the evalvid tool can be employed also for TCP and SCTP in the transport level because the evalvid tool can extract the video frames and put them in a trace file then these frames can be sent over TCP ar SCTP agent but the received frames can not be reconstructed to assembly the video because some modifications must be done to the source code of tcp.cc and sctp.cc in the ns-2 package which is out of our research purposes and considered to be in the future works.

In order to send the video through our simulated ns-2 network, we have to convert 
yuv to. $\mathrm{m} 4 \mathrm{v}$ using ffmpeg (compressed video with 30 frames per second):

ffmpeg -s cif $-\mathrm{r} 30$-b 64000 -bt 3200 -g 30 -i akiyo_cif.yuv -vcodec mpeg4 a02.m4v

Then we have to add a hint track the video track and converting it to .mp4 :

MP4Box -hint -mtu 1024 -fps 30 -add a01.m4v a02.mp4

At the end we use the produced compressed file to extract the sender trace file (which is equivalent to MP4). Then we have to "hint" the video track by converting it to "M4V". This hinted M4V file is used to extract the sender trace file (ST_a01) that contains frames ID size and number of packet per frame. This sender trace file is the file to be used with ns-2 and TCP and SCTP agents can deal with it (i.e. can be transmitted over TCP and SCTP in ns-2):

mp4trace -f -s 192.168.0.2 12346 a01.mp4 > st_a01

Ns-2 supports transport layer protocols by default. This includes signalling, transport and control protocols such as TCP, UDP and SCTP. For the first and second protocols the source node is attached to the protocol agent and the destination node is attached with the protocol sink. Both agent and sink are objects and has their default parameters in the TCL library. The output of the simulation comes in a tr extended (troff document) file which is generated by ns-2 to demonstrate time events during the simulation. For the wired part of the network the output trace file of TCP protocol comes and organised as shown in table 4.2. For the wireless part, the formate is a bit complicated and contains 21 columns instead of 12 as in the case of wired part as shown in table 4.3. For the SCTP protocol, the output trace file is as presented in table 4.4 and table 4.5 for the wired and wireless part respectfully. As shown in table 4.4 the SCTP cares about the stream ID due to the multi streaming capability 
Table 4.2: Trace File Format for TCP

Column no. and Details

\begin{tabular}{|l|l|l|l|l|l|l|l|l|l|l|l|}
\hline 1 & 2 & 3 & 4 & 5 & 6 & 7 & 8 & 9 & 10 & 11 & 12 \\
\hline Event & Time & $\begin{array}{l}\text { From } \\
\text { Node }\end{array}$ & $\begin{array}{l}\text { To } \\
\text { Node }\end{array}$ & $\begin{array}{l}\text { Pkt } \\
\text { Type }\end{array}$ & $\begin{array}{l}\text { Pkt } \\
\text { Size }\end{array}$ & Flags & $\begin{array}{l}\text { Flow } \\
\text { ID }\end{array}$ & $\begin{array}{l}\text { Src. } \\
\text { Add. }\end{array}$ & $\begin{array}{l}\text { Dst. } \\
\text { Add. }\end{array}$ & TSN & $\begin{array}{l}\text { Pkt } \\
\text { ID }\end{array}$ \\
\hline
\end{tabular}

Table 4.3: Trace File Format of TCP for the Wireless Part

\begin{tabular}{|l|l|l|l|l|l|l|l|l|l|l|l|l|}
\hline \multicolumn{10}{|c|}{ Column no. and Detail } \\
\hline \hline 1 & 2 & 3 & 4 & 5 & 6 & 7 & 8 & - & 18 & 19 & 20 & 21 \\
\hline Even & Time & $\begin{array}{l}\text { From } \\
\text { Node }\end{array}$ & $\begin{array}{l}\text { MAC } \\
\text { Action }\end{array}$ & Flag & $\begin{array}{l}\text { Pkt } \\
\text { ID }\end{array}$ & $\begin{array}{l}\text { Pkt } \\
\text { Type }\end{array}$ & $\begin{array}{c}\text { Pkt } \\
\text { Size }\end{array}$ & - & TSN- & - & - & - \\
\hline
\end{tabular}

of SCTP. We have run the simulation several time (more than 20 times) for each case (each traffic type ) to make it match reality as possible. We have found the simulation result and graph envelop is similar and no much difference in result such as delay or throughput (in the order of $0.1 \mathrm{~ms}$ in instantaneous delay and in variance of 3 to 5 Kbps in the instantaneous throughput for audio and in the level of .1 Mbps for FTP and video traffic). This is make since when looking to ns2 as a discrete event and there are a lot of events (the number of packets per second). In additional to that the simulation step is less than 0.001 second which makes the stochastic model behaves in precision and get enough chance to recover any accumulating quantity [58]. We have employed the AWK $[59,60]$ software to catch and filtrate the results out of the output trace file (.tr file). For plotting, we have used GNUPLOT [61]. 
Table 4.4: Trace File Format for SCTP

Fields no. and description

\begin{tabular}{|l|l|l|l|l|l|l|l|l|l|l|l|l|l|l|}
\hline 1 & 2 & 3 & 4 & 5 & 6 & 7 & 8 & 9 & 10 & 11 & 12 & 13 & 14 & 15 \\
\hline Event & Time & $\begin{array}{l}\text { From } \\
\text { Node }\end{array}$ & $\begin{array}{l}\text { To } \\
\text { Node }\end{array}$ & $\begin{array}{l}\text { Pkt } \\
\text { Type }\end{array}$ & $\begin{array}{l}\text { Pkt } \\
\text { Size }\end{array}$ & Flags & $\begin{array}{l}\text { Flow } \\
\text { ID }\end{array}$ & $\begin{array}{l}\text { Src. } \\
\text { Add. }\end{array}$ & $\begin{array}{l}\text { Dst. } \\
\text { Add. }\end{array}$ & N/A & TSN & $\begin{array}{l}\text { Pkt } \\
\text { ID }\end{array}$ & $\begin{array}{l}\text { Strm } \\
\text { ID }\end{array}$ & SSN \\
\hline
\end{tabular}

Table 4.5: Trace File Format of SCTP for the Wireless Part

\begin{tabular}{|c|c|c|c|c|c|c|c|c|c|c|c|c|}
\hline \multicolumn{13}{|c|}{ Column no. and Detail } \\
\hline 1 & 2 & 3 & 4 & 5 & 6 & 7 & 8 & - & 19 & 20 & 21 & 22 \\
\hline Even & Time & $\begin{array}{l}\text { From } \\
\text { Noded }\end{array}$ & $\begin{array}{l}\text { MAC } \\
\text { Action }\end{array}$ & Flag & Pkt & Pkt & $\begin{array}{l}\text { Pkt } \\
\text { i }\end{array}$ & - & Data & TSN- & - & - \\
\hline
\end{tabular}

\subsection{Results of TCP and SCTP based Handovers}

\subsubsection{FTP Traffic}

After running the simulation for the FTP traffic for two handover times between the renewable energy base station and the conventional energy base station, the output result is displayed in Figure 4.2. As shown in Figure 4.2 the first handover instant (at the time of 5.02 seconds) the stream of TCP stopped and it took around one second to continue after TCP managed the handover to the conventional energy access point. At the second handover instant (at the time of 8.9 seconds) the handover took a little bit lower time to manage the handover back to the renewable energy access point. For SCTP stream, the superiority of the SCTP performance can be shown in term of good behaviour to the handover (very low delay during the handover and more TSN). It can be shown that some of the packets after the first handover have been lost and when the sender did not receive any ack, it sent them again and that explain 
the disturbance of the TSN graph of the SCTP. At the second handover instant, SCTP stream seams very smooth and we believe the reason is that the user is closer to the tower and the handover is from the conventional energy access point to the renewable energy access point so both of them are on in this handover instant. The instantaneous throughput is evaluated from the output trace file and figured out in Figure 4.3 .

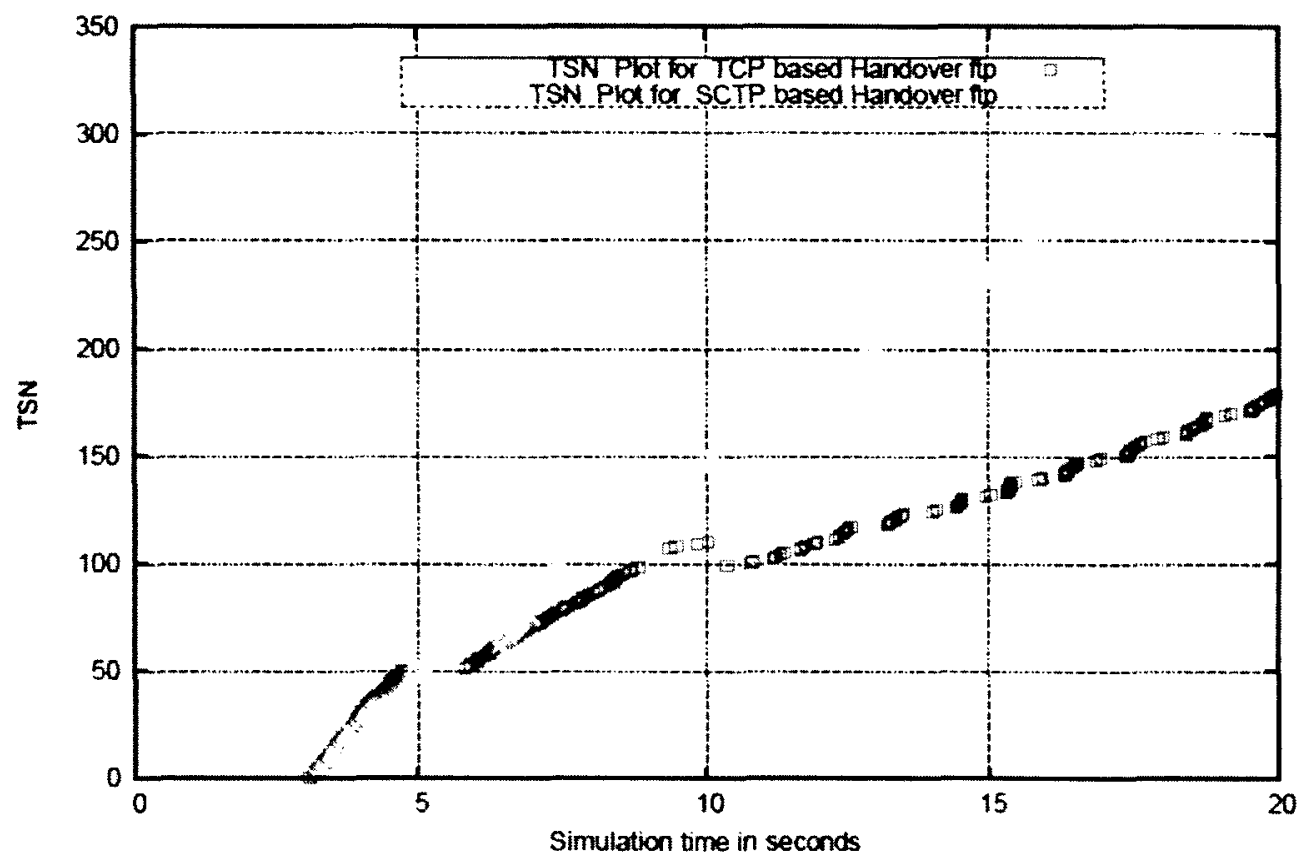

Figure 4.2: SCTP vs. TCP TSN behaviour for FTP traffic

As Shown in this figure, the SCTP throughput increases in a logarithmic scale smoothly and did not like be affected by the first handover expect some little disturbance until it saturated around $1.9 \mathrm{Mbps}$. The TCP throughput in this figure got a disconnection after the first handover, then it increased Significantly until the second handover (from the conventional energy access point to the renewable energy access 
point). It then decreased and saturated around one Mbps. This decreasing in the TCP throughput consist with the relative less energy of the renewable energy access point. Finally, the instantaneous delay is evaluated from the trace file is presented in Figure 4.4. From this figure, it is clear that the instantaneous delay behaviour of SCTP is around 0.25 seconds on average, although it reached around 0.8 seconds at the first handover. The TCP instantaneous delay reaches more than one second at the first handover, but after that it is less than 0.2 seconds in most of the packets but it is more than one second in some few packets. However there is a trade off between the instantaneous delay and the number of packets that have been sent, which clarifies that the SCTP delay is due to the higher number of packets that have been sent as compared to TCP.

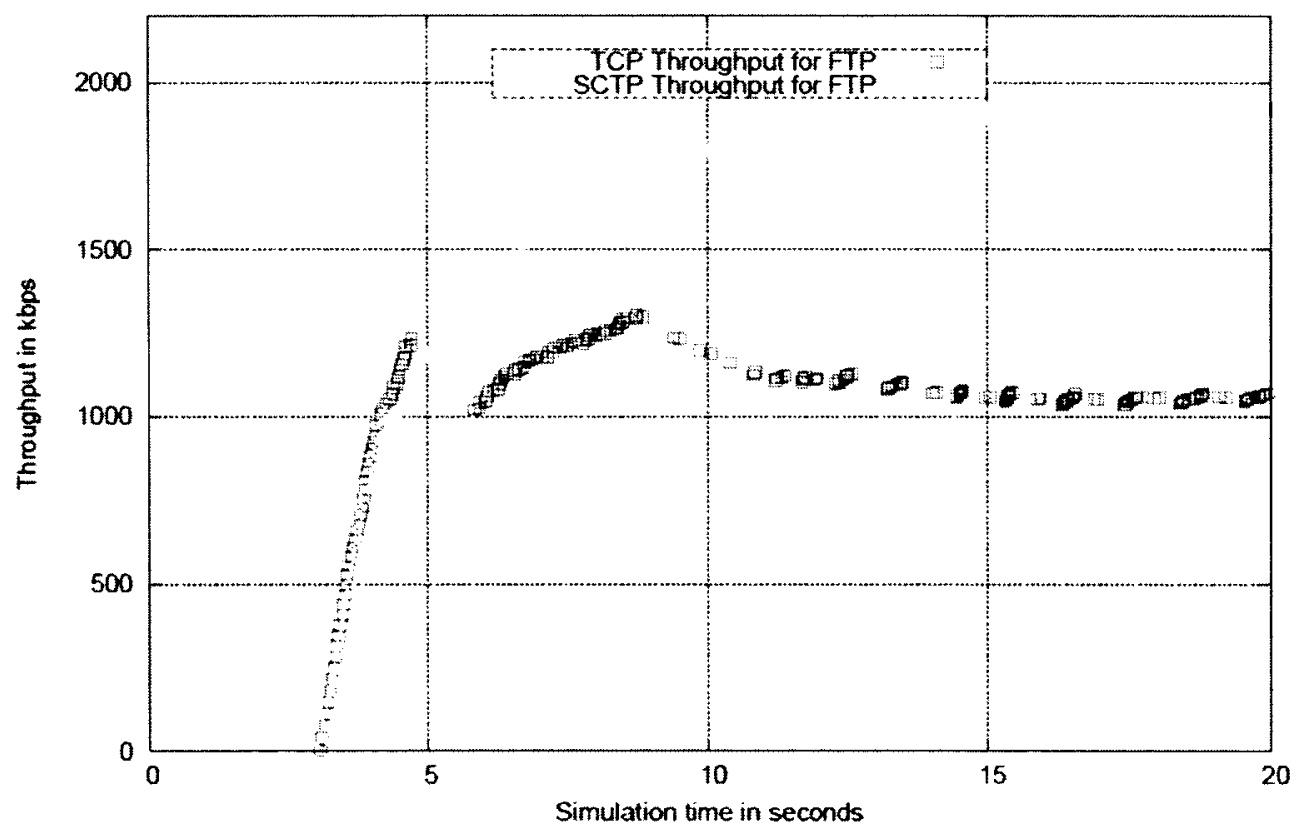

Figure 4.3: SCTP vs. TCP instantaneous throughput behaviour for FTP traffic 


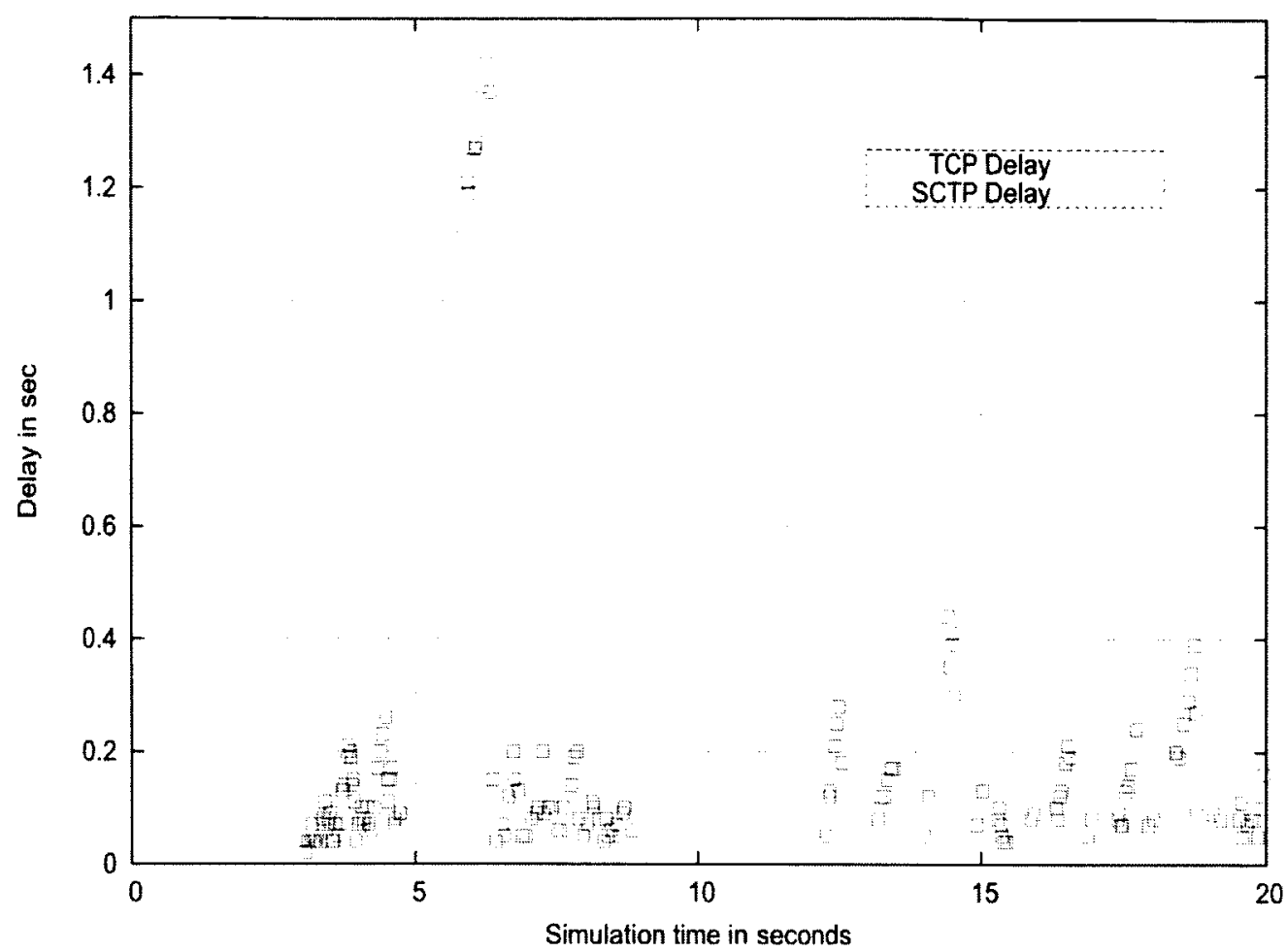

Figure 4.4: SCTP vs. TCP delay behaviour for FTP traffic

\subsubsection{Audio Traffic}

A simulation for audio traffic was also run. Again, there were two handovers between the renewable and conventional energy base stations. The TSN output results are presented in Figure 4.5. In this case, the audio traffic is assigned to be peer to peer conversation. The LTE class in this case is assigned to be class 0 which means the priority is for the audio traffic. As demonstrated in Figure 4.5 the TSN plot for the TCP reached to just $16 \%$ out of the TSN plot of SCTP approximately. This shows the capability of SCTP to carry the audio traffic even in the existence of other traffic that comes of other two mobile users in the network as shown in the topology in chapter 3 . The instantaneous throughput is evaluated from the output trace file and 
presented in Figure 4.6. As in this figure, the throughput of SCTP reached about 85 Kbps while it is around $25 \mathrm{kbps}$ in the case of TCP. Finally the instantaneous delay have been evaluated from the trace file as presented in Figure 4.7. As in this figure, the instantaneous delay in the case of SCTP is 0.3 seconds maximum while it is a little bit better behaviour in the case of TCP but at the expense of number of packets that have been sent. In other word a smaller number of audio sessions can be served in the case of TCP as compared to SCTP. The reason of the delay difference being not significant in figure 4.7 is that the LTE class is assigned in class 0 in case of both TCP and SCTP audio traffic. that means the audio packets have the priority in the the buffer for every node and they should be transmitted as soon as possible. Also, in our simulation we have only one audio session between one mobile user and the server so the congestion between same level of priority does not occur in this case. Otherwise, the instantaneous delay will be worse especially for TCP.

\subsubsection{Video Traffic}

A simulation for video traffic was also run. It also had two handover times between the renewable and conventional energy base stations. The output results are displayed in Figure 4.8. In this case the video traffic is assigned to be video streaming (i.e. watching an MP4 file). The LTE class in this case is assigned to be class 1. This video file (akiyo_cif.yuv) as described in chapter three is $45.6 \mathrm{MB}$, but after converting it to an MP4 it becomes $630 \mathrm{~KB}$ (5160960 bits). To send this $5.16 \mathrm{M}$ bits file in ten seconds, a bit rate of $0.52 \mathrm{Mbps}$ is needed. This is consistent with the result of instantaneous throughput as demonstrated in Figure 4.9. In this figure the throughput in the case of SCTP saturates around 0.63 Mbps. Regarding the observed discontinuity at the time of 13.5 seconds, this it is because the video stream have finished (the video is ten seconds long) and it had been retransmitted 


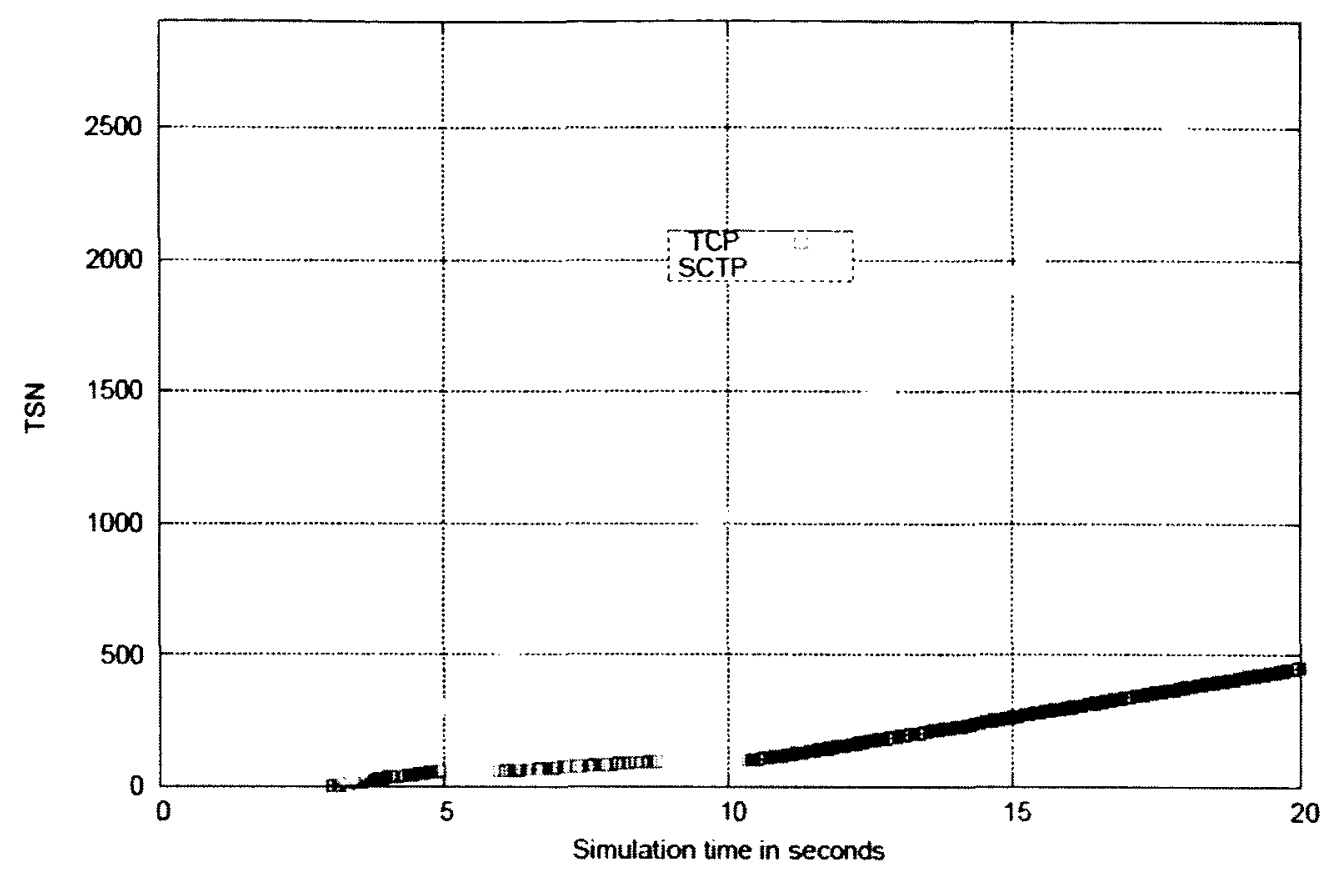

Figure 4.5: SCTP vs. TCP TSN behaviour for audio traffic

again. Also it is clear from the this figure that the throughput in the case of TCP saturates around $0.23 \mathrm{Mbps}$ which means some jam and delay are expected. More than ten seconds time is needed to transport this ten seconds length video stream. Finally the instantaneous delay is evaluated from the trace file as demonstrated in figure 4.10. As shown in this figure, the instantaneous delay in the case of SCTP is $0.25 \mathrm{sec}$ maximum. However, it is worse in the case of TCP where the delay jumps to more than one second in some packets and some other packets has more than 0.5 seconds delay. This high delay is too much for a video stream transmission. Based on our results, SCTP average delay per packet is much better than that of TCP for all traffic types we applied in the simulation. Figure 4.11 demonstrates a summary of the average delay per packet for FTP, audio and video traffic, which indicates the Superiority of SCTP. 


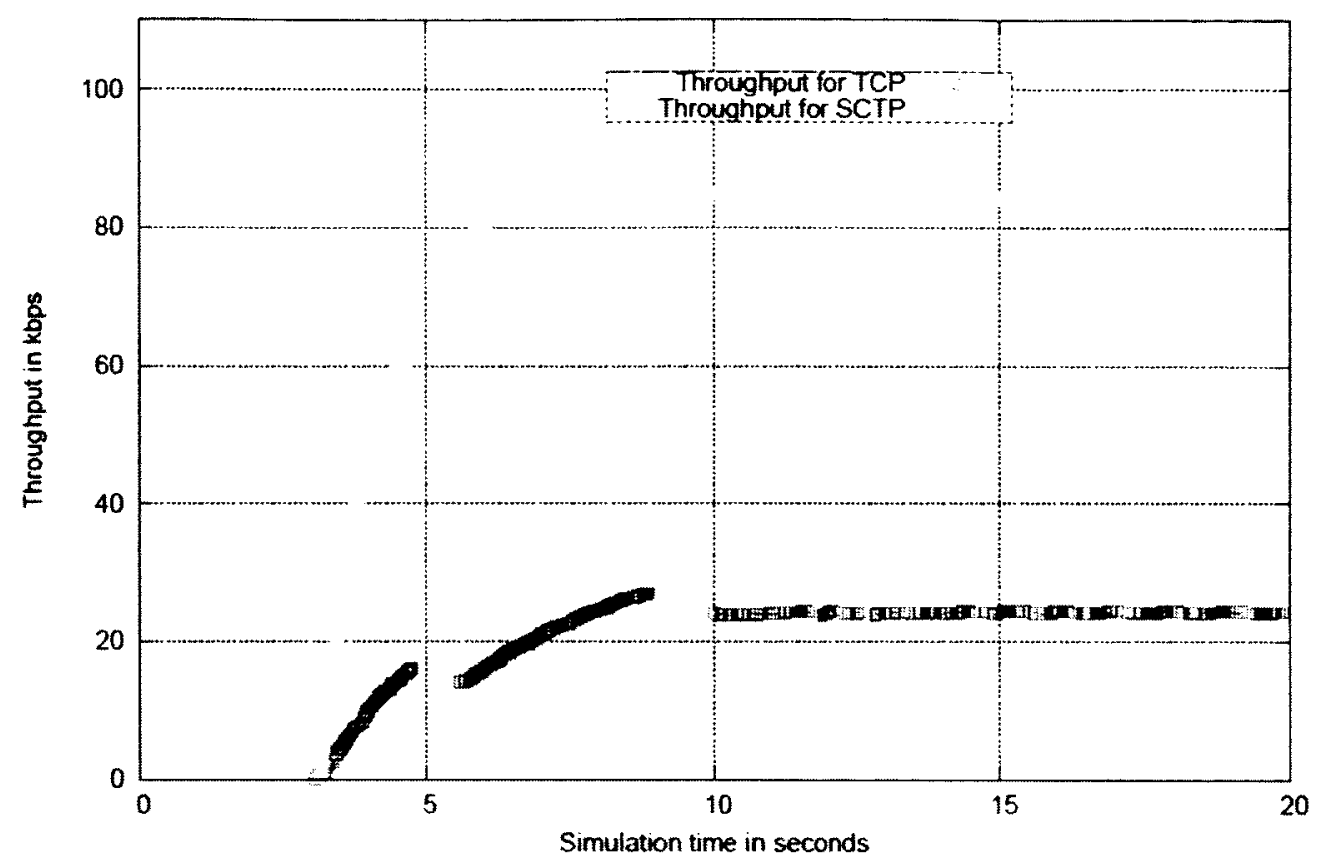

Figure 4.6: SCTP vs. TCP instantaneous throughput behaviour for audio traffic

\subsection{Summary}

In this Chapter we have provided a quick survey of the available simulation tools and why ns-2.33 was chosen for our purposes. The most significant reasons are the SCTP protocol with LTE models supportability and being very public, which is very important for academia. We have shown and explain the simulation outputs and results of our suggested solution Improved Green Cellular Technique by Employing SCTP Based Handover Mechanism using ns-2 as a simulation tool (version ns-2.33). In addition, we have discussed the required patches such as the LTE models to test the performance of TCP and SCTP protocols in carrying FTP, Audio and Video stream in the wire-cum-wireless network during the handover events. We have shown the capability, superiority and excellence of SCTP performance over TCP performance. Thus SCTP is high recommended to be the transport layer protocol in the green 


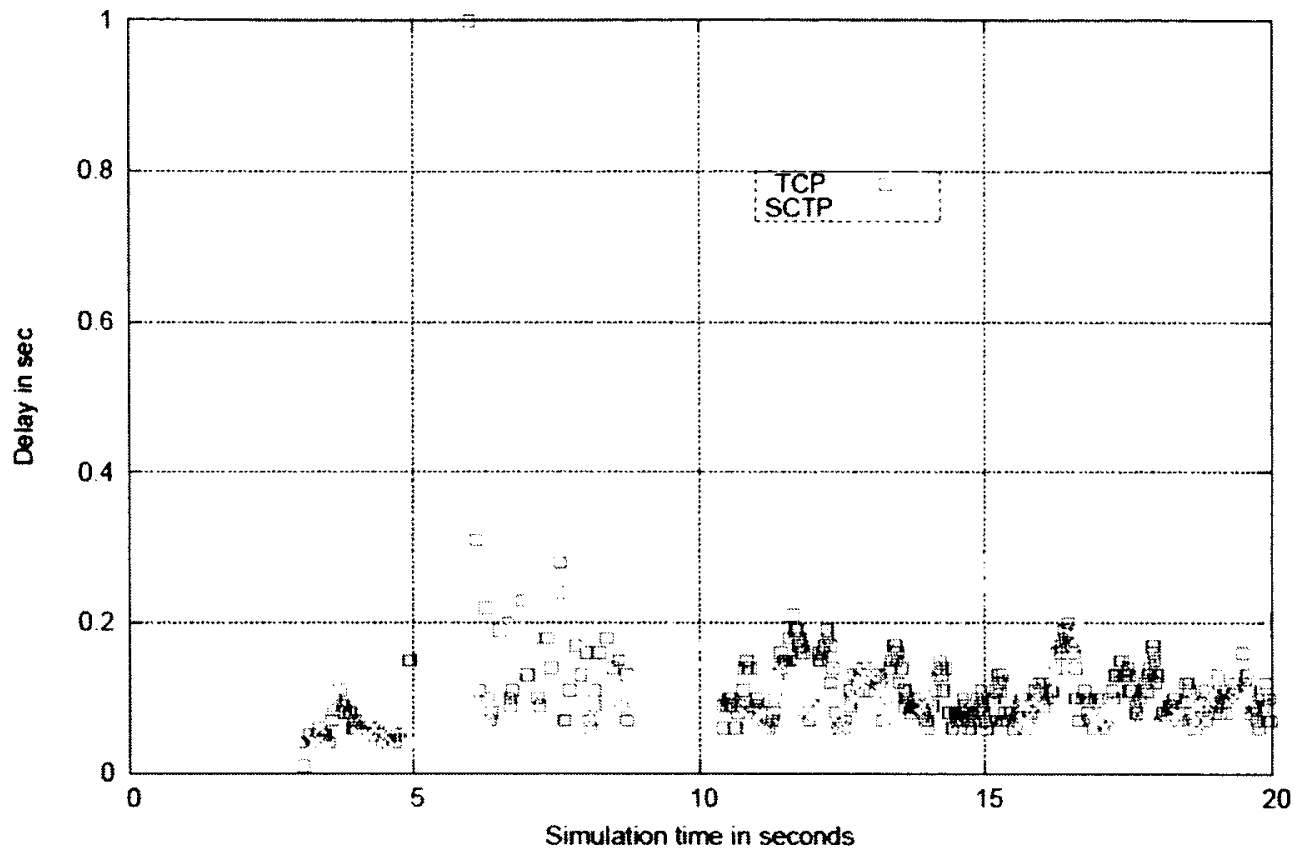

Figure 4.7: SCTP vs. TCP delay behaviour for audio traffic

wireless network.

The simulation consisted of sending FTP, audio, and video streams between two endpoints in a mixed network considering LTE models and under the conditions of using a renewable energy source to feed the wireless part of the network in conjunction with a traditional energy source. The simulation applied more than one handover between the access point fed by the renewable energy feeder to that fed by the traditional energy feeder as an analogy to what happens in the field when the renewable energy feeder goes down. The mobile user should be handed over to the access point fed by the traditional energy feeder temporarily until the renewable energy feeder is up again, and then the user should be handed back to save energy.

The comparison between SCTP and TCP involved three essential criteria: the TSN, the instantaneous throughput, and the instantaneous delay. SCTP was found 


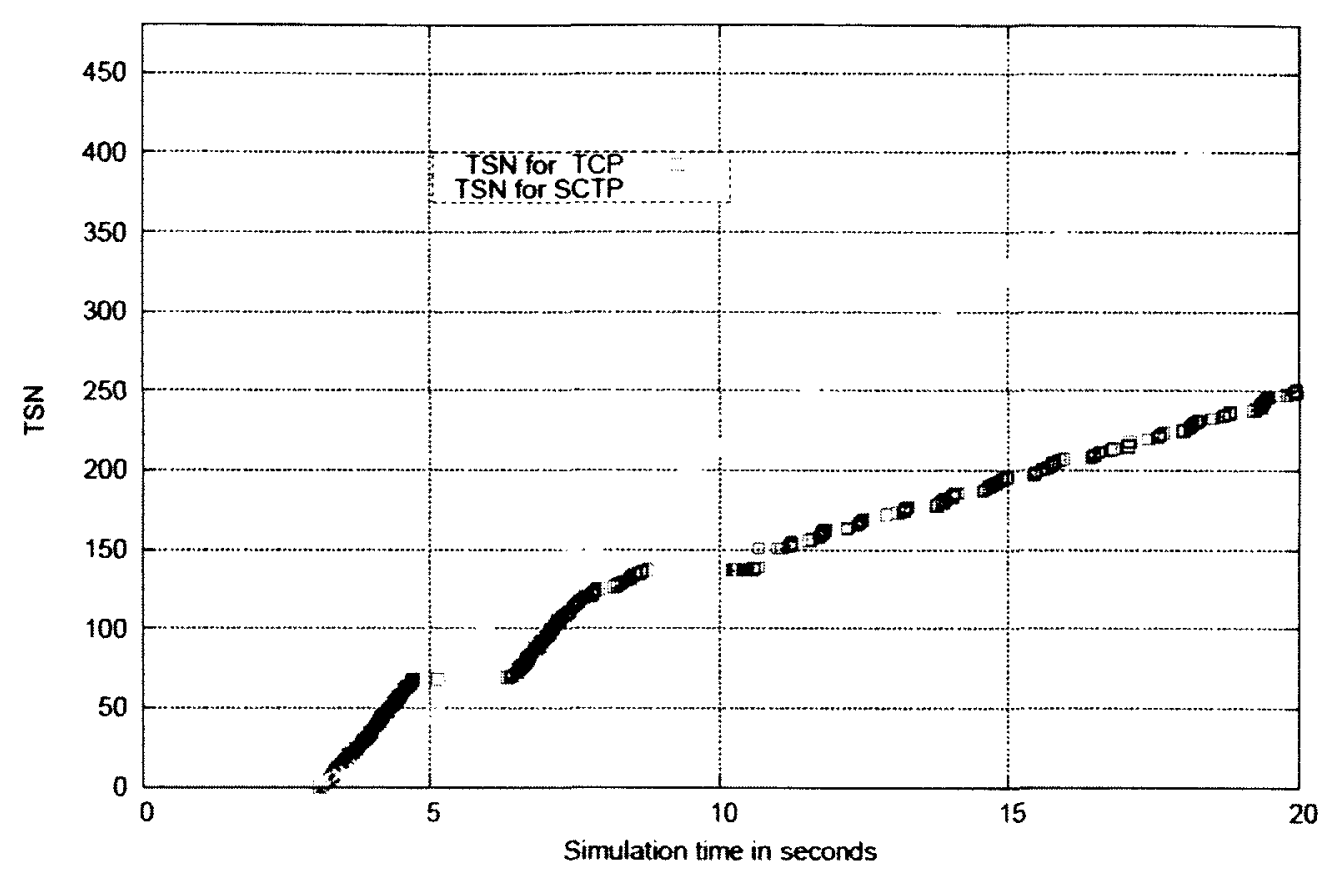

Figure 4.8: SCTP vs. TCP TSN behaviour for video traffic

to be capable in reaching more TSN than could TCP, especially in the video stream. This gives an indication that SCTP can send much more data without negative effects from the handover, unlike what happens in the case of TCP where the stream gets discontinued until an alternative connection is found. It is safe to say that the multi-homing and multi-streaming properties of SCTP play an essential role for the SCTP stream to be very stable. The multi-homing feature makes SCTP able to switch smoothly to an alternative path (other intermediate IP address) and the multi-streaming feature, in addition to being a message-oriented protocol, makes SCTP fragment the message into data chunks and send them independently. If there are any missing data chunks at the destination, this specific missing chuck will be re-sent, and it will be put in order when received at the destination. TCP has no multi-homing nor a multi-streaming feature, so it uses a strict order of delivery. Any 


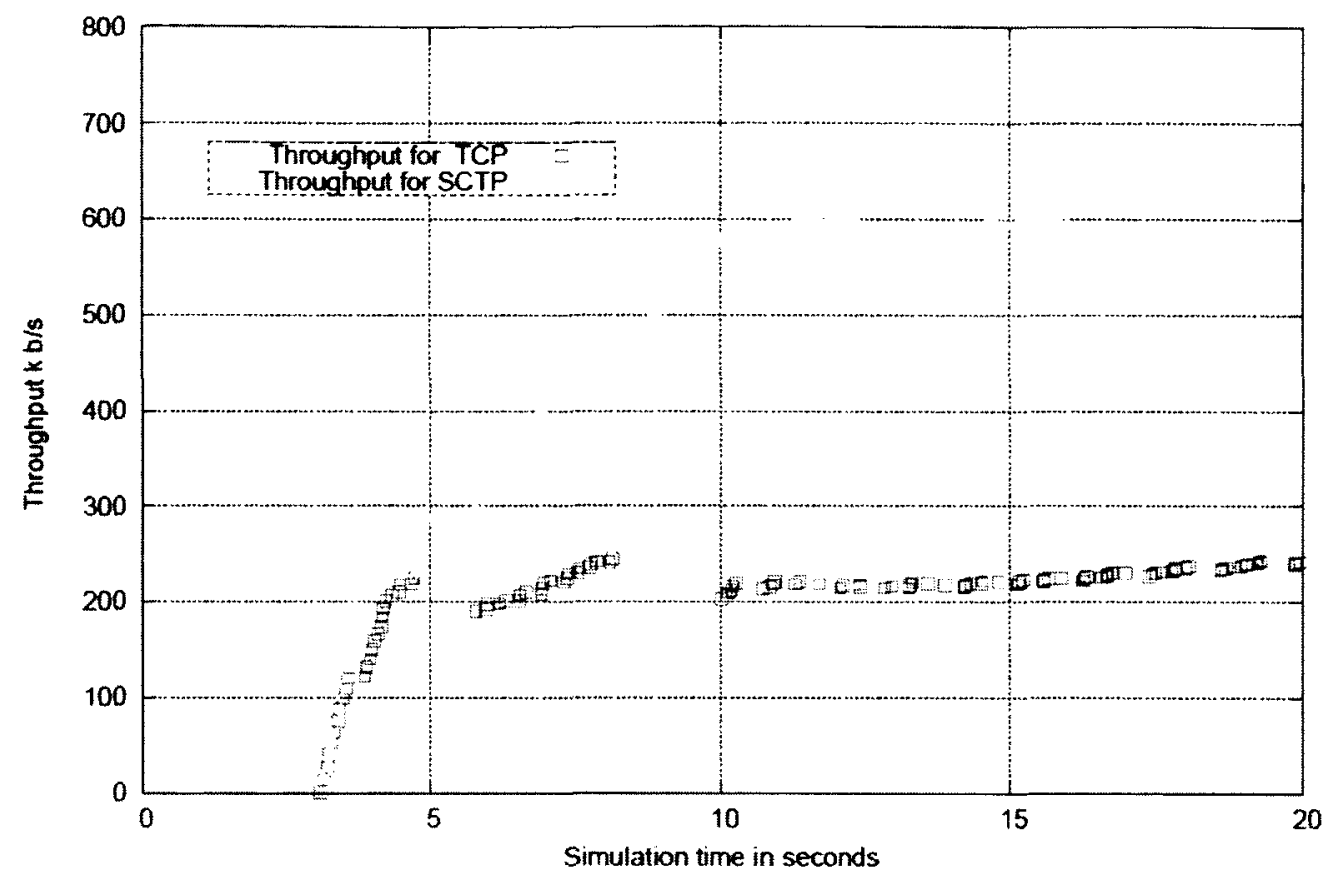

Figure 4.9: SCTP vs. TCP instantaneous throughput behaviour for video traffic

lost or unreceived packets will make the next ordered packets wait in the receiver buffer until this lost packet re-transmits, which makes these packets subject to delay or dropping out of the buffer. In addition, it is expected that the TSN behaviour in the case of TCP will be negatively affected during the handover. Finally, SCTP throughput increases dramatically without any remarkable effect from the handover, unlike TCP whose throughput decreases after the handover for the reason previously mentioned. 


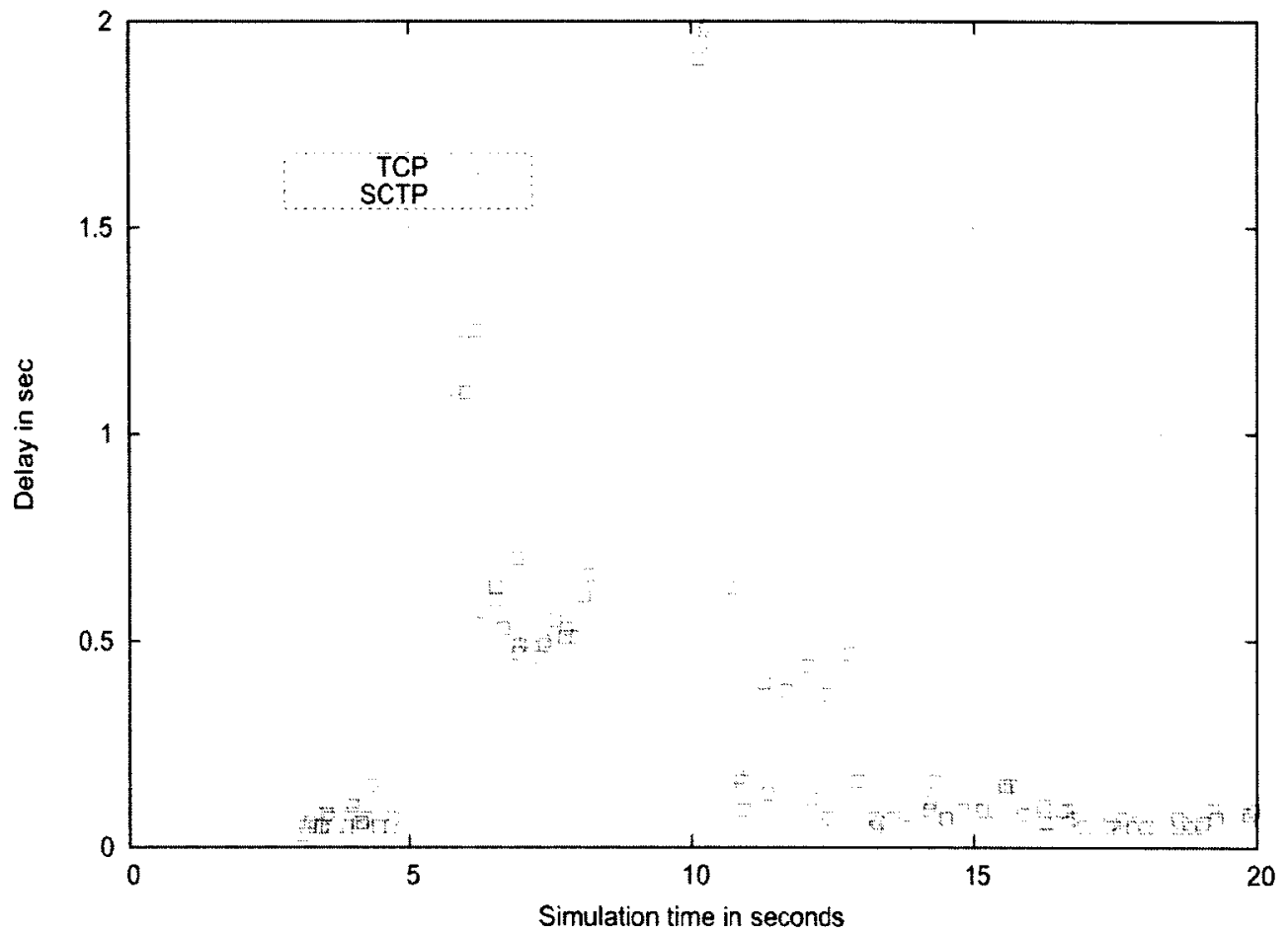

Figure 4.10: SCTP vs. TCP delay behaviour for video traffic 


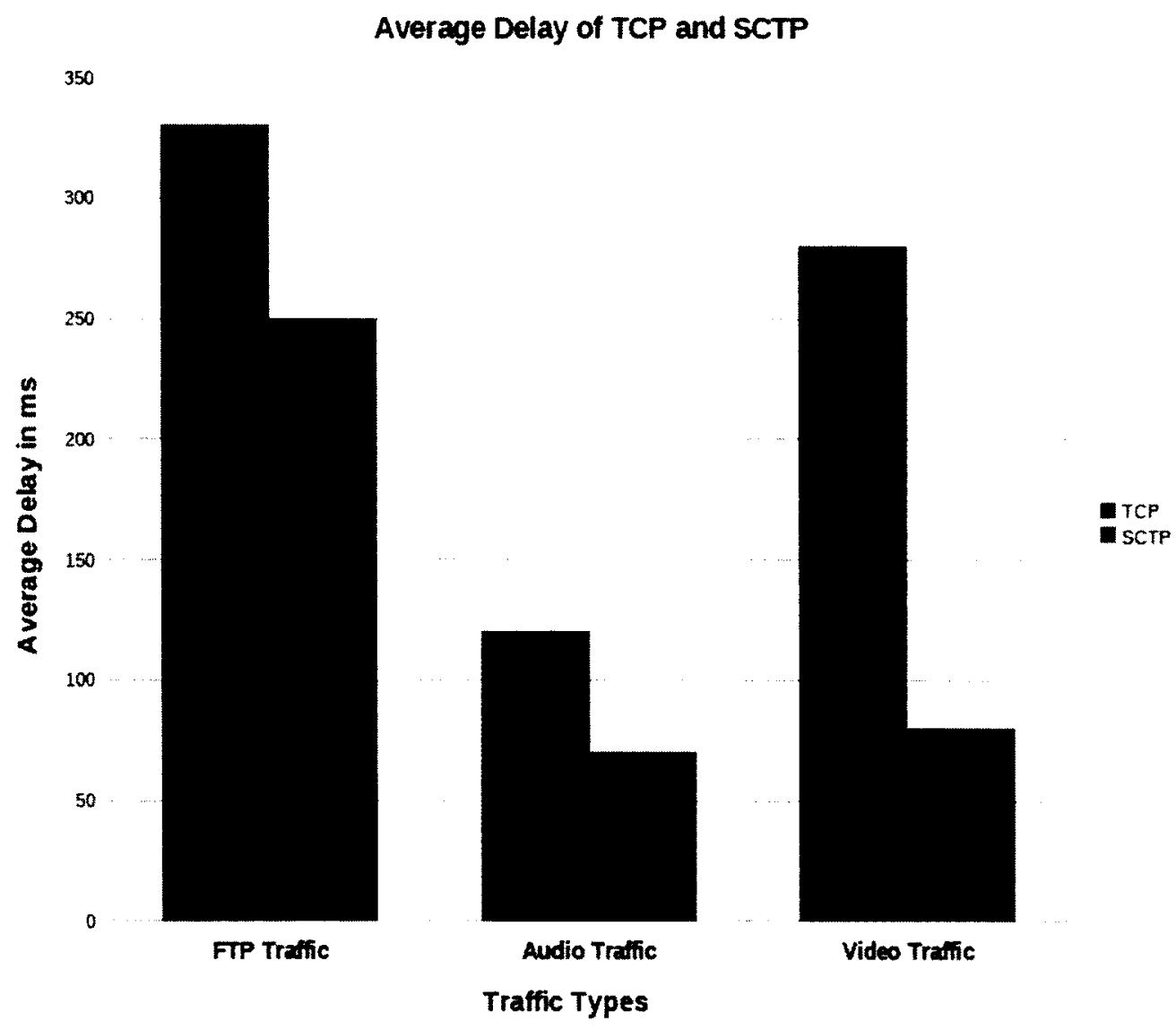

Figure 4.11: Average delay of TCP and SCTP for the simulated traffic types 


\section{Chapter 5}

\section{Conclusions and Future Work}

\subsection{Conclusions}

This thesis introduced a novel solution that employs the SCTP protocol to ensure a graceful handover between renewable and traditional energy fed base stations. The advantage of this solution is the ease of adoption by the interested parties. These include concerned commercial entities or residents, especially in remote areas, where they cannot be connected to traditional electricity. The overall goal is to reduce dependence on fossil fuels, which means lower gas emissions responsible for global warming, most notably carbon dioxide.

The proposed solution is a transport layer approach and it applies the end-to-end principle, where any change that can be done in the end of the system should to be done there. The network backbone (e.g. router network) remains the same when applying this proposed solution, which makes it easier to go adopt the SCTP proto-

col. Moreover, It is not just about reducing emissions, but it is also about increasing economic gains benefit for network operator by reducing operating expenses. While this proposed solution needs time to be deployed, it has been targeted towards access networks due to their large geographical area, increasing the probability that sustain- 
able energy will be used there. In Saudi Arabia (my country), for example, there are initiatives to adopt the main three sources of sustainable energy: wind energy, solar energy and sea waves energy.

Simulations have been conducted to show that the behaviour of the SCTP is impressive and touchable in the heterogeneous topology as compared to TCP. SCTP maintains and satisfies the quality of service for delay-sensitive streams such as audio or video streams. The packet lengths of SCTP and TCP were adjusted to be as close as possible to make a faire comparison. The importance of the reliability of the transmission protocol that carries and controls the video stream in the new generation of mobile network is not only for traditional entertainment video streams targeted for broadcasting (multi-casting or uni-casting) between peers in a network or between users and servers. In fact, new and very advanced computer applications that interact with human body movements or reactions, known as haptics applications, need this reliability as well. For example, a few seconds of video of a user facial expressions or fingers movements could be a password to log to a remote system server, and the user would use a mobile camera to do the $\log$ on procedure. In this case it is very important for a secure account to keep the connection smoothly without considerable delay or stream disturbance. This is available in the SCTP protocol even during handover instants.

It has been shown in this thesis that SCTP keeps the continuity of the data streams, audio streams and video streams. Due to the multi-homing capability, it shifts to an alternative energy path when the primary one goes down. Moreover, the multi-streaming property of SCTP makes the handover process graceful, or in other words, at the handover time some of the data chunks can go through the primary path and any dropped chunks due to an interrupted link will not affect the following data chunks sent through an alternative path. This is because SCTP segments can be 
reordered according to their TSN number, even if some higher ordered TSN arrives before a lower ordered TSN. This capability is not available in TCP. In the trace file that contains the time events trace of the simulation using ns-2, it was confirmed while applying SCTP, some higher ordered TSNs were received and acknowledged before some lower ordered TSNs.

\section{$5.2 \quad$ Future Work}

Our future work plan includes two dimensions: to expand the access network and to change the simulation tool. So far we have concentrated on micro position area for applying the SCTP based handover such as small cell. We are now interested in applying SCTP handover scenario on the scale of macrocells such as WIMAX (Worldwide Interoperability for Microwave Access) [62] to make the renewable energy utilization more practical and more efficient. In this case the number of mobile users will be much higher. In addition to that, the per user traffic demand is very huge. There is a new (on going) version of network simulator called network simulator version 3 (ns-3), and it is expected to replace the popular ns-2. Ns-3 last release is ns-3.13 which has been released in 2011 and came with enhanced features such as graphic user interface (GUI).

System architecture Evolution (SAE) is considered to be the backbone network architecture (core network) of LTE (Long Term Evolution) wireless standard. The most important part of SAE architecture is the evolved packet core (EPC), which cares about Mobility Management Entity, the Serving Gateway and the Packet Data Network (PDN) gateway.

The LTE-EPC network simulator (LENA) is a newly released open-source simulator [64]. This simulator is based on ns-3 network simulator, which means all the transport protocols supported by ns-3 are included (e.g. TCP and UDP) or can be 
included soon (e.g. SCTP). Ns-3 currently supports TCP and UDP protocols in the transport layer but does not handle SCTP protocol because SCTP modules are not part of ns-3 package yet. Once the ns-3 developers group add SCTP to ns-3, it would be possible to use SCTP with LENA after doing the required modifications on the classifier to decode the SCTP header. We are planning to go through that and use LENA simulator applying SCTP as transport protocol because LENA simulator provides platforms of LTE vendors (LTE femto and LTE macro vendors). The LTE, as the access part of the evolved packet system (EPS), will maintain the requirements for the modern access network, such as high spectral efficiency, high data rate and frequency flexibility. Our plane is to create a heterogeneous topology consisting of an LTE access network (base stations and evolved nodes) and WIMAX access network, in additional to the core network (EPC), which is capable to work with these different access technologies. This topology can be simulated by employing LENA over ns-3 to cover WIMAX standards or any other access technology not developed by 3GPP (3rd Generation Partnership Project) such as WIFI. The users will be handed over from renewable energy fed base stations (LTE femtocells base station or WIMAX base station) and the conventional energy fed base station in this heterogeneous topology, even if the two peers are not in the same cell to check the traffic quality of service $(\mathrm{QOS})$.

In addition to the MAC and transport layer, our plan is to consider multimedia traffic streams, especially video. Since the QoS parameters of the stream during the handover instant is very important, we will apply the QoS in the application layer level, not only in the transport layer. Our plan includes subjective metrics to assess the quality of the video stream, such as mean opinion score (MOS) and peak signal noise ratio (PSNR). It is very impressive to send a watermarked media (mainly video) and check the affection due to several times of SCTP based handover that are 
expected between the renewable energy fed base station to conventional energy fed base station.

Finally, SCTP was originally designed to cover the signalling between the trunk gateways of circuit switch based networks to new IP-based networks (next generation network). In the IP-based network, session initiation protocol (SIP) is employed to control communication sessions over IP. Using SCTP as the transport protocol for SIP in heterogeneous network is a considerable idea for our future plans. 


\section{References}

[1] M. Akhtar, S. Simonovic, J. Wibe J. MacGee, and J. Davies, An Integrated System Dynamics Model for Analyzing Behaviour of the Social-Energy-EconomicClimatic System, Department of Civil and Environmental Engineering, The University of Western Ontario, ISBN (On-line) 978-0-7714-2897-5, August 2011.

[2] P. Asmus, and B. Ziskin, "Framing the Global Warming Policy Debate", Environmental Law and Policy Journal, vol. 14, pages 37-45, May 1990.

[3] J. Conti, U. S. EIA, Washington, D. C. , Rep. DOEIEIA-0573(2008), Emissions of Greenhouse Gases in the United States 2008, May 2008.

[4] Gartner, Inc., Gartner Highlights Key Predictions for IT Organizations and Users in 2010 and Beyond, www.gartner.com, last check on May 2012.

[5] R. Horak, Telecommunication and Data Communication Handbook, John Wiley and Sons, ISBN 978-0-470-04141-3, 1st edition 2007.

[6] T. Chen, H. Zhang, Z. Zhao and X. Chen, "Towards green wireless access networks," in Proc. of the International ICST Conference, Communications and Networking in China (CHINACOM.) vol. 13, no. 4, pages 1-6, August 2010. 
[7] M. Wu, and M. Peng, Developing a Tool to Estimate Water Use in Electric Power Generation in the United States. U. S. EIA, Washington, D. C. , Rep. DOEIEIA-0573(2008), Argonne National Laboratory, December 2010.

[8] M. Bollen, and F. Hassan, Integration of Distributed Generation in the Power System, Wiley-IEEE Press \& eBooks, ISBN 9781118029039, Chapter:2, pages 31-222, 1st edition 2011.

[9] B. Leiba., P. Natarajan, F. Baker, P. Amer and J. Leighton, A white paper in, "SCTP : Why, What, and How," in IEEE Internet computing, vol. 13, no. 5, pages 81-85, September/October 2009.

[10] A. Amanna, J. Reed, T. Bose and T. Newman, A white paper in, "Metrics and Measurement Technologies for Green Communications," National Institute of Standards and Technology Innovation Program, March 2009.

[11] Z. Hasan, H. Boostanimehr and V. Bhargava, "Green Cellular Networks: A Survey, Some Research Issues and Challenges," in Proc. IEEE Communications surveys and Tutorials, vol. 13, no. 4, pages 524-540, Fourth Quarter 2011.

[12] J. Twidell, A white paper in, "Renewable Energy: Implementation and Benifits," in Proc. IEE 2nd international Conference on Advances in Power System Control, Operation and Management, pages 418 -424, December. 1993, Hong Kong.

[13] N. Sriram, and M. Shahidehpour, "Renewable Biomass Energy," in Proc. IEEE Power Engineering Society General Meeting, vol. 1, pages 612-617, June 2005.

[14] S. Payyala, and T. Green, "An Estimation of Economic Benefit Values of DG," in Proc. IEEE Power Engineering Society General Meeting, pages 1-8, June 2007 . 
[15] C. Dragu, T. Sels, and R. Belmans, "Small hydro power-State of the art and applications," in Proc. of International Conference on Power Generation and Sustainable Development (AIM), Lige, Belgium, pages 265-270, October 2001.

[16] Y. Ma, H. Jiang, and B. Wang, "Dynamics of the Carbon Storage of Terrestrial Ecosystems in Geological Spatial and Temporal Scales," in Proc. of the 19th International Conference on Geoinformatics, pages 1-4, June 2011.

[17] G. Liu, and Y. Zhuang, "Application and Construction of Geothermal Pump Technology in Energy Efficiency," in Proc. of Power and Energy Engineering Conference, pages 1-4, March 2009.

[18] S. Rahman, A white paper in, "Green power: what is it and where can we find it?," IEEE Power and Energy Magazine, pages 30-37, January/February 2003.

[19] V. Quaschning, Renewable Energy and Climate Change, John Wiley and Sons Ltd Chichester ISBN 978-0-470-74707-0, pages 210 - 222 1st edition 2010.

[20] M. Birney, A white paper in, "Small Wind Potential for Personal Energy Independence," Available on line http:/www.cecsb.org/image/stories/what-wedo/small_wind_white_Paper_v4.pdf, February 2010, last visited on May 15th 2012.

[21] S. Pal, "Wind Energy - An Innovative Solution to Global Warming," in Proc. of the 1st International Conference on the Developments in Renewable Energy Technology (ICDRET), pages 1-3, December 2009.

[22] L. Merugula, V. Khanna, and B. Bakshi, "Comparative Life Cycle Assessment: Reinforcing Wind Turbine Blades with Carbon Nanofibers," in Proc. IEEE International Symposium on Sustainable Systems and Technology (ISSST), pages 1-6, May 2010. 
[23] R. Brenden, W. Hallaj, G. Subramanian and S. Katoch, "Wind Energy Roadmap," in Proc. of Portland International Conference on Management of Engineering and Technology (PICMENT 2009), pages 2548-2562, August 2009.

[24] U.S. Energy Information Administration, Rnewable Wind, Available in http://www.eia.doe.gov/kids/energyfacts/sources/renewable/wind.html, last visited on May 15th 2012.

[25] M. Lydia and S. Kumar, "A Comprehensive Overview on Wind Power Forecasting," in Proc. of the IPEC 2010 Conference, Pages 268-273, October 2010.

[26] F. Yu, P. Zhang, W. Xiao and P. Choudhury, "Communication Systems for Grid Integration of Renewable Energy Resources," IEEE Network, vol. 25, no. 5, pages 22 -29, September/October 2011.

[27] A. Tanenbaum, Computer Networks, Prentice-Hall International, 4th edition, 2002.

[28] R. Stewart, Q. Xie, K. Morneault, C. Sharp, H. Schwarzbauer, T. Taylor, I. Rytina, M. Kalla, L. Zhang, and V. Paxson, RFC2960 Stream Control Transmission Protocol, October 2000.

[29] R. Stewart, RFC4960 Stream Control Transmission Protocol, September 2007.

[30] J. Postel, RFC 793 Transmission Control Protocol, September 1981.

[31] J. Postel, RFC 768 User Datagram Protocol, August 1980.

[32] R. Stewart and C. Metz, A white paper in, "SCTP New Transport Protocol for TCP/IP," IEEE Internet Computing, vol. 5, no. 6, pages 64-69, November/December 2001. 
[33] D. Pacifico., M. Pacifico, C. Fischione, H. Hjalrmasson and K. Johnsson, "Improving TCP Performance During the Intra LTE Handover," in Proc. IEEE Global Telecommunications Conference (GLOBECOM), pages 1-8, November 2009 .

[34] C. Xu, E. Fallon, Y. Qiao, G.-M. Muntean, X. Li and A. Hanley, "Analysis of Real-time Multimedia Transmission over PR-SCTP with Failover Detection Delay and Reliability Level Differential," in Proc. of International Conference on Communication Software and Networks (ICCSN 09), pages 768-772, February 2009 .

[35] K. Lee, S. Nam, and B. Mun, "SCTP Efficient Flow Control During Handover," in Proc. IEEE Wireless Communication and Networking Conference (WCNC 2006), pages 69-73, April 2006.

[36] Alcatel-Lucent, Alternative Energy Program, Available in http://www.alcatellucent.com/alternative-energy/, last visited on September 15th 2012.

[37] B. Wang, Network Simulator NS2, Available in http://www.cse.msu.edu/ wangbol/ns2, last visited on October 2011.

[38] A. Chung, Traffic Engineering for Multi-homed Mobile Networks, PhD Thesis, The University of New South Wales, School of Computer Science and Engineering, Sydney, Australia, May 2007.

[39] J. Yan, L. Zhao and J. Li "A Prediction-Based Handover Trigger Time Selection Strategy in Varying Network Overlapping Environment," inProc. IEEE Vehicular Technology Conference (VTC Fall), pages 1-5. September 2011. 
[40] D. Negru. T. Ahmed and A. Mehaoua "Adaptive audio-video streaming solution over IP mobile environments," inProc. IEEE Wireless Communications and Networking Conference (WCNC 2006), vol.4, pages 2156-2161, April 2006.

[41] S. Nor, S. Hassan and O. Ghazali "'Friendliness of DCCP towards TCP over large delay link networks," inProc. of Education Technology and Computer Conference (ICETC), vol.5, pages 286-291, June 2010.

[42] Arizona State University, YUV Video Sequences, Available in http://trace.eas.asu.edu/yuv/index.html, last visited on March 15th 2012.

[43] Wikipedia, $\quad Y U V, 14$ September 2012, Available in http://en.wikipedia.org/wiki/yuv, last visited on March 15th 2012.

[44] Wikipedia, MPEG-4 Part 14, 2 September 2012, Available in http://en.wikipedia.org/wiki/Mp4, last visited on March 15th 2012.

[45] K. Fall and K. Varadhan, The ns Manual (formerly ns Notes and Documentation), VINT Project, November 2011.

[46] J. Liu, A white paper in, "Parallel Discrete-Event Simulation," Florida International University, School of Computing and Information Sciences, February 2009.

[47] J. Lessmann, P. Janacik, L. Lachev and D. Orfanus, "Comparative Study of Wireless Network Simulators," in Proc. of the Seventh International Conference on Networking (ICN 2008), pages 517-523, April 2008.

[48] J. Pan, "A Survey of Network Simulation Tools: Current Status and Future Developments:" Available in http://www.cse.wustl.edu/ jain/cse56708/ftp/simtools/index.html, November 2008, last visited on May 15th 2012. 
[49] S. Mehta, N. Sulatan, H.Kabir, N.Ullah and K. Kwak, "Network and System Simulation Tools for Next Generation Networks: A Case Study," August 2010.

[50] E. Egea-Lopez, J. Vales-Alonso, A. Martinez-Sala, P. Pavon-Marino and J. Garcia-Haro, "Simulation Scalability Issues in Wireless Sensor Networks," IEEE Communications Magazine, vol. 44, pages 64-73, July 2006.

[51] K. Wehrle and M. Gne, Modeling and Tools for Network Simulation, Springer ISBN:978-3-642-12330-6, chapter:17, pages 385-395, 2006.

[52] T. Henderson Improving Simulation Credibility Through Open Source Simulations,, Available in tomh.org/talks/simutools08-keynote-final.pdf, March 2008, last visited on May 15th 2012.

[53] T. Issariyakul and E. Hossain, Introduction to Network Simulator NS2, Springer ISBN:978-0-387-71760-9, 2009.

[54] Information Sciences Institute ISI, Network Simulator 2, Available in http://nsnam.isi.edu/nsnam/index.php/Main_Page, last visited on February 1st 2012.

[55] Q. Qiu, J. Chen, L. Ping, Q. Zhang and X. Pan "LTE/SAE Model and its Implementation in NS 2," in Proc. of the 5th International Conference on Mobile Ad-hoc and Sensor Networks, pages 299-303, December 2009.

[56] J. Klaue, B. Rathke and A. Wolisz, "EvalVid - A Framework for Video Transmission and Quality Evaluation," inProc. of the 13th International Conference on Modelling Technique and Tools for Computer Performance Evaluation, September 2003 . 
[57] C. Ke, How to evaluate H.263/H.264/MPEG4 video transmission using the NS2 simulator, Available in http://140.116.164.80/ smallko/ns2/myevalvid2.htm, last visited on December 1st 2011.

[58] M. Bateman and S. Bhatti, "TCP Testing: How Well Does ns2 Match Reality?," inProc. of the 24th IEEE International Conference on Advanced Information Networking and Applications (AINA), pages 276-284, April 2010.

[59] M. Tahiliani, AWK Script for NSE, Available in http://mohittahiliani.blogspot.ca/2009/12/awk-script-for-ns2.html, last visited on December 1st 2011.

[60] B. Barnett $A W K$, Available in http://www.grymoire.com/Unix/Awk.html, last visited on October 1st 2011.

[61] T. Williams and C. Kelley gnuplot 4.6, An Interactive Plotting Program, Available in http://www.gnuplot.info/docs_4.6/gnuplot.pdf, last visited on December 15 th 2011.

[62] WiMax.com Broadband Solutions, Inc., What is WiMAX?, Available in http://www.wimax.com, visited on May 15th 2012.

[63] N. Baldo, M. Miozzo, M. Requena-Esto, and J. Nin-Guerrero, "An Open Source Product-Oriented Network Simulator based on ns-3," In Proc. of the 14th ACM International Conference on Modeling, Analysis and Simulation of Wireless and Mobile Systems (MSWIM 2011), pages 293-298, November 2011.

[64] Centre Tecnolgic de Telecomunicacions de Catalunya (CTTC), LTE-EPC Network Simulator (LENA), Available in http://iptechwiki.cttc.es/LTEEPC_Network_Simulator_(LENA), last visited on May 1st 2012. 


\section{Appendix A}

\section{Simulation Scripts}

The ns-2 simulation script for sending FTP, audio and video traffic over both TCP and SCTP in our proposed scheme is described as follows.

\section{A.1 FTP and Audio Traffic via TCP and SCTP}

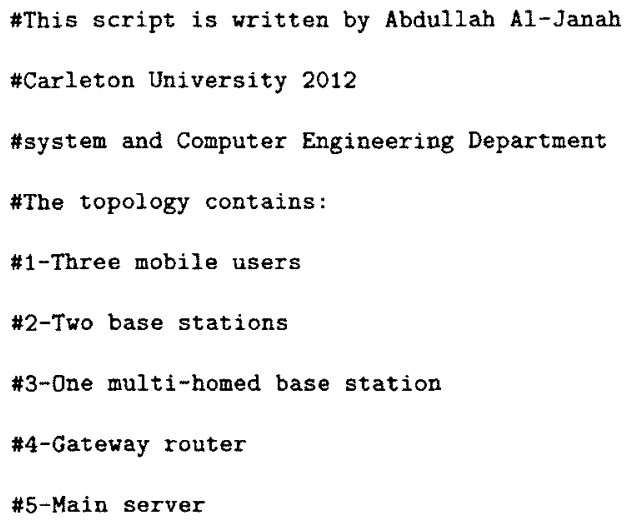




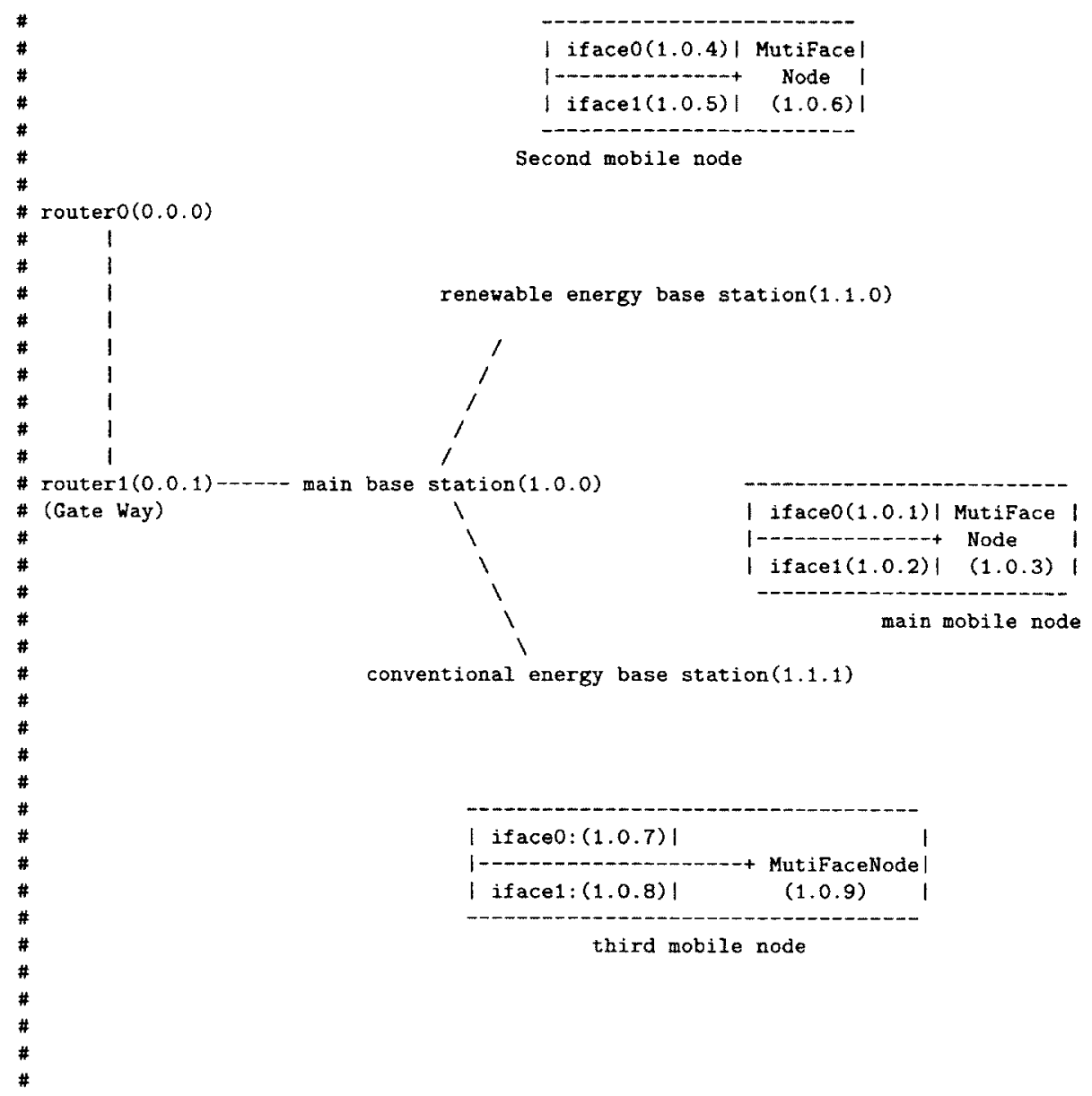

\#To set the simulation parameters for wire and wireless

\begin{tabular}{|c|c|c|c|}
\hline set & opt (chan) & Channel/WirelessChannel & ;\# channel type \\
\hline set & opt (prop) & Propagation/TwoRayGround & ;\# radio-propagation model \\
\hline set & opt (netif) & Phy/WirelessPhy & ; network interface type \\
\hline set & opt (mac) & $\mathrm{Mac} / 802_{m} 11$ & ;\# MAC type \\
\hline set & opt (ifq) & Queue/DropTail/Priqueue & ;\# interface queue type \\
\hline set & opt (11) & LL & ; \# link layer type \\
\hline set & opt (ant) & Antenna/OmniAntenna & ;\# antenna model \\
\hline set & opt (ifqlen) & 50 & ; \# max packet in ifq \\
\hline set & opt (an) & 3 & ; number of mobilenodes \\
\hline set & opt (adhocRouting) & DSDV & ;\# routing protocol \\
\hline set & opt (cp) & "n" & ; connection pattern file \\
\hline $\begin{array}{l}\text { set } \\
\text { \# us }\end{array}$ & $\begin{array}{l}\text { opt (sc) } \\
\text { sed }\end{array}$ & ility/scene/scen-3-test" & ; node movement file. \%ot \\
\hline set & opt (err) & MarkovErrorModel & \\
\hline set & $\operatorname{opt}(x)$ & & $x$ coordinate of topology \\
\hline
\end{tabular}




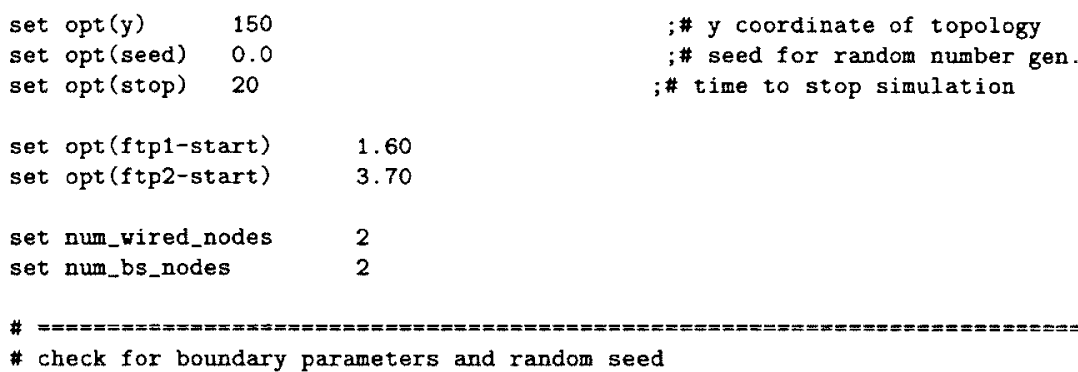




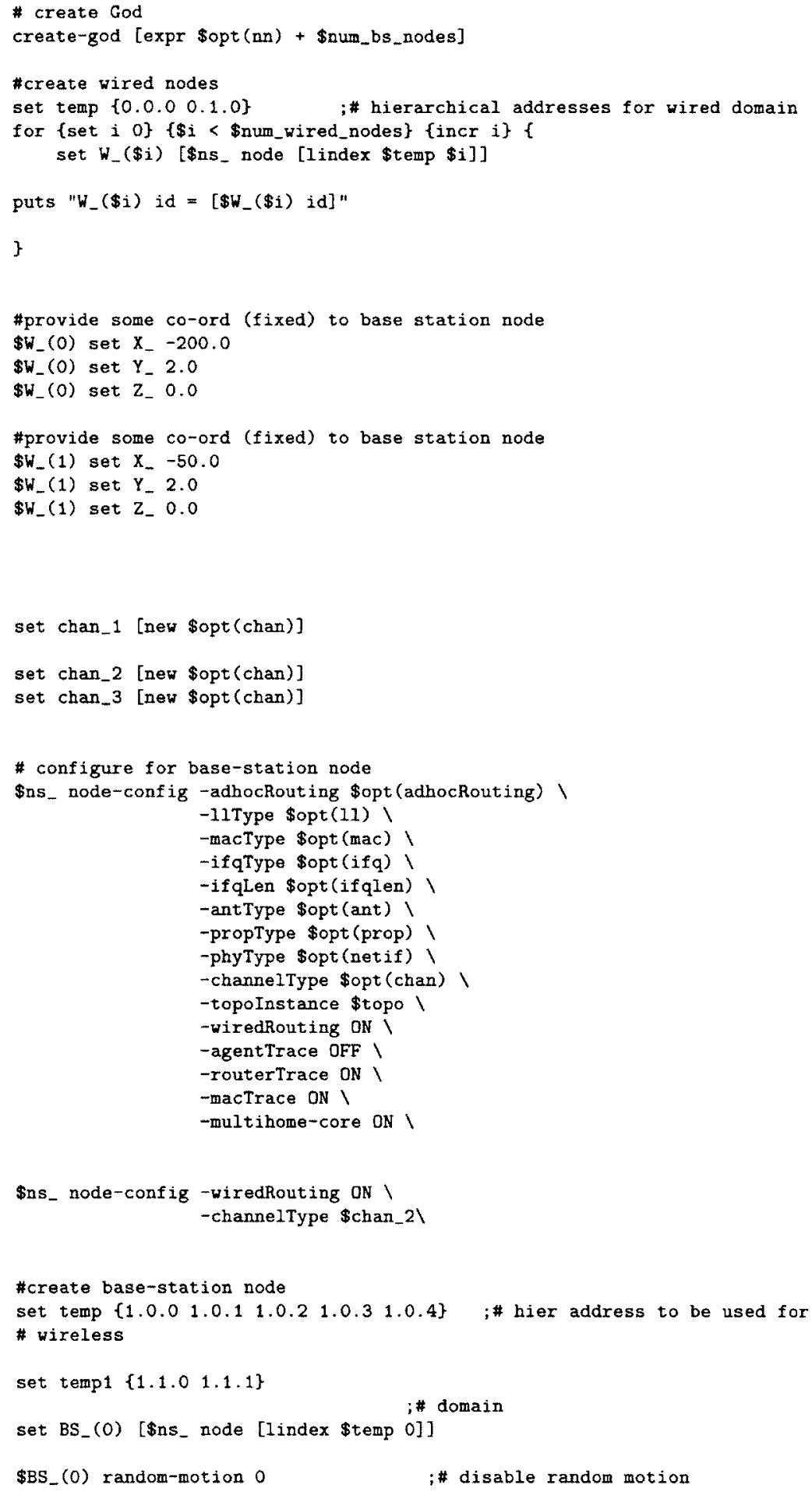




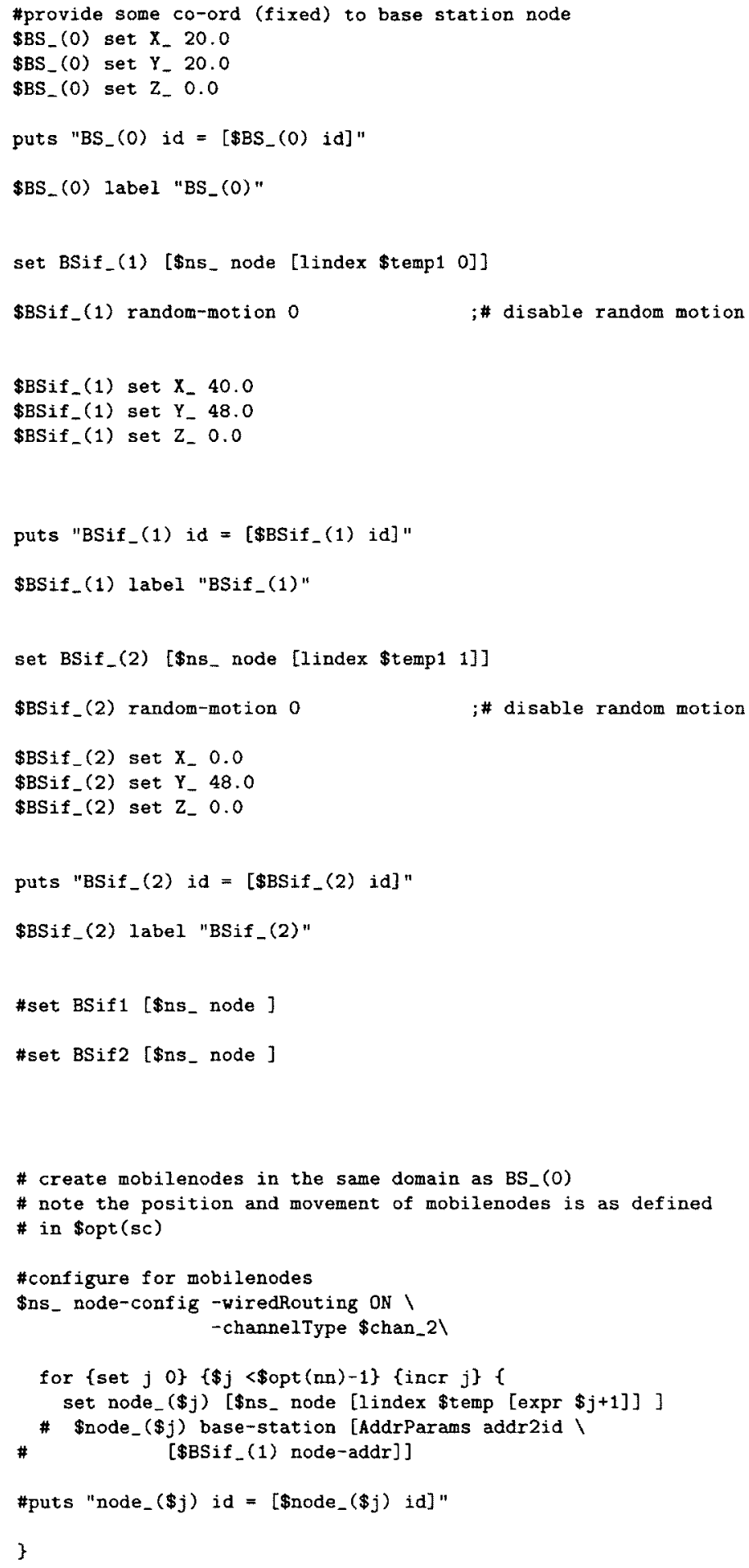


\$node_(0) random-motion 0

; disable random motion

\#\$node_(2) base-station [AddrParams addr2id [\$BS_(0) node-addr]]

\$node_(0) set $X_{-} 140.0$

\$node_(0) set $Y_{-} 100.0$

\$node_(0) set $Z_{-} 0.0$

\$node_(1) random-motion 0

; disable random motion

\#\$node_(2) base-station [AddrParams addr2id [\$BS_(0) node-addr]]

\$node_(1) set $X_{-} 140.0$

\$node_(1) set $Y_{-} 90.0$

\$node_(1) set $Z_{-} 0.0$

\$node_(1) label "node_(1)"

\$node_(0) label "node_(0)"

\$node_(0) base-station [AddrParams addr2id

[\$BSif _(1) node-addr]]

\$node_(1) base-station [AddrParams addr2id

[\$BSif_(2) node-addr]]

\# set Node_(5) [\$ns_ node 1.0 .4$]$

\# set routero [\$ns node 3.0 .0 ]

\#puts "node_(2) id $=[\$$ node $(2)$ id $] "$

\$ns_ node-config -channelType \$chan_2\

set node_(2) [\$ns_ node 1.0 .3 ]

\$node_(2) random-motion 0

disable random motion

\$node_(2) base-station [AddrParams addr2id [\$node_(0) node-addr]]

$\$$ node_(2) set $X_{-} 150.5$

\$node (2) set $Y$ 95.0

\$node_(2) set $Z_{-} 0.0$

set multihome-core \$node_(2)

\#[\$node (2) set mac (0)] set-channel 3

\#[\$node_(2) set mac_(0)] set-channel 3

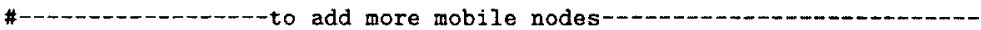

set temp2 $\left\{\begin{array}{llllllllllllll}1.0 .0 & 1.0 .1 & 1.0 .2 & 1.0 .3 & 1.0 .4 & 1.0 .5 & 1.0 .6 & 1.0 .7 & 1.0 .8 & 1.0 .9\end{array}\right.$

for $\{$ set $j 3\}\{\$ j<\$ o p t(n n)+2\}$ incr $j\}\{$

set node_(\$j) [\$ns_ node [lindex \$temp2 [expr $\$ j+1]]$

\# Snode_( $\$ j)$ base-station [AddrParams addr2id । [\$BSif_(1) node-addr]]

puts "node $(\$ j)$ id $=[\$$ node $(\$ j) i d] "$ 
Snode_(3) label "node_(3)"

\$node_(4) label "node_(4)"

\$node_(3) random-motion 0 ; disable random motion

\#\$node_(2) base-station [AddrParams addr2id [\$BS_(0) node-addr]]

\$node_(3) set $X_{-}-140.0$

\$node_(3) set $Y_{-} 100.0$

Snode_(3) set $Z_{-} 0.0$

\$node... (4) random-motion 0

$$
\text { ; disable random motion }
$$

\#\$node_(2) base-station [AddrParams addr2id [\$BS_(0) node-addr]]

\$node_(4) set $X_{-}-140.0$

\$node_(4) set $Y_{-} 90.0$

\$node_(4) set $Z_{-} 0.0$

\#\$node_(3) base-station [AddrParams addr2id

[\$BSif _(1) node-addr]]

\$node_(4) base-station [AddrParams addr2id

[\$BSif_(2) node-addr]]

\$ns_ node-config -hannelType \$chan_2

set node_(5) [\$ns_ node 1.0 .6$]$

\$node_(5) random-motion 0

disable random motion

\$node_(5) base-station [AddrParams addr2id [\$node_(4) node-addr]]

\#\$node_(0) base-station [AddrParams addr2id

[\$BSif_(1) node-addr]]

\$node_(5) set $X_{-}-150.5$

$\$$ node_(5) set $Y_{-} 95.0$

\$node_(5) set $z_{-} 0.0$

\#\$node_(5) base-station [AddrParams addr2id [\$node_(0) node-addr]]

set multihome-core \$node_(5)

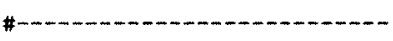

\#--

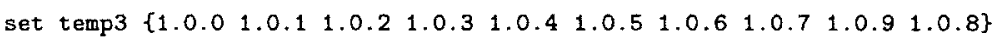

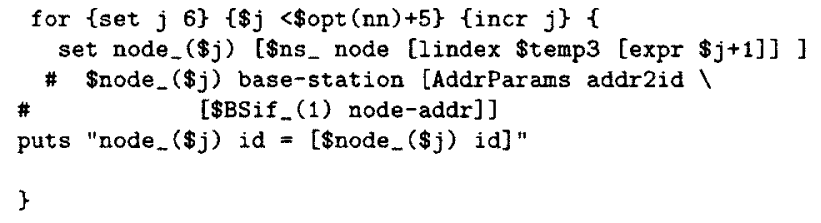


\$node_(6) set $X_{-}-100.0$
\$node_(6) set $Y_{-} 45.0$

\$node_(6) set $Z_{-} 0.0$

\$node_(7) random-motion 0

; disable randon motion

\#\$node_(2) base-station [AddrParams addr2id

[\$BS_(0) node-addr]]

\$node_(7) set $X_{-}-100.0$

$\$ n o d e_{-}(7)$ set $Y_{-} 35.0$

$\$$ node_(7) set $Z_{-} 0.0$

\$ns_ node-config -hannelType \$chan_2

-multihome-core ON

set node_(8) [\$ns_ node 1.0 .8 ]

\$node_(8) random-motion 0

\# disable random motion

\$node_( 8 ) base-station [AddrParams addr2id

[\$node_(6) node-addr]]

\# \$node_(0) base-station [AddrParams addr2id

[\$BSif_(1) node-addr]]

\$node_(8) set $x_{-}-110.0$

$\$$ node_(8) set $Y_{-} 40.0$

\$node_(8) set $Z_{-} 0.0$

\# \$node_( 8 ) base-station [AddrParams addr2id

set multihome-core \$node_(8)

[\$node_(0) node-addr]

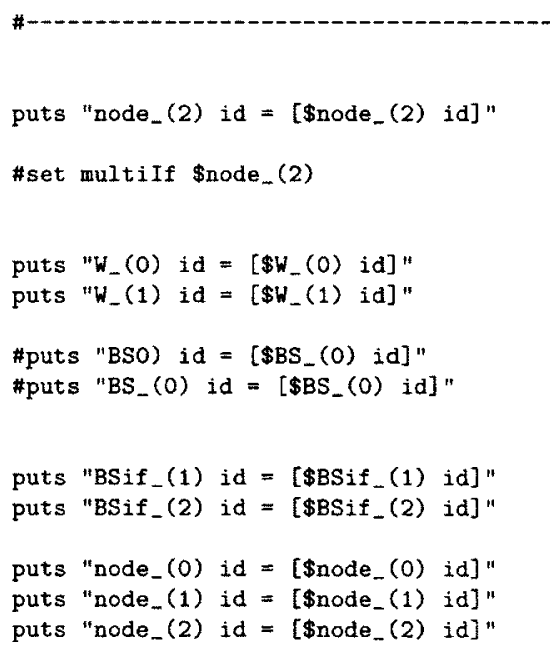




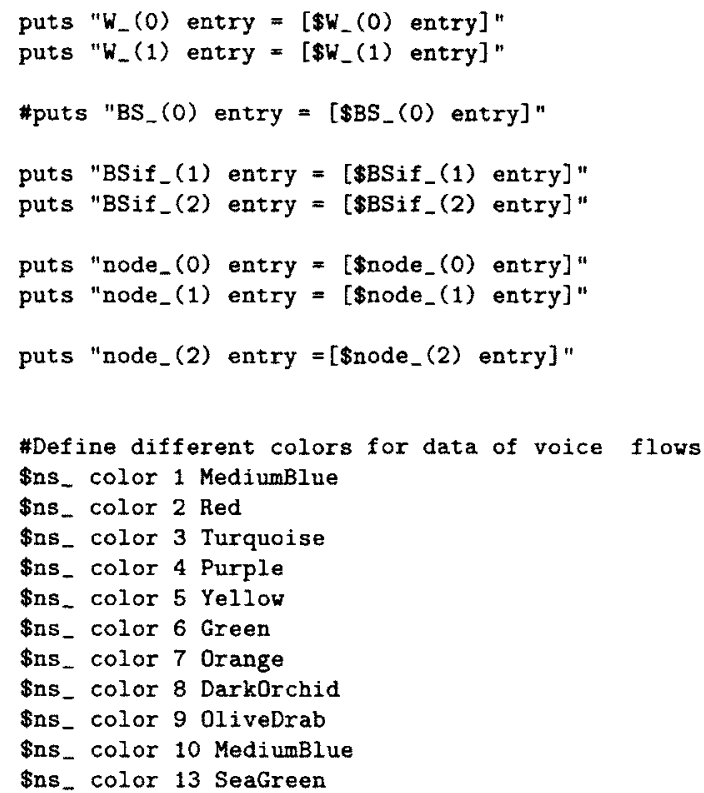

set em_ [new ErrorModel]

\$em_ unit pkt

$\$ \mathrm{Sm}_{\text {- set rate_ }} 0.02$

\$em_ ranvar [new RandomVariable/Uniform]

\#\$node_(2) interface-errormodel \$em_

\#\$node_(2) interface-errormodel [\$val(err)]

\#\$node interface-errormodel \$em.

\#create links between wired and BS nodes

$\$ n s_{-}$duplex-link $\$ W_{-}(0) \$ W_{-}(1) 5 \mathrm{Mb} 2 \mathrm{~ms}$ LTEQueue/ULAirqueue \$ns_duplex-link \$W_(1) \$BS_(O) 5Mb 2ms LTEQueue/ULAirqueue

\#Queue/DropTail set limit_ 100

\#Application/Traffic/CBR_PP set rate_ $40048 \mathrm{~Kb}$

$\# \$ n s_{-}$duplex-link $\$ W_{-}(0) \$ W_{-}(1)$.5Mb 2ms DropTail

$\#$ \#ns_duplex-1ink $\$ W_{-}(1) \$ B S_{-}(0)$.5Mb 2ms DropTail

\$ns_duplex-link-op $\$ W_{-}(0) \$ W_{-}(1)$ orient down

\$ns_ duplex-1ink-op $\$ W_{-}(1)$ \$BS_(0) orient left-down

\$ns_duplex-link \$BS_(0) \$BSif_(1) 5Mb 2ms LTEQueve/ULAirqueve \$ns_duplex-link \$BS_(O) \$BSif_(2) 5Mb 2ms LTEQueue/ULAirqueue

\$ns_duplex-link-op \$BS_(0) \$BSif_(1) orient wright-down \$ns_duplex-link-op \$BS_(0) \$BSif_(2) orient lef $t$-down

\#\$node_(1) base-station [AddrParams addr2id

[\$node_(0) node-addr]] 


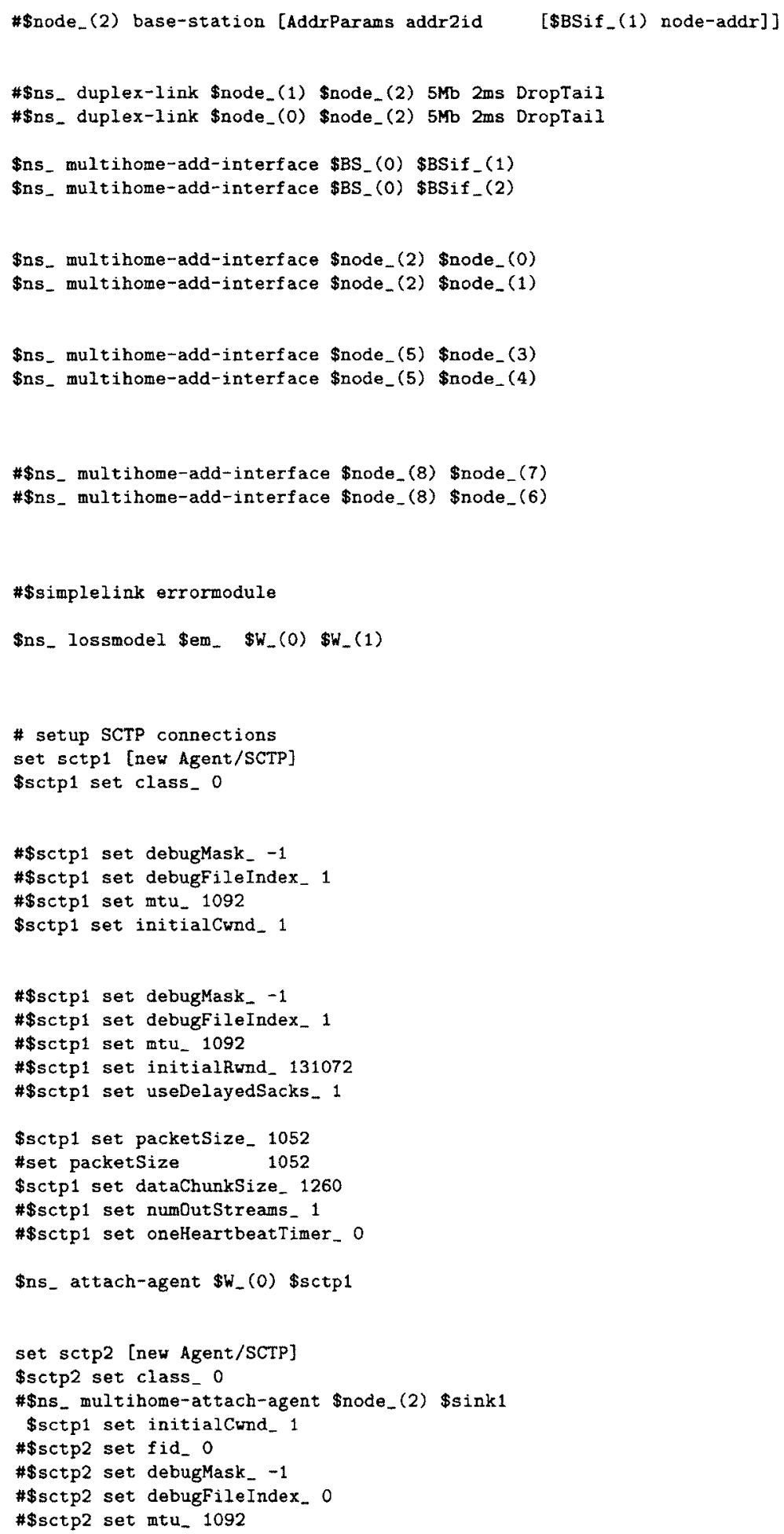


\$sctp2 set dataChunkSize 1260

\#\$sctp2 set numDutStreams 1

\#\$sctp2 set oneHeartbeatTimer_ 0

\$sctp2 set packetSize_ 1052

\#\$sctp2 set_filename st_a01

\$ns_attach-agent \$node_(2) \$sctp2

\$ns_ connect \$sctp2 \$sctp1

\#\$sctp1 set-primary-destination .0127

set tcp [new Agent/TCP]

\$tcp set class_ 2

set sink [new Agent/TCPSink]

\$ns - attach-agent \$node_(2) \$tcp

$\$ n s_{-}$attach-agent $\$ W_{-}(0)$ \$sink

\#\$ns_attach-agent \$node_(2) \$sink2

\$ns_ connect \$tcp \$sink

\#set ftp2 [new Application/FTP]

\#\$tp2 attach-agent \$tcp2

\#\$ns_at \$opt (ftp2-start) "\$ftp2 start"

\#\$sctp1 set-primary-destination _o127

set agent [new Agent/TCP]

\$ns_attach-agent \$node_(2) \$agent

\#\$agent set class_ 1

\$agent set packetSize_ 1400

\#\$agent set initialCwnd. 2

\#\$agent set cwnd_ 2

\#\$agent set class_ 1

*set size 500

\$agent set color 6

\$agent set class 2

\#\$agent set packetSize_ 2000

set sink [new Agent/TCPSink]

\$ns_attach-agent \$W_(0) \$sink

\$ns_ connect \$agent \$sink

set agent2 [new Agent/TCP]

\$ns _ attach-agent \$node_(8) \$agent2

\$agent2 set class 3

\$agent2 set packetSize_ 1400

\# \$agent set initialcwnd_ 2

\$agent2 set cwnd. 2

\#agent set class_ 1

\#set size 500

\$agent2 set color 1 
\#\$agent set packetSize_ 2000

set sink2 [new Agent/TCPSink]

\$ns_attach-agent \$W_(1) \$sink2

\$ns_ connect \$agent2 \$sink2

set agent1 [nev Agent/TCP]

\$as_attach-agent \$node_(5) \$agent 1

\$agent1 set class. 3

\$agent1 set color 4

set sink1 [new Agent/TCPSink]

\$ns_attach-agent \$W_(1) \$siak1

\$ns_ connect \$agent1 \$sink1

set app [new Application/FTP]

\#set app [new Application/Traffic/CBR]

set app [new Application/FTP]

set app1 [new Application/FTP]

set app2 [new Application/FTP]

\#\$app set rate $10000000 \mathrm{~kb}$

set realaudio(\$i) [new Application/Traffic/RealAudio]

\#set ftp1 [new Application/FTP]

\#\$ftp1 set rate $10000000 \mathrm{Mb}$

\$realaudio(\$i) attach-agent \$sctp2

\#\$video1 attach-agent \$sctp2

\#\$video1 attach-tracefile \$trace_file

\#\$realaudio(\$i) attach-agent \$agent

\#\$ns_at .06 "\$realaudio(\$i) start"

\#\$ns_at 3.6 "\$video1 start"

\#\$sctp2 force-source_o127

\#\$realaudio(\$i) attach-agent \$agent1

\$app2 attach-agent \$agent2

\$app1 attach-agent \$agent1

$\$ n s_{\text {_ at . }} 16$ "\$realaudio(\$i) start"

\#\$ns_ at 3.6 "\$app1 start"

\$ns_at 4.16 "\$app2 start"

$\$ n s_{-}$at 10.16 "\$app2 stop"

proc redirectTraffic \{\}\{

global multiFaceNode sctp ifaceo if ace1 ns routero router

\# sctp1 sctp2 node_(1) BSif_. $W_{-}$BS_

\#\$sctp_(1) set-primary-destination \$iface

$\#$ \#ns connect \$sctp_(1) \$sctp_(2) 


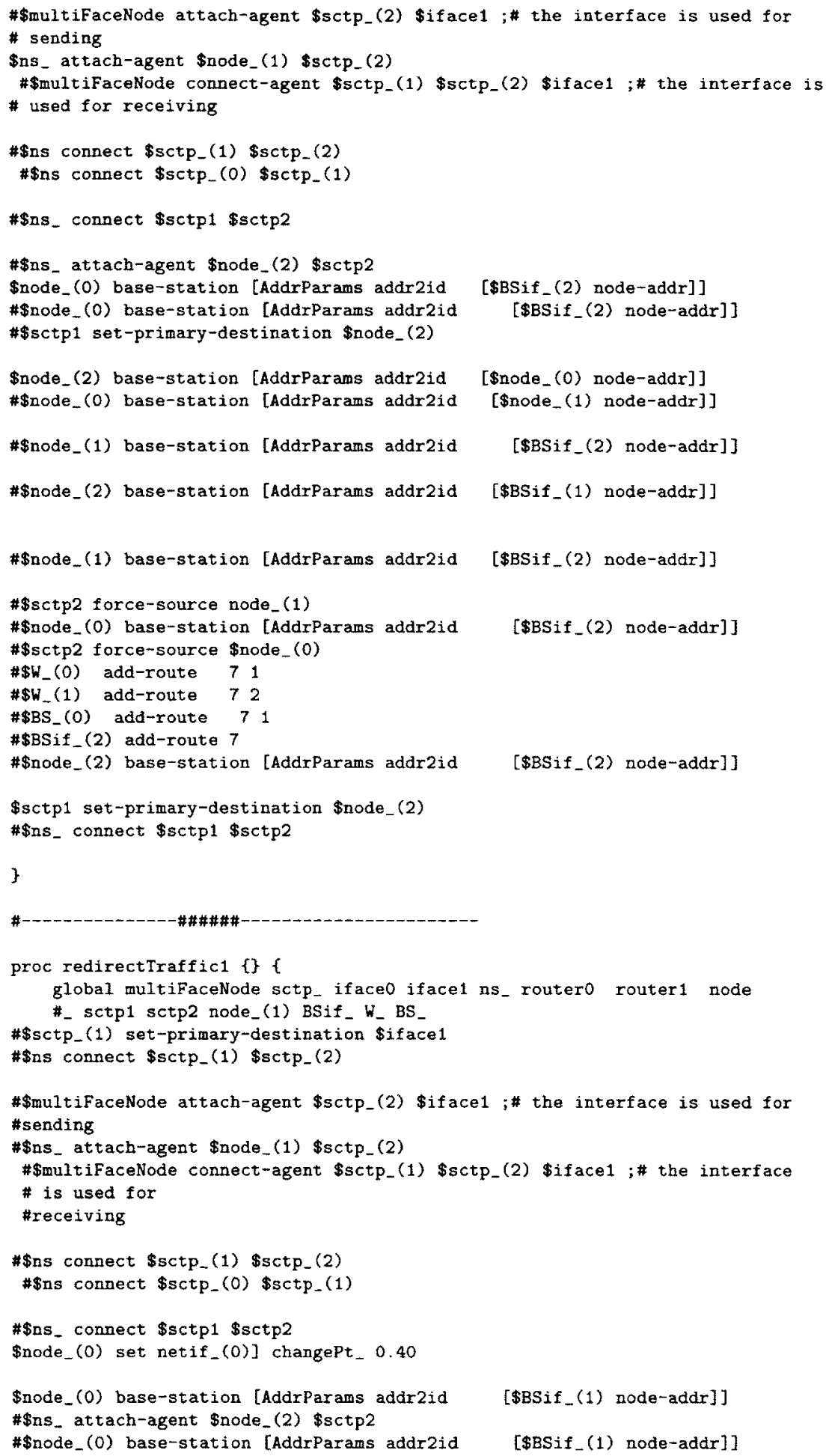


\#\$node_(1) base-station [AddrParams addr2id \$node_(2) base-station [AddrParams addr2id

\#\$node_(1) base-station [AddrParams addr2id

\#\$sctp2 force-source node_(1)

\#\$node_(0) base-station [AddrParams addr2id

\#\$sctp2 force-source \$node_(0)

\#\$W_(0) add-route 71

\#\$W_(1) add-route 72

\#\$BS_(0) add-route 71

\#\$BSif_(2) add-route 7

\#\$node_(2) base-station [AddrParams addr2id

\$sctp1 set-primary-destination \$node_(2)

\#\$n_ connect \$sctp1 \$sctp2

\}

proc redirectTraffic2 \{\}\{

global multifaceNode sctp_. ifaceo iface1 ns. Foutero router1 node.

\#sctp1 sctp2 node_(1) BSif $W_{-}$BS

\#\$sctp_(1) set-primary-destination \$iface1

\#\$s connect $\$$ sctp_(1) \$sctp_(2)

\#\$multifaceNode attach-agent \$sctp_(2) \$iface 1 ; the interface is used for \# sending

\#\$ns_attach-agent \$node_(1) \$sctp_(2)

\#\$multifaceNode connect-agent \$sctp_(1) \$sctp_(2) \$iface1; \# the interface

\# is used for

\# receiving

\#\$s connect \$sctp_(1) \$sctp_(2)

\# \$ns connect $\$ s c t p_{-}(0) \$ s c t p_{-}(1)$

$\#$ \#n__ connect \$sctp1 \$sctp2

\#\$ns_attach-agent \$node_(2) \$sctp2

\#\$node_(1) base-station [AddrParams addr2id

\#\$node_( 0 ) base-station [AddrParams addr2id

\#\$sctp1 set-primary-destination \$node_(2)

\#\$node_(2) base-station [AddrParams addr2id

\$node_(0) base-station [Addrparams addr2id

\$node_(2) base-station [AddrParams addr2id

\#\$node_(1) base-station [AddrParams addr2id

\#\$sctp2 force-source node_(1)

\#\$node_(0) base-station [AddrParams addr2id

\#\$sctp2 force-source \$node_(0)

\$W_(0) add-route 71

$\$ W_{-}(1)$ add-route 72

\#\$BS_(0) add-route 71

\#BSif_(2) add-route 7

\#\$node_(2) base-station [AddrParams addr2id

[\$BSif_(2) node-addr]]

[\$BSif_(2) node-addr]]

[\$BSif_(1) node-addr]]

[\$node_(1) node-addr]

[\$BSif_(2) node-addr]]

[\$node_(1) node-addr] ]

[\$BSif_(2) node-addr]]

[\$BSif_(2) node-addr]]

\$sctp1 set-primary-destination \$node_(2)

\#\$ns_connect \$sctp1 \$sctp2

\}

$\#$ \#ns_ at 1.0 "redirectTraffic" 


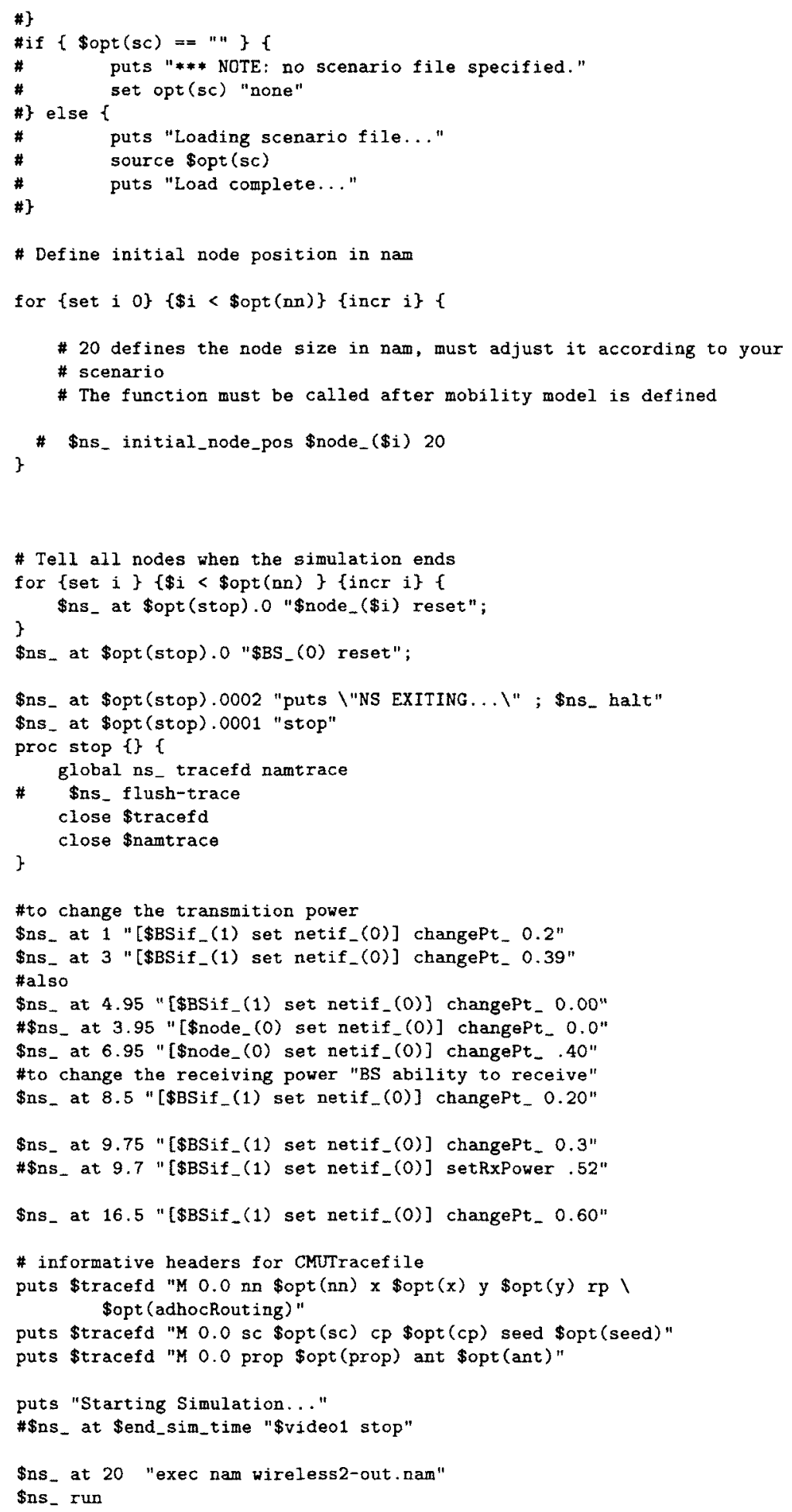




\section{A.2 Video Traffic via TCP and SCTP}

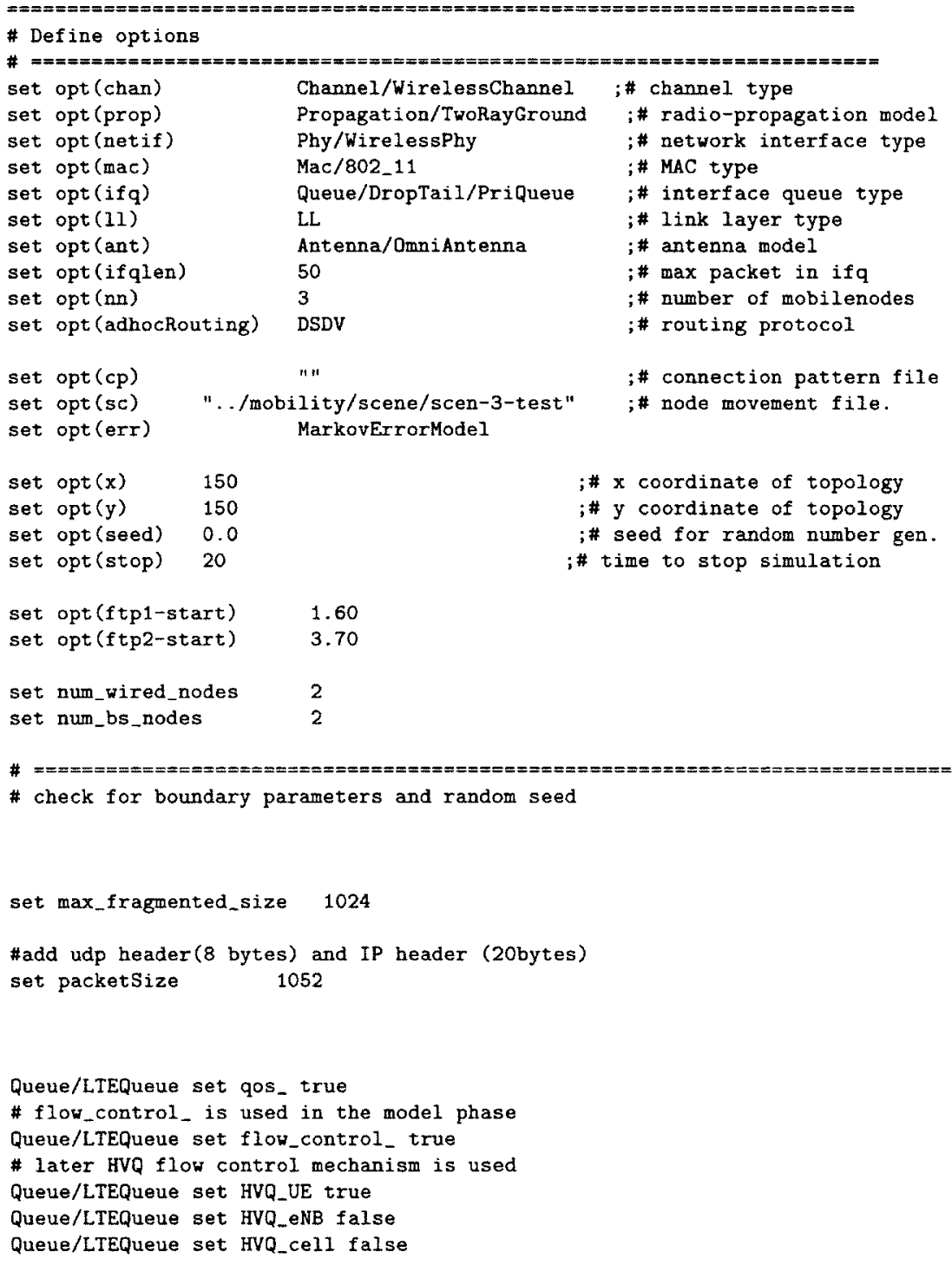




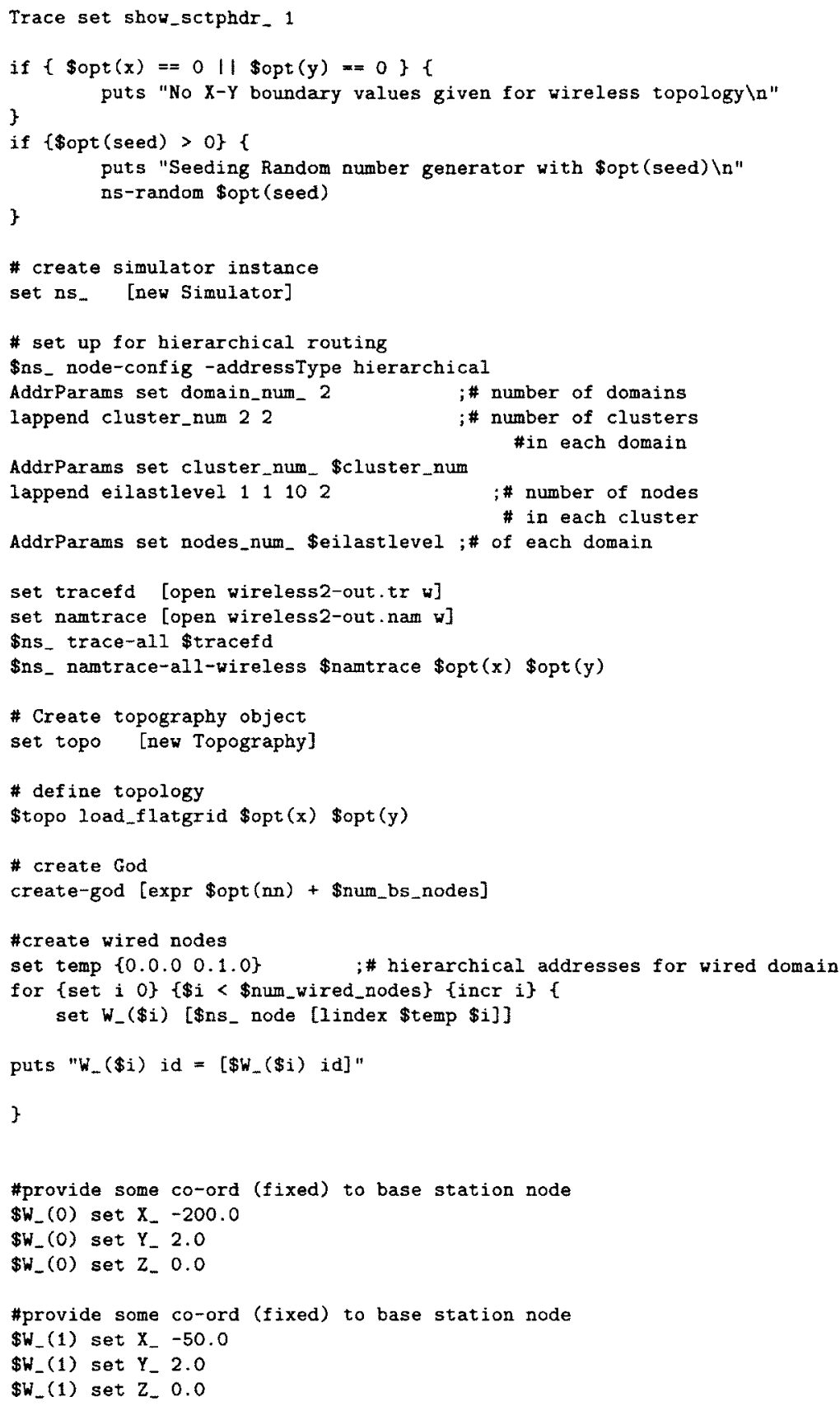

set chan_1 [new \$opt (chan)]

set chan 2 [new \$opt (chan)]

set chan 3 [new \$opt (chan)] 


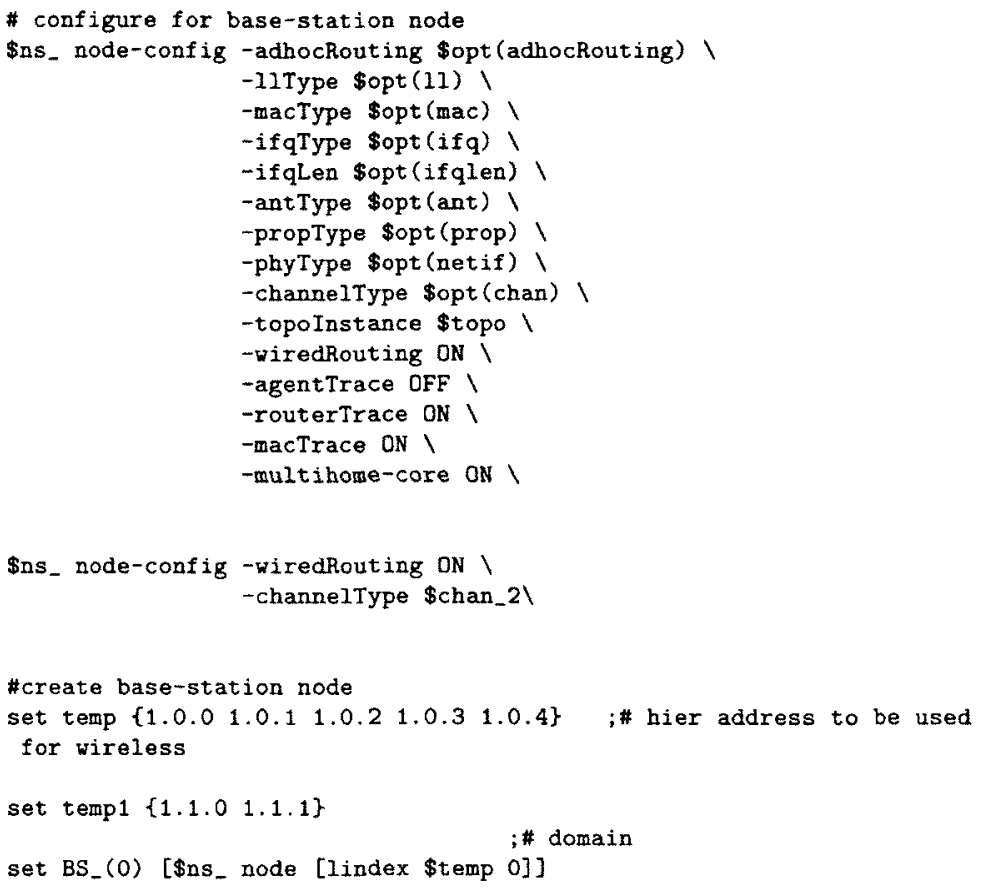


$\$ B$ Sif $_{-}(2)$ set $X_{-} 0.0$

\$BSif_(2) set $Y_{-} 48.0$

\$BSif_(2) set $z_{-} 0.0$

puts "BSif_(2) id $=\left[\$ B S i f_{-}(2)\right.$ id $] "$

\$BSif_(2) label "BSif_(2)"

*set BSif1 [\$ns_ node]

\#set BSif2 [\$ns_ node]

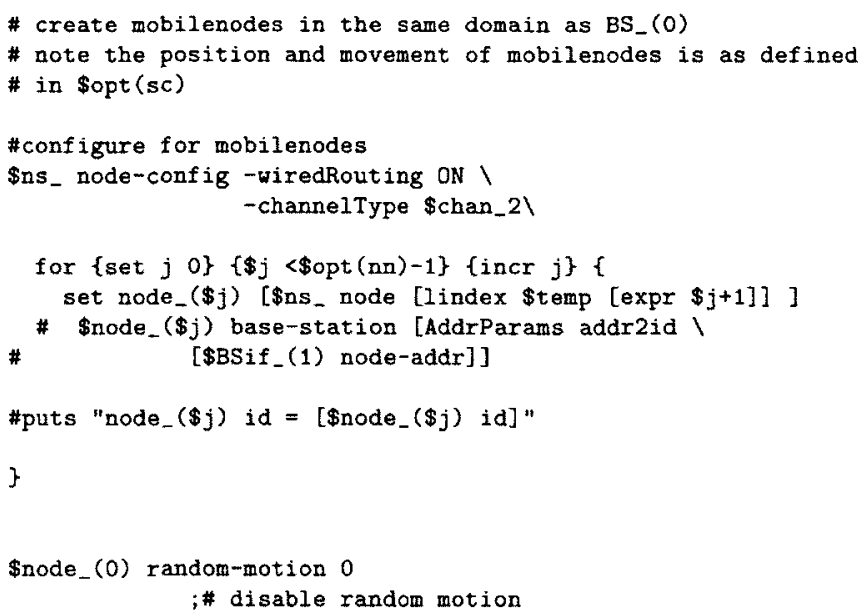




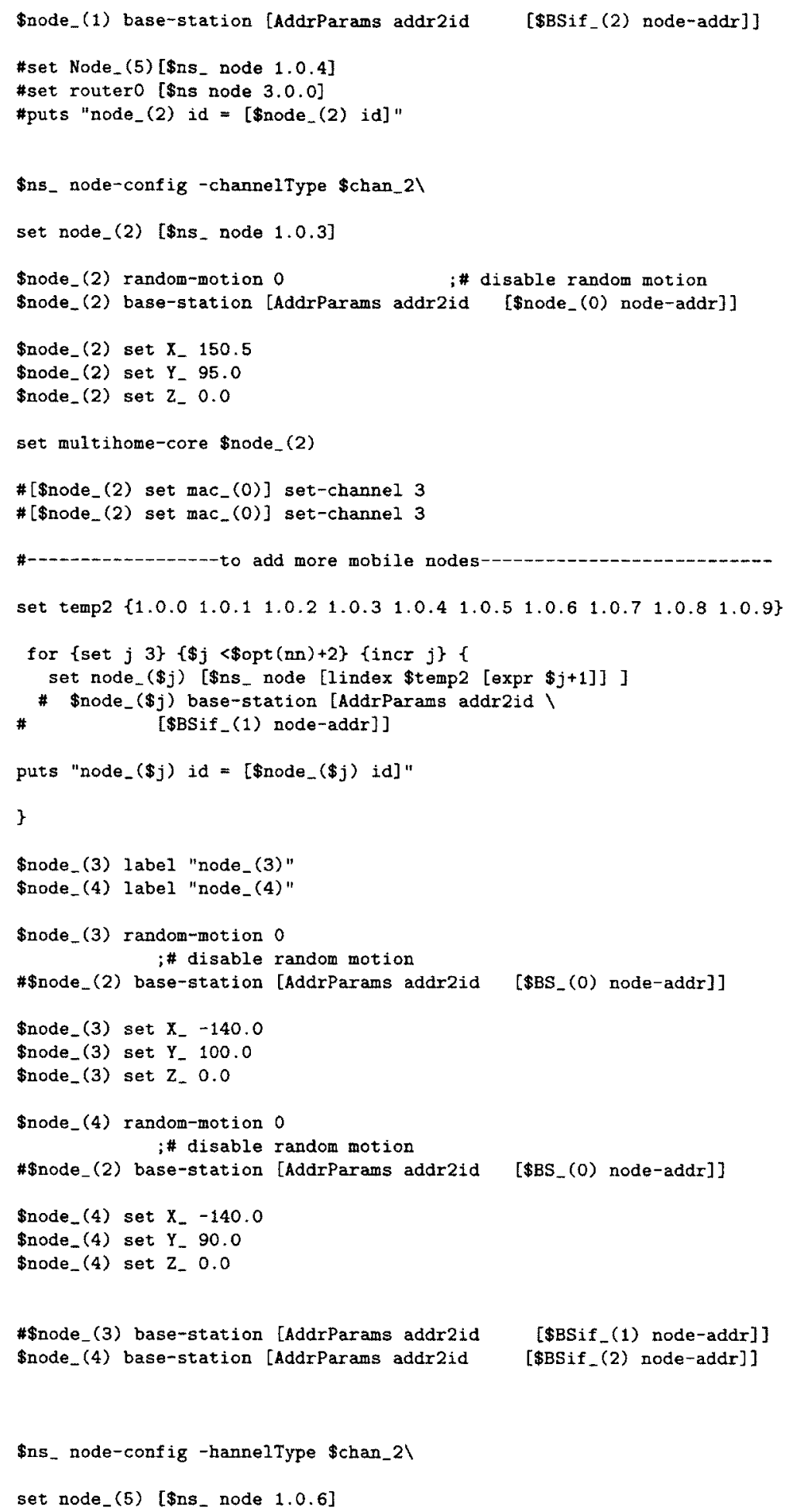

\$ns_ node-config -hannelType \$chan_2\

set node_(5) [\$ns_ node 1.0 .6$]$ 


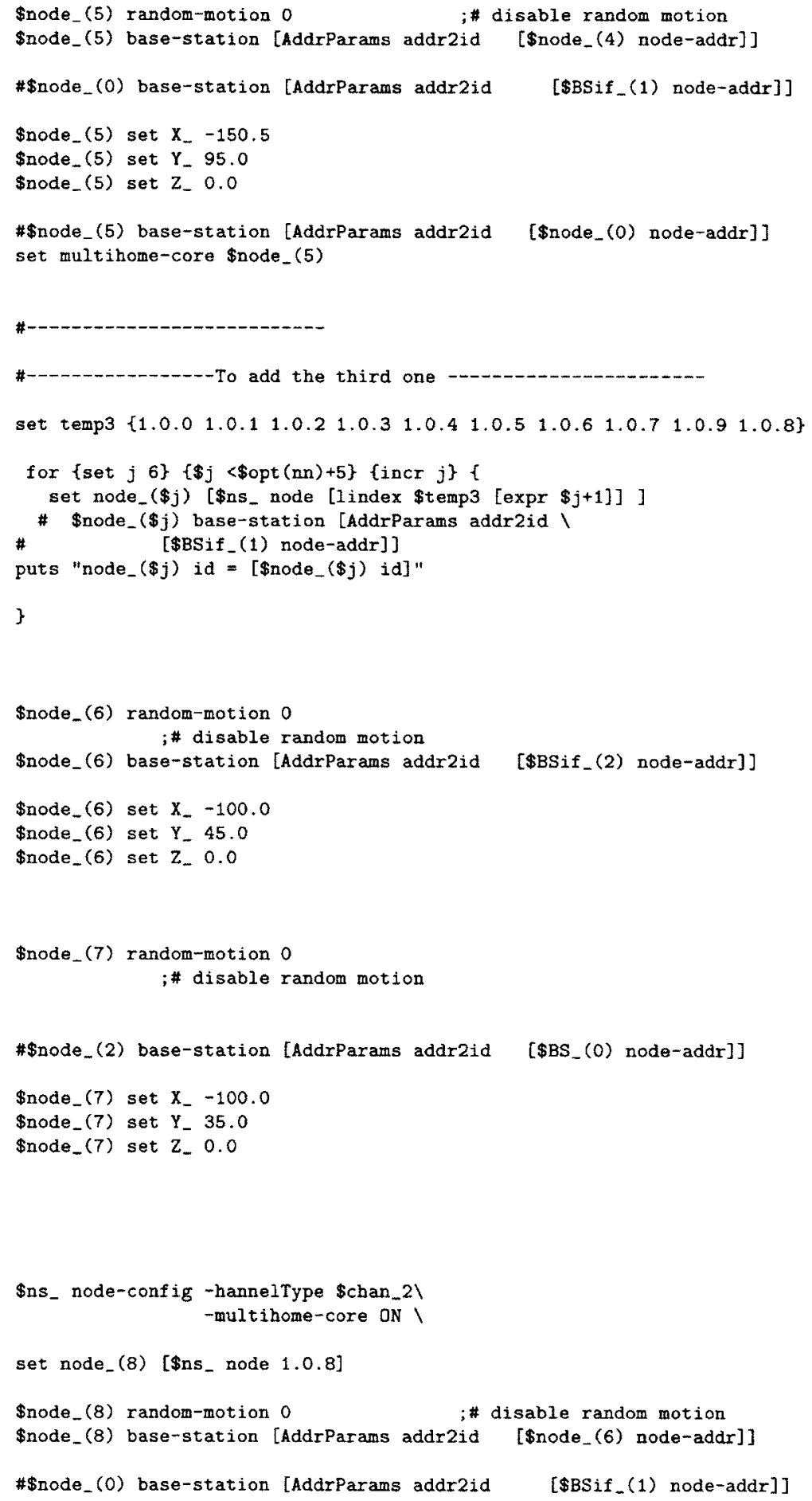


$\$$ node_(8) set $X_{-}-110.0$

\$node_(8) set $Y_{-} 40.0$

\$node_(8) set $Z_{\ldots} 0.0$

\#\$node_(8) base-station [AddrParams addr2id

[\$node_(0) node-addr]]

set multihome-core \$node_( 8 )

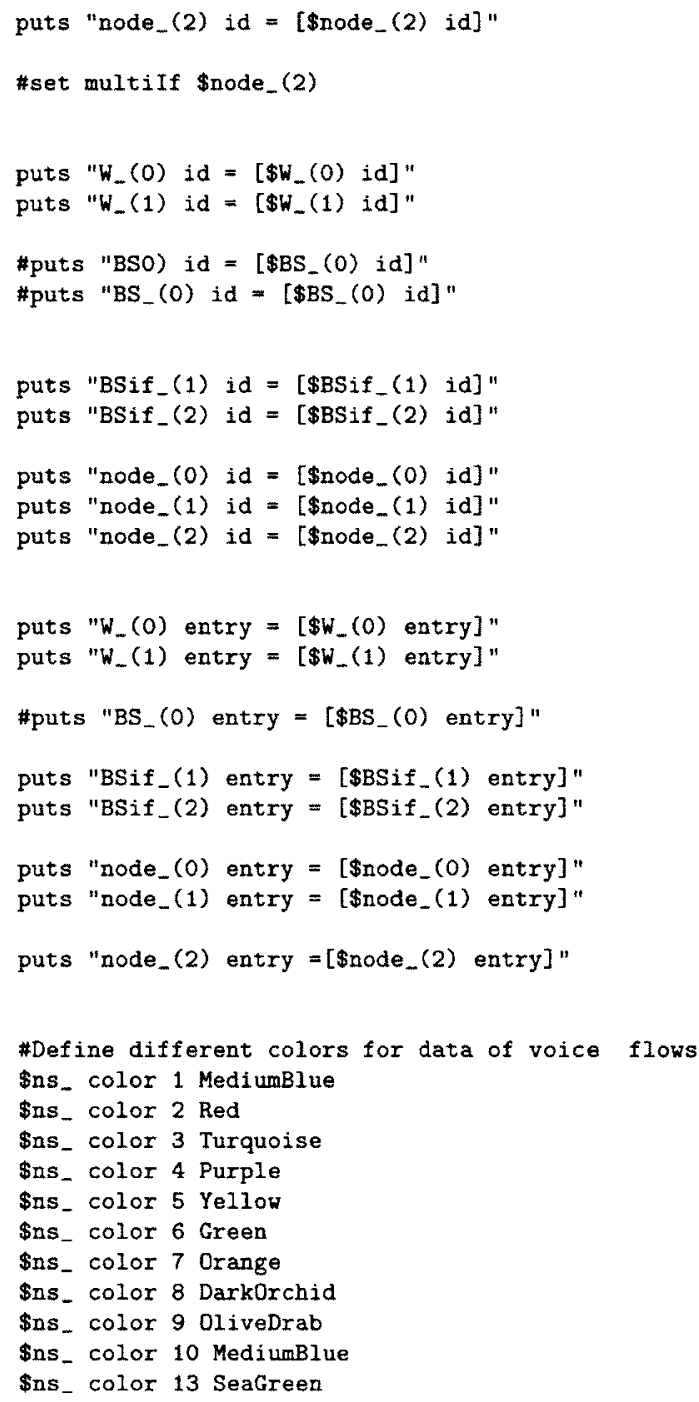

set em_ [new ErrorModel] 
\$em_ unit pkt

Sem_ set rate 0.02

Sem_ranvar [new RandomVariable/Uniform]

\#\$node_(2) interface-errormodel \$em.

\#\$node_(2) interface-errormodel [\$val (err)]

\#\$node interface-errormodel \$em_

\#create links between wired and BS nodes

\$ns_duplex-link \$W_(0) $\$ W_{-}$(1) 5Mb 2ms LTEQueue/ULAirqueue

\$ns_duplex-link \$W_(1) \$BS_(0) 5Mb 2ms LTEQueue/ULAirqueue

\#Queue/DropTail set limit_ 100

\#Application/Traffic/CBR_PP set rate_ $40048 \mathrm{~Kb}$

\#\$ns_duplex-link $\$ W_{-}(0) \$ W_{-}(1)$.5Mb 2ms DropTail

\#\$ns_duplex-link \$W_(1) \$BS_(0) .5Mb 2ms DropTail

\$ns_ duplex-link-op $\$ W_{-}(0) \$ W_{-}(1)$ orient down

\$ns_duplex-link-op $\$ W_{-}(1)$ BES_(0) orient left-down.

$\$ n s_{-}$duplex-link \$BS_(0) \$BSif_(1) 5Mb 20ms DropTai]

\$ns_duplex-1ink \$BS_(0) \$BSif_(2) 5Mb 20ms DropTail

\$ns duplex-link-op \$BS_(0) \$BSif_(1) orient wright-down

\$ns_ duplex-link-op \$BS_(0) \$BSif_(2) orient left-down

\#\$node_(1) base-station [AddrParams addr2id

[\$node_(0) node-addr]]

\#\$node_(2) base-station [AddrParams addr2id

[\$BSif_(1) node-addr]]

\#\$ns_duplex-1ink \$node_(1) \$node_(2) $5 \mathrm{Mb} 2 \mathrm{~ms}$ DropTail

\#\$ns_duplex-1ink \$node_(0) \$node_(2) 5Mb 2ms DropTail

\$ns_ multihome-add-interface \$BS_(0) \$BSif_(1)

\$ns_ multihome-add-interface $\$ B S_{-}(0)$ \$BSif_(2)

\$ns_ multihome-add-interface \$node_(2) \$node_(0)

$\$ n s_{-}$multihome-add-interface \$node_(2) \$node_(1)

\$ns_ multihome-add-interface \$node_(5) \$node_(3)

Sns_multihome-add-interface \$node_(5) \$node_(4)

\#\$ns_ multihome-add-interface \$node_(8) \$node_(7)

\#\$s_ multihome-add-interface \$node_(8) \$node_(6)

\#\$simplelink errormodule

$\$ n s_{-}$lossmodel $\$ e m_{-} \$ W_{-}(0) \$ W_{-}(1)$

\#---to include the framed video trafic---

set original_file name st_a. 01 


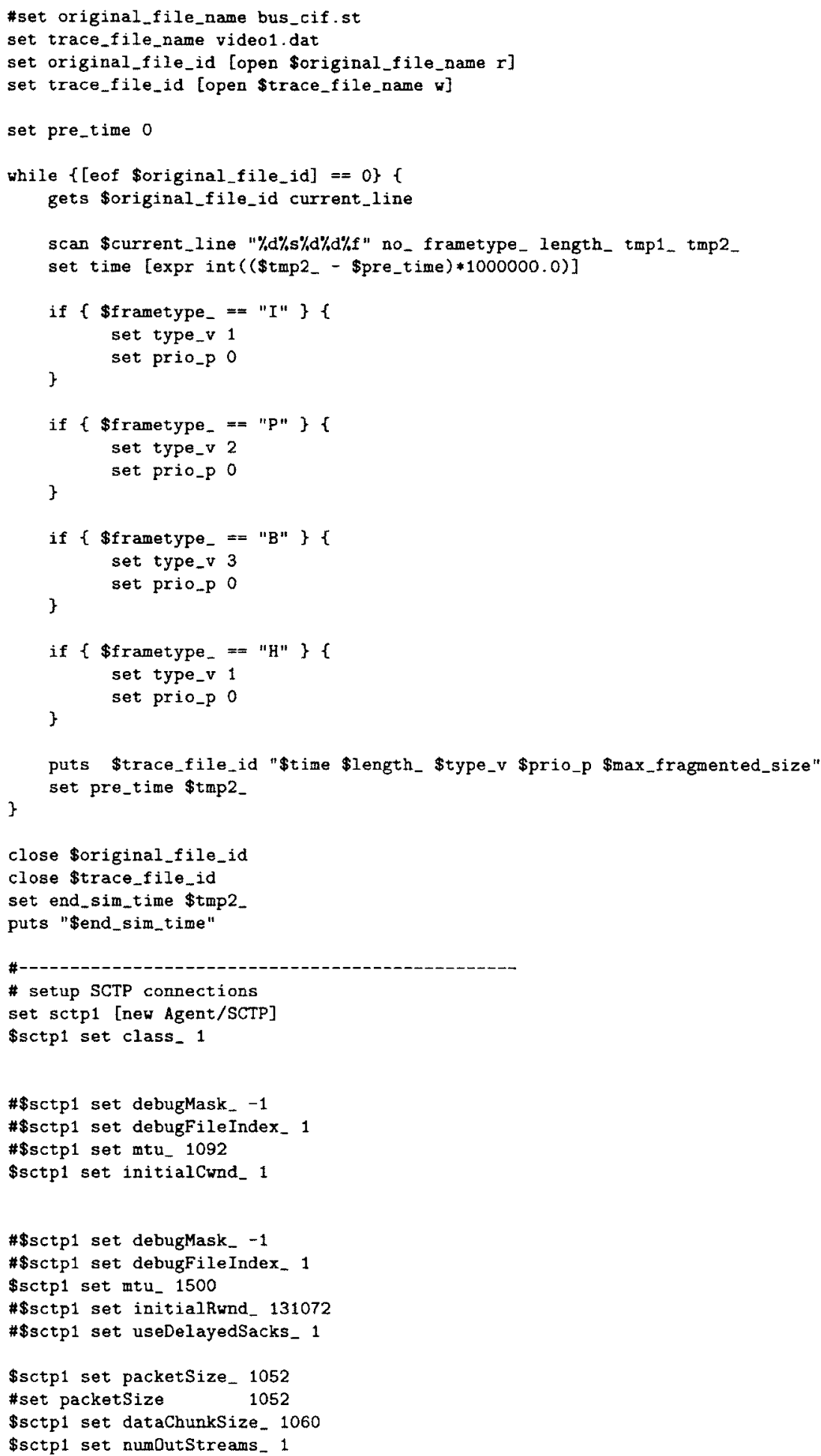


\#sctp1 set oneHeartbeatTimer_ 0

\$ns_attach-agent $\$ w_{-}(0) \$ s c t p 1$

set sctp2 [new Agent/SCTP]

$\$ s c t p 2$ set class_ 1

\#\$ns_ multihome-attach-agent \$node_(2) \$sink1

\#\$sctp2 set fid_ 0

\#\$sctp2 set debugMask -1

\#\$sctp2 set debugFileIndex 0

\$sctp2 set mtu_ 1092

\$sctp2 set dataChuriksize 1060

$\# \$$ sctp2 set numOutStreams_ 1

\#\$sctp2 set oneHeartbeatTimer 0

\$sctp2 set packetSize. 1052

\#\$sctp2 set_filename st_a01

\$ns_attach-agent \$node_(2) \$sctp2

\$ns_ connect \$sctp2 \$sctp1

\#\$sctp1 set-primary-destination _0127

set tcp [new Agent/TCP]

$\$$ tcp set class_ 3

set sink [new Agent/TCPSink]

$\$ n s_{-}$attach-agent \$node_(2) \$tcp

$\$ n s_{\text {_ attach-agent } \$ \mathrm{~W}_{-}(0) \$ \text { sink }}$

\#\$ns_attach-agent \$node_(2) \$sink2

\$ns_ connect \$tcp \$sink

\#set ftp2 [new Application/FTP]

\#\$fp2 attach-agent \$tcp2

\#\$ns_at \$opt(ftp2-start) "\$ftp2 start"

\#\$sctp1 set-primary-destination _0127

set agent [new Agent/TCP]

\$ns_attach-agent \$node_(2) \$agent

\$agent set class 1

\#\$agent set packetSize_ 1400

\#\$agent set initialCwnd. 2

\# \$agent set cwnd_ 2

Sagent set class 1

\#set size 500

\$agent set color 6

\$agent set class. 3

\#\$agent set packetSize_ 2000

set sink [new Agent/TCPSink]

\$ns_attach-agent $\$ w_{-}(0)$ \$sink

\$ns_ connect \$agent \$sink 
set agent 2 [new Agent/TCP]

\$ns_attach-agent \$node_(8) \$agent2

\$agent2 set class_ 3

\#\$agent2 set packetSize_ 1400

\#\$agent set initialCwnd_ 2

\$agent 2 set cwnd_ 2

Sagent set class_ 1

\#set size

500

Sagent2 set color 4

\#\$agent set packetSize_ 2000

set sink2 [new Agent/TCPSink]

$\$ n s_{-}$attach-agent $\$ W_{-}(1)$ \$sink2

\$ns_ connect \$agent2 \$sink2

set agent1 [new Agent/TCP]

\$ns_attach-agent \$node_(5) \$agent 1

\$agent1 set class_ 3

\$agent2 set color 4

set sink1 [new Agent/TCPSink]

\$ns_attach-agent \$W_(1) \$sink1

\$ns_ connect \$agent1 \$sink1

set trace file [new Tracefile]

\$trace_file filename \$trace_file_name

set video1 [new Application/Traffic/myEvalvid]

set app [new Application/FTP]

\# set app [new Application/Traffic/CBR]

set app [new Application/FTP]

set app1 [new Application/FTP]

set app2 [new Application/FTP]

\#\$app set rate $10000000 \mathrm{~kb}$

set realaudio(\$i) [new Application/Traffic/RealAudio]

\#set ftp1 [new Application/FTP]

\#ftp1 set rate $10000000 \mathrm{Mb}$

\# \$realaudio(\$i) attach-agent \$sctp2

\$video1 attach-agent \$sctp2

Svideo1 attach-tracefile \$trace file

\$realaudio $(\$ i)$ attach-agent \$agent

\#\$ns_at .06 "\$realaudio(\$i) start"

\$ns_at 0.06 "\$video1 start"

\#\$sctp2 force-source _0127 


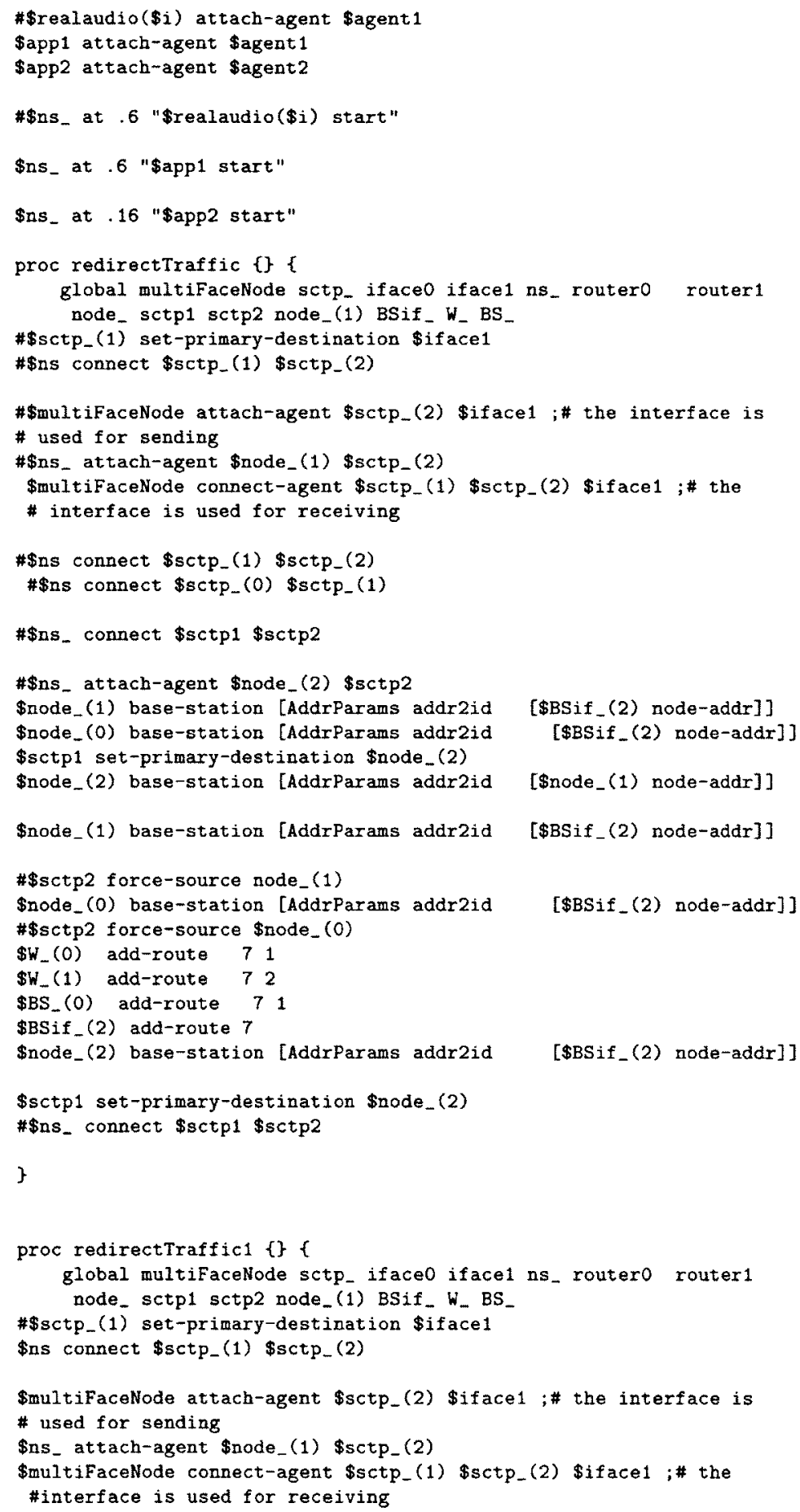


\#\$ns confect \$sctp_(1) \$sctp_(2)

$\#$ \$ns connect $\$$ sctp_(0) \$sctp_(1)

$\$ n s_{-}$connect $\$$ sctp1 \$sctp2

\$node_( $O$ ) base-station [AddrParams addr2id

\#\$ns_attach-agent \$node_(2) \$sctp2

Snode_(0) base-station [AddrParams addr2id

\$node_(1) base-station [AddrParams addr2id

[\$BSif_(1) node-addr]]

\$node_(2) base-station [AddrParams addr2id

[\$BSif_(1) node-addr]]

[\$BSif_(2) node-addr]]

\$node_(1) base-station [AddrParams addr2id

[\$BSif_(2) node-addr]]

\#\$sctp2 force-source node_(1)

\$node_( 0 ) base-station [AddrParams addr2id

[\$BSif_(2) node-addr]]

\#\$sctp2 force-source \$node_( 0$)$

$\$ W_{-}(0)$ add-route 71

$\$ W_{-}$(1) add-route 72

\$BS_(0) add-route 71

\$ESif_(2) add-route 7

\$node_(2) base-station [AddrParams addr2id [\$BSif_(2) node-addr]]

\$sctp1 set-primary-destination \$node_(2)

\#\$s. connect \$sctp1 \$sctp2

\}

proc redirectTraffic 3\{\}

global multifaceNode sctp_ ifaceo iface1 ns _ routero router1 node sctp1 sctp2 node_(1) BSif_. W BS_

$\$ s c t p_{-}(1)$ set-primary-destination \$iface1

$\#$ \#s connect \$sctp_(1) \$sctp_(2)

\#\$multiFaceNode attach-agent \$sctp_(2) \$iface1; \# the interface is used for \# sending

\#\$ns_attach-agent \$node_(1) \$sctp_(2)

\$multiFaceNode connect-agent \$sctp_(1) \$sctp_(2) \$iface1;

\# is used for receiving

\# \$ns connect $\$$ sctp_( 1$) \$ s c t p_{-}(2)$

$\#$ \#s connect $\$ s c t p_{-}(0) \$ s c t p_{-}(1)$

$\#$ \$ns_ connect $\$$ sctp1 $\$$ sctp2

\#ns_attach-agent $\$$ node_(2) \$sctp2

\$node_(1) base-station [AddrParams addr2id

\$node_(0) base-station [AddrParams addr2id

\$sctp1 set-primary-destination \$node_(2)

\$node_(2) base-station [AddrParams addr2id

\$node_(1) base-station [AddrParams addr2id

[\$BSif_(2) node-addr]]

[\$BSif_(2) node-addr]]

\#\$sctp2 force-source node_(1)

\$node_(0) base-station [AddrParams addr2id

\$sctp2 force-source \$node_(0)

$\$ W_{-}(0)$ add-route 71

$\$ W_{-}(1)$ add-route 72

\$BS_(0) add-route 71

\$BSif_(2) add-route 7

\$node_(2) base-station [AddrParams addr2id

[\$node_(1) node-addr]]

[\$BSif_(2) node-addr]]

[\$BSif_(2) node-addr]]

\$sctp1 set-primary-destination \$node_(2)

\#\$ns_ connect \$sctp1 \$sctp2 
proc redirectTraffics \{\}\{

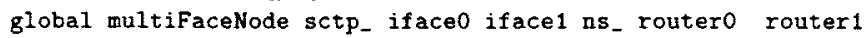

sctp1 sctp2 node_(1) BSif - $W_{-}$BS

\$sctp_(1) set-primary-destination \$iface1

\#\$n connect $\$$ sctp_(1) \$sctp_(2)

\#\$multifaceNode attach-agent $\$ s c t p_{-}(2)$ \$iface1; \# the interface is used for \# sending

\#\$ns_attach-agent \$node_(1) \$sctp_(2)

\$multifaceNode connect-agent \$sctp_(1) \$sctp_(2) \$iface1; \# the interface

\# is used for receiving

$\#$ \#ns connect $\$$ sctp_(1) \$sctp_(2)

$\#$ \#ns connect $\$$ sctp_(0) $\$ s c t p_{-}(1)$

\#\$s_connect \$sctp1 \$sctp2

\$node_(0) base-station [AddrParams addr2id [\$BSif_(1) node-addr]]

\#\$ns_attach-agent \$node_(2) \$sctp2

\$node_(0) base-station [AddrParams addr2id

\$node_(1) base-station [AdarParams addr2id

[\$BSif_(1) node-addr]]

\$node_(2) base-station [AddrParams addr2id

$\left[\$ B S i f_{-}(2)\right.$ node-addr]]

\#\$ode_(1) base-station [AddrParams addr2id

[\$node_(0) node-addr]]

\#\$sctp2 force-source node_(1)

\#\$node_( 0 ) base-station [AddrParams addr2id

\#\$sctp2 force-source \$node_( 0$)$

$\$ W_{-}(0)$ add-route 71

SW_(1) add-route 72

\$BS_(0) add-route 71

\$BSif_(2) add-route 7

\$node_(2) base-station [AddrParams addr2id

[\$BSif_(2) node-addr]]

\$sctp1 set-primary-destination \$node_(2)

\#\$ns_ connect \$sctp1 \$sctp2

\}

$\$ n s_{-}$at 3.0660 "redirectTraffic3"

$\$ n s_{\text {_ at }} 3.0900$ "redirectTrafic4"

\$ns_ rtmodel-at 5.29 down $\$ B S_{-}(0)$ BSif_(2)

\$ns r rmodel-at 1.0 down $\$ B S i f_{-}(2)$ \$BS_(0)

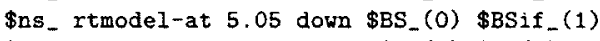

$\$ n s_{-}$rtmodel-at 4.950 down $\$ W_{-}(0) \$ W_{-}(1)$

$\$ n s_{-}$rtmodel-at 5.3 up $\$ B S_{-}(0)$ \$BSif_(2)

$\$ n s_{-}$rtmodel-at 2.4 up $\$ B S_{-}(0) \$ W_{-}(1)$

\$ns r rmodel-at 2.4 up \$BSif_(2) \$BS_(0)

\$ns_ rtmodel-at 8.95 up $\$ B S_{-}(0)$ \$BSif_(1)

$\$ n s_{-}$rtmodel-at 5.0 up $\$ W_{-}(0) \$ W_{-}(1)$

$\$ n s_{-}$rtmodel-at 8.850 down $\$ W_{-}(0) \$ W_{-}(1)$

$\# \$ n s_{\text {_ }}$ rtmodel-at 9.3 up $\$ W_{-}(0) \$ W_{-}(1)$

\#\$ns_ at 4.980 "redirectTraffic"

Ins_at 7.98 "redirectTraffict"

\#\$ns_at 2.38 "redirectTraf $f i c "$

\# MH starts to move towards FA $(640,610)$ at a speed of $20 \mathrm{~m} / \mathrm{s}$ 
\#\$ns_ at 1.000000000000"\$node_(1) setdest 40.00000000000010 .000000000000 $20.000000000000 "$

\$ns at 1.000000000000 "\$node_(1) setdest 40.00000000000010 .000000000000 $90.000000000000^{\prime \prime}$

\$ns at 1.000000000000 "\$node_(0) setdest 35.00000000000020 .000000000000 $90.000000000000^{\prime \prime}$

\$ns_at 1.000000000000 "\$node_(2) setdest 37.5.000000000000 15.000000000000 $90.000000000000 "$

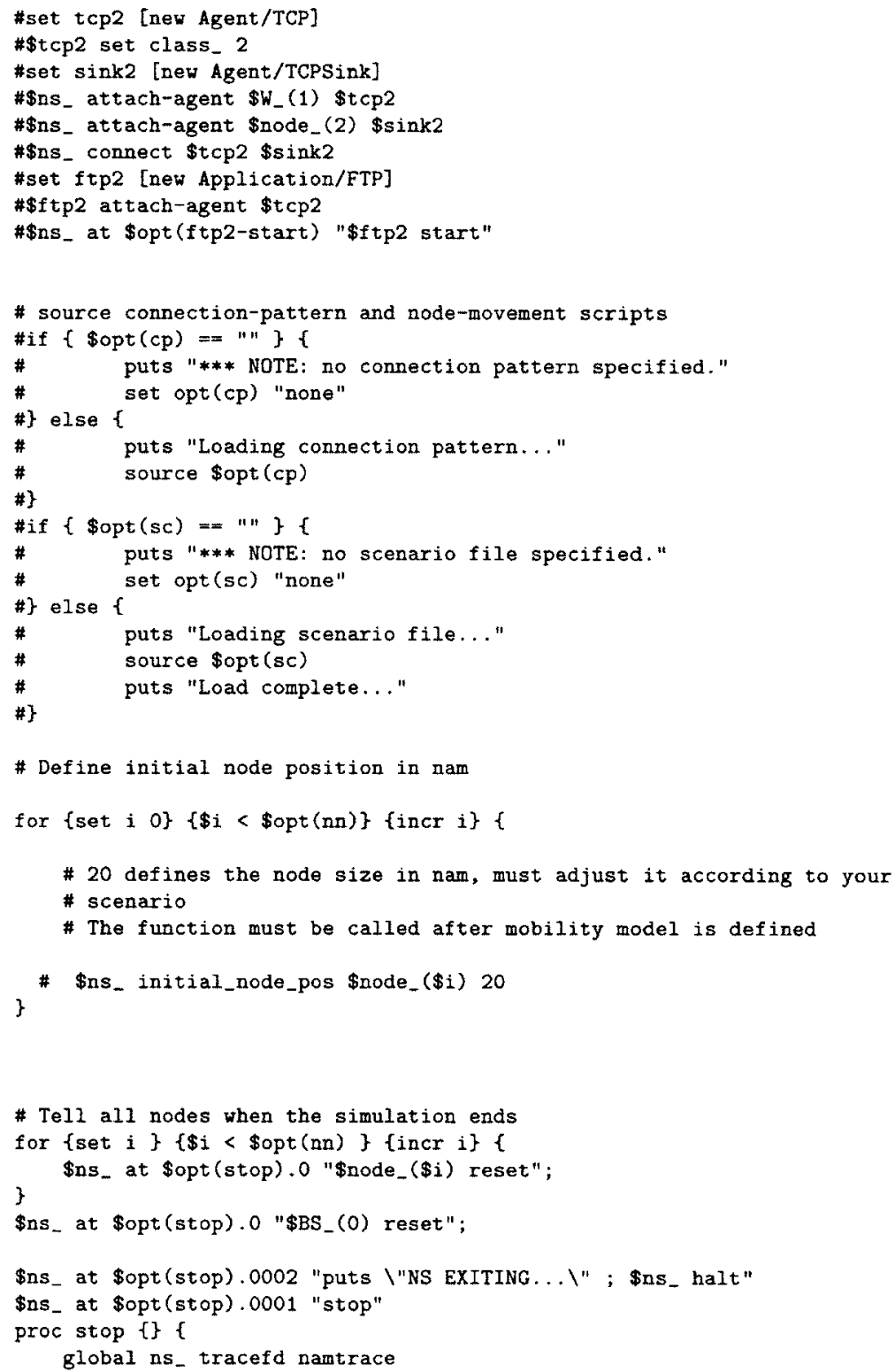




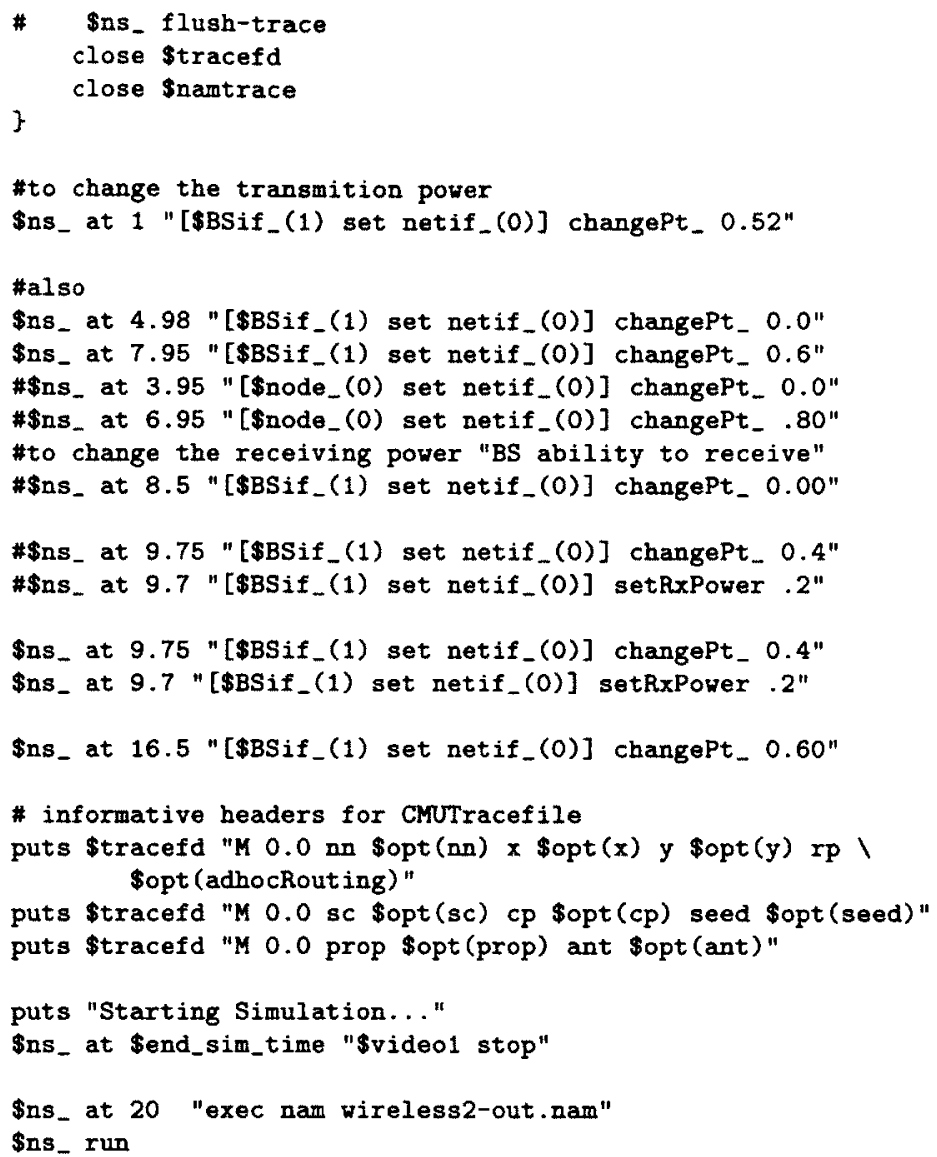

